\title{
Towards Chromanes by de novo Construction of the Benzene
}

\section{Ring}

Egor Geist ${ }^{a}$, Helge Berneaud-Kötz ${ }^{a}$, Tomas Baikstis ${ }^{b}$, Gerald Dräger $^{\mathrm{a}}$ and Andreas Kirschning ${ }^{\mathrm{a} *}$

a Institute of Organic Chemistry and Center of Biomolecular Drug Research (BMWZ) at Leibniz Universität Hannover,

Schneiderberg 1B, 30167 Hannover, Germany

b Sygnature Discovery, Biocity, Pennyfoot street, Nottingham NG11GR, United Kingdom

\section{Supporting Information}

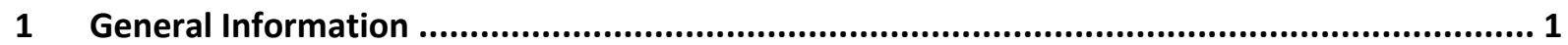

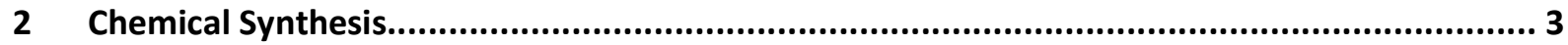

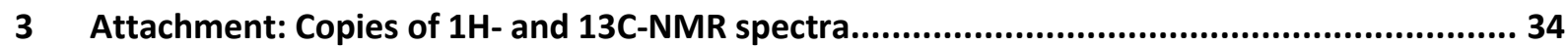

\section{General Information}

${ }^{1} \mathrm{H}$ NMR spectra were recorded with a Bruker Avance-400 (400 MHz) with DPC console and Bruker DRX-500 (500 MHz) with DRX-console at room temperature. Multiplicities are described with the following abbreviations: $\mathrm{s}$ - singlet, $\mathrm{d}$ - doublet, $\mathrm{t}$ - triplet, $\mathrm{q}$ - quartet, $\mathrm{m}$ - multiplet, $\mathrm{b}$ - broad. ${ }^{13} \mathrm{C}$ NMR spectra were recorded at $101 \mathrm{MHz}$ with a Bruker Avance-400 and at $125 \mathrm{MHz}$ with a Bruker DRX-500. Chemical shifts of ${ }^{1} \mathrm{H}$ - and ${ }^{13} \mathrm{C}-\mathrm{NMR}$ spectra are reported in $\delta(\mathrm{ppm})$. Multiplicities are described with the following abbreviations: $s$ - singlet (due to quaternary carbon), $d-$ doublet (methine), $\mathrm{t}$ - triplet (methylene), $\mathrm{q}$ - quartet (methyl), $\mathrm{m}$ - multiplet, $\mathrm{b}$ - broad. All coupling constants $J$ are expressed in Hertz. Supporting ${ }^{1} \mathrm{H}^{1} \mathrm{H}$ correlation (COSY) and ${ }^{1} \mathrm{H}-{ }^{13} \mathrm{C}$ correlation (HSQC, $\mathrm{HMBC}$ ) experiments were performed, if required.

Mass spectra were recorded with a type LCT (ESI) equipped with a lockspray dual ion source in combination with a WATERS Alliance 2695 LC system, or with a type QTOF premier (MICROMASS) spectrometer (ESI mode) in combination with a Waters Acquity S2 UPLC system equipped with a 
Waters Acquity UPLC BEH C18 $1.7 \mu \mathrm{m}$ (SN 01473711315545) column (solvent A: water + 0.1\% (v/v) formic acid, solvent $\mathrm{B}$ : $\mathrm{MeOH}+0.1 \%(\mathrm{v} / \mathrm{v})$ formic acid; flow rate $=0.4 \mathrm{~mL} / \mathrm{min}$; gradient (t [min]/solvent B [\%]): 0/5; 2.5/95; 6.5/95; 6.6/5; 8/5). Ion mass signals (m/z) are reported as values in atomic mass units. Retention times $\left(t_{\mathrm{R}}\right)$ are given in the experimental part.

High pressure reactions were conducted in a Hofer HP14 hydraulic press. A sealable teflon cyclinder was used as the reaction vessel (Figure 1).

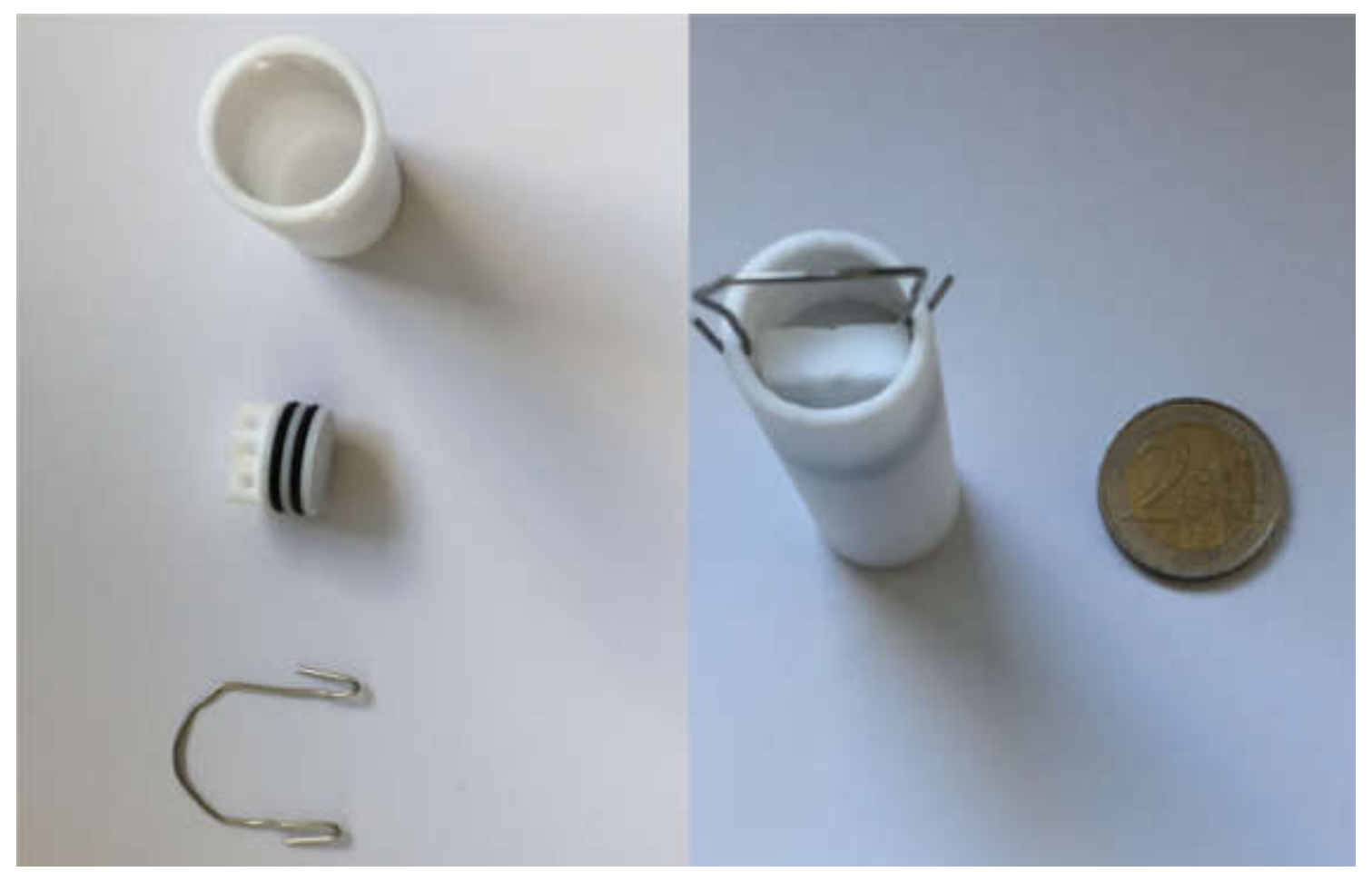

Figure 1: Teflon cylinder for high pressure reactions.

The triisopropylsilyl-derivative of $\mathbf{2 7}$ was synthesized analogusly to the TBS derivatives. For detailed information and spectroscopic data refer to the PhD thesis of E. Geist. ${ }^{1}$ 


\section{Chemical Synthesis}

\section{(S)-2-(Benzyloxy)-1-(pyrrolidin-1-yl)propan-1-one (SI-1)}

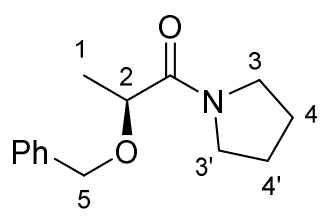

SI-1

Ethyl (S)-lactate (19.3 mL, $0.17 \mathrm{~mol}, 1.0$ eq) was added to pyrrolidine $(15.5 \mathrm{~mL}, 0.19 \mathrm{~mol}, 1.1 \mathrm{eq})$ at $0{ }^{\circ} \mathrm{C}$. The mixture was stirred for $15 \mathrm{~min}$ at $0{ }^{\circ} \mathrm{C}$ and for 3 days at $\mathrm{rt}$. After the removal of the volatiles under reduced pressure the crude amide $(24.20 \mathrm{~g}, 0.17 \mathrm{~mol}, 100 \%)$ was added to a solution of benzylchloride $(32.00 \mathrm{~mL}, 0.28 \mathrm{~mol}, 1.6 \mathrm{eq})$ and Aliquat $336(4.00 \mathrm{~mL}, 0.008 \mathrm{~mol}, 0.05 \mathrm{eq})$ in toluene $(45 \mathrm{~mL})$. The resulting solution was slowly added to a suspension of $\mathrm{NaOH}(23.90 \mathrm{~g}, 0.60 \mathrm{~mol}, 3.5 \mathrm{eq})$ in toluene $(60 \mathrm{~mL})$ at $0{ }^{\circ} \mathrm{C}$. The mixture was stirred for $14 \mathrm{~h}$ at $\mathrm{rt}$ and diluted with $400 \mathrm{~mL} \mathrm{Et}{ }_{2} \mathrm{O}$ and $200 \mathrm{~mL}$ water. Layers were separated and the organic layer was washed with $1 \mathrm{M} \mathrm{HCl}$, a sat. aq. $\mathrm{NaHCO}_{3}$ solution and a sat. aq. $\mathrm{NaCl}$ solution and dried $\left(\mathrm{MgSO}_{4}\right)$. After filtration and removal of the solvent under reduced pressure the crude product was purified by column chromatography (PEEtOAc $=2: 3)$ to afford benzylated amide $\mathbf{S I - 1}(28.17 \mathrm{~g}, 0.12 \mathrm{~mol}, 71 \%)$ as a yellow solid.

$\mathbf{R}_{\mathbf{f}}=0.21$ (PE-EtOAc 1:1); $[\alpha]^{28}{ }_{\mathrm{D}}=-60.5^{\circ}\left(c=1.00 ; \mathrm{CHCl}_{3}\right) ;$ m.p. $=39.8-40.1{ }^{\circ} \mathrm{C} ;{ }^{1} \mathrm{H}-\mathrm{NMR}(200 \mathrm{MHz} ;$ $\left.\mathrm{CDCl}_{3} ; \mathrm{CHCl}_{3}=7.26 \mathrm{ppm}\right) \delta_{\mathrm{H}}=7.24-7.43(5 \mathrm{H}, \mathrm{m}, \mathrm{Ph}), 4.62\left(1 \mathrm{H}, \mathrm{d}, J=11.7 \mathrm{~Hz}, \mathrm{H}_{5}\right), 4.43(1 \mathrm{H}, \mathrm{d}, J=11.7$ $\left.\mathrm{Hz}, \mathrm{H}_{5}\right), 4.21\left(1 \mathrm{H}, \mathrm{q}, J=6.7 \mathrm{~Hz}, \mathrm{H}_{2}\right), 3.41-3.55\left(4 \mathrm{H}, \mathrm{m}, \mathrm{H}_{3}\right), 1.76-1.97\left(4 \mathrm{H}, \mathrm{m}, \mathrm{H}_{4}\right), 1.42(3 \mathrm{H}, \mathrm{d}, J=6.7 \mathrm{~Hz}$, $\mathrm{H}_{1}$ ); ${ }^{13} \mathrm{C}-\mathrm{NMR}\left(101 \mathrm{MHz} ; \mathrm{CDCl}_{3} ; \mathrm{CDCl}_{3}=77.16 \mathrm{ppm}\right) \delta_{\mathrm{C}}=170.9$ (s, $\left.\mathrm{C}_{1}\right), 137.9$ (s, Ph), 128.5 (d, Ph), 128.1 (d, Ph), 127.9 (d, Ph), 74.9 (d, $\left.C_{2}\right), 71.1\left(t, C_{5}\right), 46.4\left(t, C_{3}\right), 46.1\left(t, C_{3^{\prime}}\right), 26.5\left(t, C_{4}\right), 23.8\left(t, C_{4^{\prime}}\right)$, 17.5 (q, $\mathrm{C}_{1}$ ); HRMS (ESI): $\mathrm{m} / z$ calc. for $\mathrm{C}_{14} \mathrm{H}_{19} \mathrm{NO}_{2}[\mathrm{M}+\mathrm{Na}]^{+}: 256.1313$, found 256.1309.

The analytical data match those reported in the literature. ${ }^{2}$ 


\section{(S)-2-(Benzyloxy)pentan-3-one (17)}<smiles>CCC(=O)C(I)OCc1ccccc1</smiles>

Amide SI-1 (30,00 g, $129 \mathrm{mmol}, 1.0 \mathrm{eq})$ was dissolved in dry THF $(300 \mathrm{~mL})$ and cooled to $-30{ }^{\circ} \mathrm{C}$. Ethylmagnesium bromide $3 \mathrm{M}$ solution in $\mathrm{Et}_{2} \mathrm{O}(42.9 \mathrm{~mL}, 129 \mathrm{mmol}, 1.0 \mathrm{eq})$ was slowly added. The mixture was allowed to warm to $\mathrm{rt}$ and stirring was continued for $1 \mathrm{~h}$. The reaction was terminated by addition of a sat. aq. $\mathrm{NH}_{4} \mathrm{Cl}$ solution. Layers were separated and the organic layer was dried over $\mathrm{MgSO}_{4}$. After filtration and removal of the solvent under reduced pressure the crude product was purified by column chromatography $\left(\mathrm{CH}_{2} \mathrm{Cl}_{2}\right)$ to afford ketone $17(23.7 \mathrm{~g}, 124 \mathrm{mmol}, 96 \%)$ as a colorless oil.

$\mathbf{R}_{\mathbf{f}}=0.64\left(\mathrm{CH}_{2} \mathrm{Cl}_{2}\right) ;[\alpha]^{21}{ }_{\mathrm{D}}=-41.3^{\circ}\left(c=1.00 ; \mathrm{CHCl}_{3}\right) ;{ }^{1} \mathrm{H}-\mathrm{NMR}\left(200 \mathrm{MHz} ; \mathrm{CDCl}_{3} ; \mathrm{CHCl}_{3}=7.26 \mathrm{ppm}\right) \delta_{\mathrm{H}}=$ 7.29-7.41 (5H, m, Ph), 4.48-4.60 (2H, m, $\left.\mathrm{H}_{5}\right), 3.96\left(1 \mathrm{H}, \mathrm{q}, J=6.8 \mathrm{~Hz}, \mathrm{H}_{2}\right), 2.55-2.67\left(2 \mathrm{H}, \mathrm{m}, \mathrm{H}_{3}\right), 1.36$ $\left(3 \mathrm{H}, \mathrm{d}, J=6.8 \mathrm{~Hz}, \mathrm{H}_{1}\right), 1.07\left(3 \mathrm{H}, \mathrm{t}, J=7.3 \mathrm{~Hz}, \mathrm{H}_{4}\right) ;{ }^{13} \mathrm{C}-\mathrm{NMR}\left(101 \mathrm{MHz} ; \mathrm{CDCl}_{3} ; \mathrm{CDCl}_{3}=77.16 \mathrm{ppm}\right) \delta_{\mathrm{C}}=$ 213.7 (s, C=O), 137.8 (s, Ph), 128.6 (d, Ph), 128.0 (d, Ph), 127.9 (d, Ph), 80.7 (d, $\left.C_{2}\right), 72.0\left(t, C_{5}\right), 30.7$ (t, $\left.\mathrm{C}_{4}\right), 17.7$ (q, $\mathrm{C}_{1}$ ), 7.4 (q, $\mathrm{C}_{4}$ ); HRMS (ESI): $\mathrm{m} / z$ calc. for $\mathrm{C}_{12} \mathrm{H}_{16} \mathrm{O}_{2}[\mathrm{M}+\mathrm{Na}]^{+}: 215.1048$, found 215.1048 .

The analytical data match those reported in the literature. ${ }^{2}$ 


\section{3-(Triisopropylsilyl)propiolaldehyde (18)}

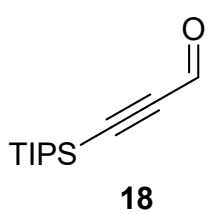

A $2.5 \mathrm{M}$ butyllithium solution in hexane $(9.92 \mathrm{~mL}, 24.8 \mathrm{mmol}, 1.1 \mathrm{eq})$ was added dropwise to a solution of triisopropylsilylacetylene $(5.0 \mathrm{~mL}, 22.5 \mathrm{mmol}, 1.0 \mathrm{eq})$ in dry THF $(50 \mathrm{~mL})$ at $0{ }^{\circ} \mathrm{C}$. The mixture was stirred for $20 \mathrm{~min}$ at $0{ }^{\circ} \mathrm{C}$, for $20 \mathrm{~min}$ at $\mathrm{rt}$ and then cooled to $0{ }^{\circ} \mathrm{C}$. DMF (1.92 mL, 24.8 $\mathrm{mmol}, 1.1 \mathrm{eq}$ ) was slowly added and the mixture was stirred for $20 \mathrm{~min}$ at $\mathrm{rt}$ and then under refluxing conditions for $1 \mathrm{~h}$. After cooling to $0{ }^{\circ} \mathrm{C}$, the mixture was transferred to a slightly acidic $\mathrm{pH}$ value by addition of $5 \% \mathrm{H}_{2} \mathrm{SO}_{4}$ and then weakly basic by addition of a sat. aq. $\mathrm{NaHCO}_{3}$ solution. The mixture was extracted with $\mathrm{CH}_{2} \mathrm{Cl}_{2}$ and the combined organic phases were dried over $\mathrm{MgSO}_{4}$, filtered and evaporated under reduced pressure. The aldehyde 18 (4.73 g, $22.5 \mathrm{mmol}, 100 \%)$ was used without further purification.

$\mathbf{R}_{\mathbf{f}}=0.20(\mathrm{PE}) ;{ }^{1} \mathrm{H}-\mathrm{NMR}\left(200 \mathrm{MHz} ; \mathrm{CDCl}_{3} ; \mathrm{CHCl}_{3}=7.26 \mathrm{ppm}\right) \delta_{\mathrm{H}}=9.23(1 \mathrm{H}, \mathrm{s}, \mathrm{CHO}), 1.04-1.20(21 \mathrm{H}, \mathrm{m}$, TIPS).

The analytical data match those reported in the literature. ${ }^{3}$ 
<smiles>C[C@H](OCc1ccccc1)C(=O)[C@@H](C)[C@@H](I)C#C[I-]</smiles>

SI-2

Titanium tetrachloride $(8.00 \mathrm{~mL}, 72.82 \mathrm{mmol}, 1.1 \mathrm{eq})$ and diisopropylethylamine $(12.7 \mathrm{~mL}$, $72.82 \mathrm{mmol}, 1.1 \mathrm{eq}$ ) were successively added dropwise to a solution of ketone $17(12.7 \mathrm{~g}, 66.2 \mathrm{mmol}$, 1.0 eq) in dry $\mathrm{CH}_{2} \mathrm{Cl}_{2}(400 \mathrm{~mL})$ at $-78^{\circ} \mathrm{C}$. The mixture was stirred for $1.5 \mathrm{~h}$ at $-78^{\circ} \mathrm{C}$, then a solution of aldehyde 18 (19.5 g, $92.68 \mathrm{mmol}, 1.4 \mathrm{eq})$ in $\mathrm{CH}_{2} \mathrm{Cl}_{2}(20 \mathrm{ml})$ was added over a period of $45 \mathrm{~min}$ and stirring was continued for $1 \mathrm{~h}$. The reaction was terminated by addition of a sat. aq. $\mathrm{NH}_{4} \mathrm{Cl}$ solution which was followed by dilution with $\mathrm{Et}_{2} \mathrm{O}$ and water. Layers were separated and the aqueous layer was extracted with $\mathrm{Et}_{2} \mathrm{O}$. The combined organic phases were washed with a solution of a sat. aq. $\mathrm{NaHCO}_{3}$, with brine followed by drying over $\mathrm{MgSO}_{4}$. After filtration and removal of the solvent under reduced pressure the crude product was purified by column chromatography (PE-EtOAc= 25:1 $\rightarrow$ 10:1) to afford aldol SI-2 (26.40 g, $63.55 \mathrm{mmol}, 96 \%, 12: 1 \mathrm{dr})$ as a colorless oil.

$\mathbf{R}_{\mathbf{f}}=0.15\left(\right.$ PE-EtOAC 10:1); $[\alpha]^{22} \mathrm{D}=-10.7^{\circ}\left(c=1.00 ; \mathrm{CHCl}_{3}\right) ;{ }^{1} \mathrm{H}-\mathrm{NMR}\left(400 \mathrm{MHz} ; \mathrm{CDCl}_{3} ; \mathrm{CHCl}_{3}=7.26\right.$ ppm) $\delta_{\mathrm{H}}=7.31-7.41(5 \mathrm{H}, \mathrm{m}, \mathrm{Ph}), 4.68\left(1 \mathrm{H}, \mathrm{d}, J=4.3 \mathrm{~Hz}, \mathrm{H}_{4}\right), 4.51-4.64\left(2 \mathrm{H}, \mathrm{m}, \mathrm{H}_{5}\right), 4.11(1 \mathrm{H}, \mathrm{q}, J=6.8$ $\left.\mathrm{Hz}, \mathrm{H}_{2}\right), 3.14-3.29\left(1 \mathrm{H}, \mathrm{m}, \mathrm{H}_{3}\right), 1.40\left(3 \mathrm{H}, \mathrm{d}, J=6.8 \mathrm{~Hz}, \mathrm{H}_{1}\right), 1.32\left(3 \mathrm{H}, \mathrm{d}, J=7.2 \mathrm{~Hz}, \mathrm{H}_{6}\right), 1.04(21 \mathrm{H}, \mathrm{s}$, TIPS); ${ }^{13} \mathrm{C}-\mathrm{NMR}\left(101 \mathrm{MHz} ; \mathrm{CDCl}_{3} ; \mathrm{CDCl}_{3}=77.16 \mathrm{ppm}\right) \delta_{\mathrm{C}}=213.8(\mathrm{~s}, \mathrm{C}=0), 137.5(\mathrm{~s}, \mathrm{Ph}), 128.6(\mathrm{~d}, \mathrm{Ph})$, 128.0 (d, Ph), 127.8 (d, Ph), $106.3\left(\mathrm{~s}, \mathrm{C}_{7}\right), 86.4$ (s, $\mathrm{C}_{8}$ ), 79.5 (d, $\mathrm{C}_{2}$ ), 72.0 (t, $\left.\mathrm{C}_{5}\right), 63.8\left(\mathrm{~d}, \mathrm{C}_{4}\right), 47.8\left(\mathrm{~d}, \mathrm{C}_{3}\right)$, 18.6 (d, TIPS), 17.2 (q, $\mathrm{C}_{1}$ ), 11.9 (q, $\mathrm{C}_{6}$ ), 11.3 (q. TIPS) (TIPS, $\mathrm{C}_{1}, \mathrm{C}_{6}$ ). HRMS (ESI): $\mathrm{m} / z$ calc. for $\mathrm{C}_{24} \mathrm{H}_{38} \mathrm{O}_{3} \mathrm{Si}$ $[\mathrm{M}+\mathrm{Na}]^{+}:$425.2488, found 425.2488 . 


\section{(3S,4R,5R,6S)-6-(Benzyloxy)-4-methyl-1-(triisopropylsilyl)hept-1-yne-3,5-diol (SI-3)}<smiles>CC(C#CC(F)F)C(O)C(C)C(C)OCc1ccccc1</smiles>

Tetrasodium triacetoxyborohydride $(2.03 \mathrm{~g}, 9.6 \mathrm{mmol}, 2.00 \mathrm{eq})$ and dry AcOH $(0.88 \mathrm{ml}, 15.36 \mathrm{mmol}$, $3.2 \mathrm{eq})$. were added to a solution of aldol SI-2 $(2.00 \mathrm{~g}, 4.8 \mathrm{mmol}, 1.0 \mathrm{eq})$. in MeCN (44 ml) and the mixture was stirred for $4.5 \mathrm{~h}$ at $\mathrm{rt}$. The reaction was terminated by addition of a sat. aq. potassiumsodium tartrate solution and stirring was continued for $20 \mathrm{~min}$ at rt. Layers were separated and the aqueous layer was extracted with EtOAc. The combined organic phases were dried over $\mathrm{MgSO}_{4}$, filtered and evaporated under reduced pressure. Column chromatography (PE-EtOAc= 20:1 $\rightarrow$ 15:1 $\rightarrow$ 10:1) of the crude product afforded diol SI-3 (1.86 g, $4.5 \mathrm{mmol}, 93 \%, 10: 1 \mathrm{dr}$ ) as a colorless oil.

$\mathbf{R}_{\mathbf{f}}=0.49$ (PE-EtOAc 5:1); $[\alpha]^{23}{ }_{\mathrm{D}}=-8.8^{\circ}\left(c=1.00 ; \mathrm{CHCl}_{3}\right) ;{ }^{1} \mathrm{H}-\mathrm{NMR}\left(400 \mathrm{MHz} ; \mathrm{CDCl}_{3} ; \mathrm{CHCl}_{3}=7.26 \mathrm{ppm}\right)$ $\delta_{H}=7.31-7.41(5 \mathrm{H}, \mathrm{m}, \mathrm{Ph}), 4.59\left(2 \mathrm{H}, \mathrm{s}, \mathrm{H}_{7}\right), 4.47\left(1 \mathrm{H}, \mathrm{d}, J=3.1 \mathrm{~Hz}, \mathrm{H}_{5}\right), 4.24(1 \mathrm{H}, \mathrm{dd}, J=9.4,2.9 \mathrm{~Hz}$, $\left.\mathrm{H}_{3}\right), 3.62\left(1 \mathrm{H}, \mathrm{dq}, J=6.3,2.9 \mathrm{~Hz}, \mathrm{H}_{2}\right), 1.95-2.03\left(1 \mathrm{H}, \mathrm{m}, \mathrm{H}_{4}\right), 1.20\left(3 \mathrm{H}, \mathrm{d}, J=6.5 \mathrm{~Hz}, \mathrm{H}_{1}\right), 1.11(21 \mathrm{H}, \mathrm{s}$, TIPS), $0.91\left(3 \mathrm{H}, \mathrm{d}, J=7.2 \mathrm{~Hz}, \mathrm{H}_{6}\right) ;{ }^{13} \mathrm{C}-\mathrm{NMR}\left(101 \mathrm{MHz} ; \mathrm{CDCl}_{3} ; \mathrm{CDCl}_{3}=77.16 \mathrm{ppm}\right) \delta_{\mathrm{c}}=138.3(\mathrm{~s}, \mathrm{Ph})$, 128.6 (d, Ph), 127.9 (d, Ph), 127.7 (d, Ph), 107.2 (s, C8), 86.5 (s, C9), 75.4 (d, $C_{2}$ ), 74.9 (t, $C_{7}$ ), 70.6 (d, $\left.C_{3}\right), 67.9$ (d, $C_{5}$ ), 39.6 (d, $C_{4}$ ), 18.8 (d, TIPS), 12.8 (q, $C_{6}$ ), 12.6 (q, $C_{1}$ ), 11.3 (q, TIPS). HRMS (ESI): m/z calc. for $\mathrm{C}_{24} \mathrm{H}_{40} \mathrm{O}_{3} \mathrm{Si}[\mathrm{M}+\mathrm{Na}]^{+}$: 427.2644 , found 427.2647 . 
<smiles>C#CC(O)C(C)C(O)C(C)OCc1ccccc1</smiles>

19

A solution (1M) of tetrabutylammonium fluoride in THF ( $8.5 \mathrm{~mL}, 8.5 \mathrm{mmol}, 1.1 \mathrm{eq})$ was added to a solution of diol SI-3 (3.12 g, $7.7 \mathrm{mmol}, 1.0 \mathrm{eq})$ in THF $(30 \mathrm{~mL})$ at $0{ }^{\circ} \mathrm{C}$. The mixture was stirred for $3 \mathrm{~h}$ at $50{ }^{\circ} \mathrm{C}$ and terminated by addition of a sat. aq. $\mathrm{NH}_{4} \mathrm{Cl}$ solution. Layers were separated and the aqueous layer was extracted with EtOAc. The combined organic phases were dried over $\mathrm{MgSO}_{4}$, filtered and evaporated under reduced pressure. Column chromatography (PE-EtOAc= 5:1 $\rightarrow$ 2:1) of the crude product afforded diol 19 (1.93 g, $7.7 \mathrm{mmol}$, quant.) as a colorless oil.

$\mathbf{R}_{\mathbf{f}}=0.14\left(\right.$ PE-EtOAc 2:1); $[\alpha]^{22}{ }_{\mathrm{D}}=-7.6^{\circ}\left(c=1.00 ; \mathrm{CHCl}_{3}\right) ;{ }^{1} \mathrm{H}-\mathrm{NMR}\left(400 \mathrm{MHz} ; \mathrm{CDCl}_{3} ; \mathrm{CHCl}_{3}=7.26 \mathrm{ppm}\right)$ $\delta_{\mathrm{H}}=7.31-7.41(5 \mathrm{H}, \mathrm{m}, \mathrm{Ph}), 4.57-4.68\left(2 \mathrm{H}, \mathrm{m}, \mathrm{H}_{9}\right), 4.46\left(1 \mathrm{H}, \mathrm{s}, \mathrm{H}_{5}\right), 4.16\left(1 \mathrm{H}, \mathrm{dd}, J=9.6,3.1 \mathrm{~Hz}, \mathrm{H}_{3}\right)$, $3.63\left(1 \mathrm{H}, \mathrm{dq}, J=6.3,2.9 \mathrm{~Hz}, \mathrm{H}_{2}\right), 2.50\left(1 \mathrm{H}, \mathrm{d}, J=2.1 \mathrm{~Hz}, \mathrm{H}_{7}\right), 1.95-2.03\left(1 \mathrm{H}, \mathrm{m}, \mathrm{H}_{4}\right), 1.20(3 \mathrm{H}, \mathrm{d}, J=6.1$ $\left.\mathrm{Hz}, \mathrm{H}_{1}\right), 0.90\left(3 \mathrm{H}, \mathrm{d}, J=7.2 \mathrm{~Hz}, \mathrm{H}_{8}\right) ;{ }^{13} \mathrm{C}-\mathrm{NMR}\left(101 \mathrm{MHz} ; \mathrm{CDCl}_{3} ; \mathrm{CDCl}_{3}=77.16 \mathrm{ppm}\right) \delta_{\mathrm{C}}=138.2(\mathrm{~s}, \mathrm{Ph})$, 128.7 (d, Ph), 128.0 (d, Ph), 127.8 (d, o-Ph), 83.3 (s, C6), 75.4 (d, $C_{2}$ ), 75.0 (d, $C_{7}$ ), 74.0 (d, $C_{3}$ ), 70.8 (t, $\left.\mathrm{C}_{9}\right), 67.2\left(\mathrm{~d}, \mathrm{C}_{5}\right), 39.3\left(\mathrm{~d}, \mathrm{C}_{4}\right), 12.6\left(\mathrm{q}, \mathrm{C}_{8}\right), 12.4\left(\mathrm{q}, \mathrm{C}_{1}\right)$. HRMS (ESI): $\mathrm{m} / z$ calc. for $\mathrm{C}_{15} \mathrm{H}_{20} \mathrm{O}_{3}[\mathrm{M}+\mathrm{Na}]^{+}$: 271.1310, found 271.1310. 
<smiles>C#CC(O[Sb])C(C)C(O)C(C)OCc1ccccc1</smiles>

SI-4

tert-Butyldimethylsilyl trifluoromethane sulfonate $(1.60 \mathrm{~mL}, 7.0 \mathrm{mmol}, 0.95 \mathrm{eq})$ was slowly added to a solution of diol 19 (1.83 g, $7.4 \mathrm{mmol}, 1.0 \mathrm{eq}$ ) and 2,6-lutidine (1.28 mL, $11.1 \mathrm{mmol}, 1.5 \mathrm{eq})$ in $\mathrm{CH}_{2} \mathrm{Cl}_{2}$ at $0{ }^{\circ} \mathrm{C}$. The mixture was stirred for $2 \mathrm{~h}$ at $0{ }^{\circ} \mathrm{C}$ and terminated by addition of a sat. aq. $\mathrm{NaHCO}_{3}$ solution. Layers were separated and the aqueous layer was extracted with $\mathrm{CH}_{2} \mathrm{Cl}_{2}$. The combined organic phases were dried over $\mathrm{MgSO}_{4}$. filtered and evaporated under reduced pressure. Column chromatography (PE-EtOAc= 5:1) of the crude product afforded alcohol SI-4 $(2.42 \mathrm{~g}, 6.6 \mathrm{mmol}, 90 \%)$ as a colorless oil.

$\mathbf{R}_{\mathbf{f}}=0.70\left(\right.$ PE-EtOAC 5:1); $[\alpha]^{22}{ }_{\mathrm{D}}=+38.0^{\circ}\left(c=1.00 ; \mathrm{CHCl}_{3}\right) ;{ }^{1} \mathrm{H}-\mathbf{N M R}\left(400 \mathrm{MHz} ; \mathrm{CDCl}_{3} ; \mathrm{CHCl}_{3}=7.26 \mathrm{ppm}\right)$ $\delta_{H}=7.28-7.39(5 \mathrm{H}, \mathrm{m}, \mathrm{Ph}), 4.82-4.84\left(1 \mathrm{H}, \mathrm{m}, \mathrm{H}_{5}\right), 4.49-4.62\left(2 \mathrm{H}, \mathrm{m}, \mathrm{H}_{9}\right), 3.78-3.82\left(1 \mathrm{H}, \mathrm{m}, \mathrm{H}_{3}\right), 3.57$ $\left(1 \mathrm{H}, \mathrm{dq}, J=6.3,3.7 \mathrm{~Hz}, \mathrm{H}_{2}\right), 2.38\left(1 \mathrm{H}, \mathrm{d}, J=2.0 \mathrm{~Hz}, \mathrm{H}_{7}\right), 1.71-1.79\left(1 \mathrm{H}, \mathrm{m}, \mathrm{H}_{4}\right), 1.19(3 \mathrm{H}, \mathrm{d}, J=6.5 \mathrm{~Hz}$, $\left.\mathrm{H}_{1}\right), 0.97\left(3 \mathrm{H}, \mathrm{d}, J=7.2 \mathrm{~Hz}, \mathrm{H}_{8}\right), 0.91(9 \mathrm{H}, \mathrm{s}, \mathrm{TBS}), 0.17(3 \mathrm{H}, \mathrm{s}, \mathrm{TBS}), 0.12(3 \mathrm{H}, \mathrm{s}, \mathrm{TBS}) ;{ }^{13} \mathrm{C}-\mathrm{NMR}(101$ $\left.\mathrm{MHz} ; \mathrm{CDCl}_{3} ; \mathrm{CDCl}_{3}=77.16 \mathrm{ppm}\right) \delta_{\mathrm{c}}=138.6(\mathrm{~s}, \mathrm{Ph}), 128.6(\mathrm{~d}, \mathrm{Ph}), 127.8(\mathrm{~d}, \mathrm{Ph}), 84.9\left(\mathrm{~s}, \mathrm{C}_{6}\right), 75.6(\mathrm{~d}$, $\left.C_{2}\right), 73.0\left(d, C_{3}\right), 72.7\left(d, C_{7}\right), 70.5\left(t, C_{9}\right), 63.6\left(d, C_{5}\right), 41.5\left(d, C_{4}\right), 25.9$ (q, TBS), 18.3 (s,TBS), 12.9 (q, $\mathrm{C}_{1}$ ), 10.2 (q, $\mathrm{C}_{8}$ ), -4.4 (q, TBS), -5.2 (q, TBS). HRMS (ESI): $\mathrm{m} / \mathrm{z}$ calc. for $\mathrm{C}_{21} \mathrm{H}_{34} \mathrm{O}_{3} \mathrm{Si}[\mathrm{M}+\mathrm{Na}]^{+}: 385.2175$, found 385.2173 . 

(20)<smiles>CC1C(O[C@@H](C)C(C)OCc2ccccc2)C=COC1OC(C)(C)C</smiles>

20

To a solution of alkynol SI-4 (1.28 g, $3.53 \mathrm{mmol}, 1.0 \mathrm{eq})$ in dry THF (30 mL) were added tungsten hexacarbonyl ( $0.31 \mathrm{~g}, 0.88 \mathrm{mmol}, 0.25 \mathrm{eq})$ and triethylamine $(7.00 \mathrm{~mL}, 52 \mathrm{mmol}, 15 \mathrm{eq})$. The mixture was degassed for $30 \mathrm{~min}$ and afterwards heated under refluxing conditions and irradiated with a lowpressure mercury lamp under an inert atmosphere over a period of $6 \mathrm{~h}$. The mixture was filtered over Celite ${ }^{\circledR}$ and the volatile components were removed under reduced pressure. Column chromatography (PE-EtOAc= 50:1 $\rightarrow$ 20:1) of the crude product afforded dihydropyran 20 (1.05 g, 2.9 $\mathrm{mmol}, 82 \%)$ as a colorless oil.

$\mathbf{R}_{\mathbf{f}}=0.26\left(\right.$ PE-EtOAC 20:1); $[\alpha]^{23}{ }_{\mathrm{D}}=+80.2^{\circ}\left(c=1.00 ; \mathrm{CHCl}_{3}\right) ;{ }^{1} \mathrm{H}-\mathrm{NMR}\left(400 \mathrm{MHz} ; \mathrm{CDCl}_{3} ; \mathrm{CHCl}_{3}=7.26\right.$ ppm) $\delta_{H}=7.42-7.27(5 \mathrm{H}, \mathrm{m}, \mathrm{Ph}), 6.39\left(1 \mathrm{H}, \mathrm{dd}, J=6.2,1.3 \mathrm{~Hz}, \mathrm{H}_{1}\right), 4.65\left(1 \mathrm{H}, \mathrm{dd}, J=6.2,2.3 \mathrm{~Hz}, \mathrm{H}_{2}\right)$, $4.61\left(1 \mathrm{H}, \mathrm{d}, J=11.9 \mathrm{~Hz}, \mathrm{H}_{9}\right), 4.56\left(1 \mathrm{H}, \mathrm{d}, J=11.9 \mathrm{~Hz}, \mathrm{H}_{9^{\prime}}\right), 3.94\left(1 \mathrm{H}, \mathrm{ddd}, J=7.5,2.1,1.3 \mathrm{~Hz}, \mathrm{H}_{3}\right), 3.87-$ $3.79\left(2 \mathrm{H}, \mathrm{m}, \mathrm{H}_{5}, \mathrm{H}_{7}\right), 1.86-1.77\left(1 \mathrm{H}, \mathrm{m}, \mathrm{H}_{4}\right), 1.21\left(3 \mathrm{H}, \mathrm{d}, J=6.5 \mathrm{~Hz}, \mathrm{H}_{8}\right), 0.91\left(3 \mathrm{H}, \mathrm{d}, J=5.8 \mathrm{~Hz}, \mathrm{H}_{6}\right)$, 0.91 (9H, s, TBS), 0.09 (3H, s, TBS), 0.08 (3H, s, TBS); ${ }^{13} \mathrm{C}-\mathrm{NMR}\left(101 \mathrm{MHz} ; \mathrm{CDCl}_{3} ; \mathrm{CDCl}_{3}=77.16 \mathrm{ppm}\right) \delta_{\mathrm{c}}$ $=144.1\left(\mathrm{~d}, \mathrm{C}_{1}\right), 138.7(\mathrm{~s}, \mathrm{Ph}), 128.5(\mathrm{~d}, \mathrm{Ph}), 127.8(\mathrm{~d}, \mathrm{Ph}), 127.6(\mathrm{~d}, \mathrm{Ph}), 105.1\left(\mathrm{~d}, \mathrm{C}_{2}\right), 80.3\left(\mathrm{~d}, \mathrm{C}_{7}\right), 73.6$ $\left(d, C_{5}\right), 70.7\left(t, C_{9}\right), 69.5\left(d, C_{3}\right), 37.2\left(d, C_{4}\right), 26.0$ (q, TBS), 18.3 (s, TBS), 14.5 (q, $\left.C_{6}\right), 13.5$ (q, $\left.C_{8}\right),-4.0$ (q, TBS), -4.4 (q, TBS); HRMS (ESI): $m / z$ calc. for $\mathrm{C}_{21} \mathrm{H}_{34} \mathrm{O}_{3} \mathrm{Si}[\mathrm{M}+\mathrm{Na}]^{+}: 385.2175$, found 385.2174. 
(4R,5R,6R)-4-((S)-1-(Benzyloxy)ethyl)-6-ethynyl-2,2,5-trimethyl-1,3-dioxane (SI-5)

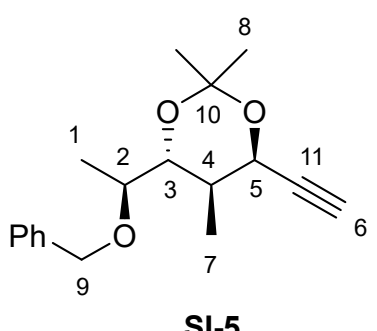

Diol 19 (690 mg, $2.78 \mathrm{mmol}, 1.0 \mathrm{eq})$ and camphorsulfonic acid (6 mg, $0.28 \mathrm{mmol}, 0.1 \mathrm{eq}$ ) were dissolved in 3,3-dimethoxypropane (15 mL) and the resulting mixture was stirred for $14 \mathrm{~h}$ at $\mathrm{rt}$. After removal of the solvent under reduced pressure the crude product was purified by column chromatography (PE-EtOAc 20:1 $\rightarrow$ 10:1) to afford acetonide SI-5 (108 mg, $0.37 \mathrm{mmol}, 98 \%$ ) as a colorless oil.

$\mathbf{R}_{\mathbf{f}}=0.17\left(\right.$ PE-EtOAc 20:1); $[\alpha]^{23}{ }_{\mathrm{D}}=-7.5^{\circ}\left(c=1.00 ; \mathrm{CHCl}_{3}\right) ;{ }^{1} \mathrm{H}-\mathrm{NMR}\left(400 \mathrm{MHz} ; \mathrm{CDCl}_{3} ; \mathrm{CHCl}_{3}=7.26 \mathrm{ppm}\right)$ $\delta_{H}=7.29-7.38(5 \mathrm{H}, \mathrm{m}, \mathrm{Ph}), 4.55-4.67\left(3 \mathrm{H}, \mathrm{m}, \mathrm{H}_{5}, \mathrm{H}_{9}\right), 3.54-3.62\left(2 \mathrm{H}, \mathrm{m}, \mathrm{H}_{2}, \mathrm{H}_{3}\right), 1.95-2.03\left(1 \mathrm{H}, \mathrm{m}, \mathrm{H}_{4}\right)$, $1.49\left(3 \mathrm{H}, \mathrm{s}, \mathrm{H}_{8}\right), 1.40\left(3 \mathrm{H}, \mathrm{s}, \mathrm{H}_{8}\right), 1.23\left(3 \mathrm{H}, \mathrm{d}, J=5.9 \mathrm{~Hz}, \mathrm{H}_{1}\right), 1.09\left(3 \mathrm{H}, \mathrm{d}, J=7.1 \mathrm{~Hz}, \mathrm{H}_{7}\right) ;{ }^{13} \mathrm{C}-\mathrm{NMR}(101$ $\mathrm{MHz} ; \mathrm{CDCl}_{3} ; \mathrm{CDCl}_{3}=77.16$ ppm) $\delta_{\mathrm{c}}=138.2(\mathrm{~s}, \mathrm{Ph}), 128.5$ (d, Ph), $127.8(\mathrm{~d}, \mathrm{Ph}), 127.7$ (d, Ph), 101.0 (s, $\left.\mathrm{C}_{10}\right), 81.2\left(\mathrm{~s}, \mathrm{C}_{11}\right), 76.2\left(\mathrm{~d}, \mathrm{C}_{3}\right), 75.8\left(\mathrm{~d}, \mathrm{C}_{2}\right), 75.6\left(\mathrm{~d}, \mathrm{C}_{6}\right), 71.2\left(\mathrm{t}, \mathrm{C}_{9}\right), 63.2\left(\mathrm{~d}, \mathrm{C}_{5}\right), 36.0\left(\mathrm{~d}, \mathrm{C}_{4}\right), 27.0\left(\mathrm{q}, \mathrm{C}_{8}\right)$, $23.4\left(q, C_{8}\right), 15.5$ (q, $\left.C_{1}\right), 14.1$ (q, $\left.C_{7}\right)$; HRMS (ESI): $m / z$ calc. for $\mathrm{C}_{18} \mathrm{H}_{24} \mathrm{O}_{3}[\mathrm{M}+\mathrm{Na}]^{+}:$311.1623, found 311.1627. 


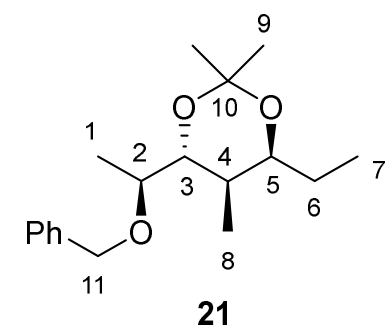

To a solution of acetonide SI-5 (108 mg, $0.37 \mathrm{mmol}, 1.0$ Äq) in MeOH (3 mL) was added a catalytic amount of $5 \% \mathrm{Pd} / \mathrm{C}(4.3 \mathrm{mg})$ and ammonium acetate $(10 \mathrm{mg}, 0.13 \mathrm{mmol}, 0.28 \mathrm{Äq})$. The mixture was stirred at rt under an hydrogen atmosphere $(1 \mathrm{~atm})$ for $16 \mathrm{~h}$. Filtration through Celite ${ }^{\circledR}$ and removal of the solvent gave a residue which was purified by column chromatography (PE-EtOAc 20:1 $\rightarrow$ 10:1) to afford acetonide 21 (61 mg, $0.21 \mathrm{mmol}, 56 \%$ ) as a colorless oil.

${ }^{1} \mathrm{H}-\mathrm{NMR}\left(400 \mathrm{MHz} ; \mathrm{C}_{6} \mathrm{D}_{6} ; \mathrm{C}_{6} \mathrm{D}_{5} \mathrm{H}=7.16 \mathrm{ppm}\right) \delta_{\mathrm{H}}=7.34(2 \mathrm{H}, \mathrm{d}, J=7.5 \mathrm{~Hz}, \mathrm{Ph}), 7.08-7.21(3 \mathrm{H}, \mathrm{m}, \mathrm{Ph})$, $4.53\left(1 \mathrm{H}, \mathrm{d}, J=11.9 \mathrm{~Hz}, \mathrm{H}_{11}\right), 4.37\left(1 \mathrm{H}, \mathrm{d}, J=11.9 \mathrm{~Hz}, \mathrm{H}_{11}\right), 3.67-3.71\left(1 \mathrm{H}, \mathrm{m}, \mathrm{H}_{5}\right), 3.48(1 \mathrm{H}, \mathrm{q}, J=5.8$ $\left.\mathrm{Hz}, \mathrm{H}_{2}\right), 3.41\left(1 \mathrm{H}, \mathrm{dd}, J=6.8,5.1 \mathrm{~Hz}, \mathrm{H}_{3}\right), 1.76-1.85\left(1 \mathrm{H}, \mathrm{m}, \mathrm{H}_{4}\right), 1.45-1.56\left(1 \mathrm{H}, \mathrm{m}, \mathrm{H}_{6}\right), 1.37\left(6 \mathrm{H}, \mathrm{s}, \mathrm{H}_{9}\right)$, $1.25\left(3 \mathrm{H}, \mathrm{d}, J=6.1 \mathrm{~Hz}, \mathrm{H}_{1}\right), 1.15-1.28\left(1 \mathrm{H}, \mathrm{m}, \mathrm{H}_{6}\right), 0.95\left(3 \mathrm{H}, \mathrm{d}, J=6.5 \mathrm{~Hz}, \mathrm{H}_{8}\right), 0.90(3 \mathrm{H}, \mathrm{t}, J=7.3 \mathrm{~Hz}$, $\mathrm{H}_{7}$ ); ${ }^{13} \mathrm{C}-\mathrm{NMR}\left(101 \mathrm{MHz} ; \mathrm{C}_{6} \mathrm{D}_{6} ; \mathrm{C}_{6} \mathrm{D}_{6}=127.06 \mathrm{ppm}\right) \delta_{\mathrm{C}}=138.9$ (q, Ph), $128.4(\mathrm{t}, \mathrm{Ph}), 127.9(\mathrm{t}, \mathrm{Ph}), 127.7$ (t, Ph), 100.4 (q, $\left.C_{10}\right), 78.1,77.7,71.2,71.1\left(t, C_{2}, C_{3}, C_{5}, C_{11}\right), 36.7\left(t, C_{4}\right), 25.6\left(s, C_{6}\right), 24.0,23.9\left(p, C_{9}\right)$, $16.4,13.2\left(p, C_{1}, C_{8}\right), 10.8\left(p, C_{7}\right)$. 
(2R,3S,4R)-2-((S)-1-(Benzyloxy)ethyl)-4-(tert-butyldimethylsilyloxy)-5-bromo-3-methyl-3,4-dihydro$2 H$-pyran (SI-6)<smiles>CC(C)[C@H]1OC=C(Br)[C@@H](OCc2ccccc2)[C@H]1C</smiles>

A solution of bromine $(0.152 \mathrm{~g}, 0.95 \mathrm{mmol}, 1.0 \mathrm{eq})$ in $\mathrm{CH}_{2} \mathrm{Cl}_{2}(3 \mathrm{ml})$ was slowly added to a solution of enol ether 20 ( $0.344 \mathrm{~g}, 0.95 \mathrm{mmol}, 1.0 \mathrm{eq})$ in $\mathrm{CH}_{2} \mathrm{Cl}_{2}(5 \mathrm{~mL})$ at $-78^{\circ} \mathrm{C}$. Towards the end of addition the reaction mixture turned yellow and no starting material could be detected as judged by TLC. The mixture was allowed to warm to rt and triethylamine $(1.04 \mathrm{~mL}, 7.60 \mathrm{mmol}, 8 \mathrm{eq})$ was added. The mixture was stirred for $48 \mathrm{~h}$ at rt and then diluted with EtOAc and washed with a sat. aq. $\mathrm{Na}_{2} \mathrm{~S}_{2} \mathrm{O}_{3}$ solution. Layers were separated, the organic layer was dried over $\mathrm{MgSO}_{4}$, filtered and evaporated under reduced pressure. Column chromatography $\left(\mathrm{PE}-\mathrm{CH}_{2} \mathrm{Cl}_{2}=10: 1 \rightarrow 5: 1\right)$ of the crude product afforded bromide SI-6 (0.202 g, $0.46 \mathrm{mmol}, 48 \%$ ) as a colorless oil.

$\mathbf{R}_{\mathbf{f}}=0.54\left(\mathrm{PE}-\mathrm{CH}_{2} \mathrm{Cl}_{2} 1: 1\right) ;[\alpha]^{21}{ }_{\mathrm{D}}=+36.6^{\circ}\left(c=1.00 ; \mathrm{CHCl}_{3}\right) ;{ }^{1} \mathrm{H}-\mathrm{NMR}\left(400 \mathrm{MHz} ; \mathrm{CDCl}_{3} ; \mathrm{CHCl}_{3}=7.26\right.$ ppm) $\delta_{H}=7.25-7.37(5 \mathrm{H}, \mathrm{m}, \mathrm{Ph}), 6.62\left(1 \mathrm{H}, \mathrm{s}, \mathrm{H}_{1}\right), 4.52-4.62\left(2 \mathrm{H}, \mathrm{m}, \mathrm{H}_{9}\right), 4.11-4.17\left(1 \mathrm{H}, \mathrm{m}, \mathrm{H}_{7}\right), 3.91$ $\left(1 \mathrm{H}, \mathrm{d}, J=3.4 \mathrm{~Hz}, \mathrm{H}_{3}\right), 3.78\left(1 \mathrm{H}, \mathrm{dd}, J=7.2,4.4 \mathrm{~Hz}, \mathrm{H}_{5}\right), 2.43-2.50\left(1 \mathrm{H}, \mathrm{m}, \mathrm{H}_{4}\right), 1.23(3 \mathrm{H}, \mathrm{d}, J=6.1 \mathrm{~Hz}$, $\left.\mathrm{H}_{8}\right), 1.04\left(3 \mathrm{H}, \mathrm{d}, J=7.2 \mathrm{~Hz}, \mathrm{H}_{6}\right), 0.93(9 \mathrm{H}, \mathrm{s}, \mathrm{TBS}), 0.21$ (3H, s, TBS), $0.12(3 \mathrm{H}, \mathrm{s}, \mathrm{TBS}) ;{ }^{13} \mathrm{C}-\mathrm{NMR}(101$ $\mathrm{MHz} ; \mathrm{CDCl}_{3} ; \mathrm{CDCl}_{3}=77.16$ ppm) $\delta_{\mathrm{C}}=142.9$ (d, $\left.\mathrm{C}_{1}\right) 138.9$ (s, Ph), 128.5 (d, Ph), 127.6 (d, Ph), 127.4 (d, Ph), 102.6 (s, $\left.C_{2}\right), 80.9\left(d, C_{5}\right), 72.6\left(d, C_{7}\right), 72.2\left(d, C_{3}\right), 70.3$ (t, $\left.C_{9}\right), 37.8\left(d, C_{4}\right), 26.3$ (q, TBS), 18.6 (s, TBS), 16.9 (q, $\mathrm{C}_{6}$ ), 15.7 (q, $\mathrm{C}_{8}$ ), -3.5 (q, TBS), -4.0 (q, TBS); HRMS (ESI): $\mathrm{m} / \mathrm{z}$ calc. for $\mathrm{C}_{21} \mathrm{H}_{33} \mathrm{O}_{3} \mathrm{SiBr}$ $[\mathrm{M}+\mathrm{Na}]^{+}:$463.1280, found 463.1290 . 
<smiles>C=CC1=COC(C(C)OCc2ccccc2)[C@H](C)[C@@H]1OC(C)C</smiles>

Tris(dibenzylideneacetone)dipalladium (35.0 mg, $0.039 \mathrm{mmol}$, $0.1 \mathrm{eq}$ ), triphenylarsine (95 mg, 0.31 $\mathrm{mmol}, 0.8 \mathrm{eq})$ and tributyl(vinyl)stannane $(0.22 \mathrm{~mL}, 0.78 \mathrm{mmol}, 2.0 \mathrm{eq})$ were added to a solution of bromide SI-6 (170 mg, $0.39 \mathrm{mmol}, 1.0 \mathrm{eq})$ in dry THF $(4 \mathrm{~mL})$. The mixture was heated under refluxing conditions for $16 \mathrm{~h}$ and filtered through a pad of Celite ${ }^{\circledR}$. After removal of the solvent under reduced pressure the crude product was purified by column chromatography $\left(\mathrm{PE}-\mathrm{CH}_{2} \mathrm{Cl}_{2}=10: 1 \rightarrow 5: 1\right)$ to afford diene 22 (71 $\mathrm{mg}, 0.18 \mathrm{mmol}, 47 \%$ ) as a colorless oil.

$\mathbf{R}_{\mathbf{f}}=0.45\left(\mathrm{PE}-\mathrm{CH}_{2} \mathrm{Cl}_{2} 1: 1\right) ;{ }^{1} \mathrm{H}-\mathrm{NMR}\left(400 \mathrm{MHz} ; \mathrm{CDCl}_{3} ; \mathrm{CHCl}_{3}=7.26 \mathrm{ppm}\right) \delta_{\mathrm{H}}=7.24-7.41(5 \mathrm{H}, \mathrm{m}, \mathrm{Ph})$, $6.56\left(1 \mathrm{H}, \mathrm{s}, \mathrm{H}_{1}\right), 6.13\left(1 \mathrm{H}, \mathrm{dd}, J=17.4,10.9 \mathrm{~Hz}, \mathrm{H}_{10}\right), 5.13\left(1 \mathrm{H}, \mathrm{dd}, J=17.4,1.4 \mathrm{~Hz}, \mathrm{H}_{11}\right), 4.86(1 \mathrm{H}, \mathrm{dd}, J$ $\left.=10.9,1.5 \mathrm{~Hz}, \mathrm{H}_{11}\right), 4.49-4.64\left(2 \mathrm{H}, \mathrm{m}, \mathrm{H}_{9}\right), 4.05-4.18\left(2 \mathrm{H}, \mathrm{m}, \mathrm{H}_{3}, \mathrm{H}_{7}\right), 3.73-3.83\left(1 \mathrm{H}, \mathrm{m}, \mathrm{H}_{5}\right), 2.18-2.36$ $\left(1 \mathrm{H}, \mathrm{m}, \mathrm{H}_{4}\right), 1.25\left(3 \mathrm{H}, \mathrm{d}, J=6.2 \mathrm{~Hz}, \mathrm{H}_{8}\right), 1.00\left(3 \mathrm{H}, \mathrm{d}, J=7.2 \mathrm{~Hz}, \mathrm{H}_{6}\right), 0.89(9 \mathrm{H}, \mathrm{s}, \mathrm{TBS}), 0.14(3 \mathrm{H}, \mathrm{s}, \mathrm{TBS})$, $0.07(3 \mathrm{H}, \mathrm{s}, \mathrm{TBS}) ;{ }^{13} \mathrm{C}-\mathrm{NMR}\left(101 \mathrm{MHz} ; \mathrm{CDCl}_{3} ; \mathrm{CDCl}_{3}=77.16 \mathrm{ppm}\right) \delta_{\mathrm{C}}=143.9$ (d, $\left.\mathrm{C}_{1}\right) 138.9$ (s, Ph), 134.2 (d, $\left.C_{10}\right), 128.4$ (d, Ph), 127.6 (d, Ph), 127.5 (d, Ph), 109.9 (t, $\left.C_{11}\right), 102.6$ (s, $\left.C_{2}\right), 81.3\left(d, C_{5}\right), 73.4,68.6$ $\left(d, C_{7}, C_{3}\right), 70.0\left(t, C_{9}\right), 35.9\left(d, C_{4}\right), 26.3$ (q, TBS), 18.6 (s, TBS), 16.9 (q, $C_{6}$ ), 15.3 (q, $C_{8}$ ), -2.9 (q, TBS), -4.0 (q, TBS); HRMS (ESI): $m / z$ calc. for $\mathrm{C}_{23} \mathrm{H}_{36} \mathrm{O}_{3} \mathrm{Si}[\mathrm{M}+\mathrm{Na}]^{+}:$: 411.2331, found 411.2321. 


\section{2-Nitroethyl acetate (SI-7)}<smiles>O=C(I)OCC[N+](=O)[O-]</smiles>

SI-7

Bismuth(III)trifuoromethane sulfonate $(0.43 \mathrm{~g}, 0.66 \mathrm{mmol}, 0.03 \mathrm{eq})$ was added to a solution of 2-nitroethanol (1.57 mL, $22.0 \mathrm{mmol}, 1.0 \mathrm{eq}$ ) and acetanhydride ( $3.10 \mathrm{~mL}, 33.0 \mathrm{mmol}, 1.5 \mathrm{eq}$ ) in $\mathrm{CH}_{2} \mathrm{Cl}_{2}$ $(60 \mathrm{~mL})$ at $0{ }^{\circ} \mathrm{C}$. The mixture was stirred for $2 \mathrm{~h}$ at $\mathrm{rt}$, then $\mathrm{MeOH}(20 \mathrm{~mL})$ was added and stirring was continued for $30 \mathrm{~min}$. Filtration through a pad of Celite ${ }^{\circledR}$ and removal of the solvent gave a residue which was dissolved in EtOAc and washed with a sat. aq. $\mathrm{NaHCO}_{3}$ solution and brine. The organic layer was dried over $\mathrm{MgSO}_{4}$, filtered and evaporated under reduced pressure. The crude product SI-7 ( $2.38 \mathrm{~g}, 17.8 \mathrm{mmol}, 81 \%$ ) was employed in the next step without further purification.

${ }^{1} \mathrm{H}-\mathrm{NMR}\left(200 \mathrm{MHz} ; \mathrm{CDCl}_{3} ; \mathrm{CHCl}_{3}=7.26 \mathrm{ppm}\right) \delta_{\mathrm{H}}=4.65\left(4 \mathrm{H}, \mathrm{s}, \mathrm{H}_{2}, \mathrm{H}_{3}\right), 2.13\left(3 \mathrm{H}, \mathrm{s}, \mathrm{H}_{1}\right)$.

The analytical data match those reported in the literature. ${ }^{4}$ 


\section{(E)-(2-Nitrovinyl)(phenyl)sulfane (SI-8)}<smiles>C/C(=C\C=[N+]([O-])[O-])Sc1ccccc1</smiles>

SI-8

Acetate SI-7 (2.38 g, $17.8 \mathrm{mmol}, 1.0 \mathrm{eq})$ and thiophenol $(1.83 \mathrm{~mL}, 17.8 \mathrm{mmol}, 1.0 \mathrm{eq})$ were dissolved in $\mathrm{MeCN}(50 \mathrm{~mL})$ and cooled to $0{ }^{\circ} \mathrm{C}$. To this solution was slowly added a solution of triethylamine (2.52 mL, $18.1 \mathrm{mmol}, 1.01 \mathrm{eq})$ in $\mathrm{MeCN}(10 \mathrm{~mL})$. The mixture was stirred for $40 \mathrm{~min}$ at $0{ }^{\circ} \mathrm{C}$, then poured into $100 \mathrm{~mL}$ of $1 \mathrm{M} \mathrm{HCl}$ and extracted with EtOAc. The organic layer was dried over $\mathrm{MgSO}_{4}$, filtered and evaporated under reduced pressure. The crude product $(3.28 \mathrm{~g}, 17.8 \mathrm{mmol}, 100 \%)$ was dissolved in $\mathrm{CH}_{2} \mathrm{Cl}_{2}(20 \mathrm{ml})$ and sulfuryl chloride $(1.54 \mathrm{~mL}, 19.0 \mathrm{mmol}, 1.06 \mathrm{eq})$ was added dropwise at $0{ }^{\circ} \mathrm{C}$. The mixture was stirred for $5 \mathrm{~min}$ at $0{ }^{\circ} \mathrm{C}$ after which time the volatile components were removed under reduced pressure. The residue was dissolved in $\mathrm{CH}_{2} \mathrm{Cl}_{2}(20 \mathrm{~mL})$ and cooled to $0{ }^{\circ} \mathrm{C}$. To this solution was added triethylamine $(2.65 \mathrm{~mL}, 19.0 \mathrm{mmol}, 1.06 \mathrm{eq})$ and the mixture was stirred for $30 \mathrm{~min}$ at $0^{\circ} \mathrm{C}$ and poured into water. Layers were separated and the organic layer was washed with $0.1 \mathrm{M} \mathrm{HCl}$ and dried over $\mathrm{MgSO}_{4}$. After filtration and removal of the solvent under reduced pressure the crude product was purified by column chromatography (PE-EtOAc= 20:1 $\rightarrow 5: 1$ ) to afford alkene SI-8 (1.62 g, $9.0 \mathrm{mmol}, 50 \%$ ) as a yellow oil.

${ }^{1} \mathrm{H}-\mathrm{NMR}\left(200 \mathrm{MHz} ; \mathrm{CDCl}_{3} ; \mathrm{CHCl}_{3}=7.26 \mathrm{ppm}\right) \delta_{\mathrm{H}}=8.31\left(1 \mathrm{H}, \mathrm{d}, J=12.7 \mathrm{~Hz}, \mathrm{H}_{1}\right), 7.46-7.64(5 \mathrm{H}, \mathrm{m}, \mathrm{Ph})$, $6.74\left(1 \mathrm{H}, \mathrm{d}, J=12.7 \mathrm{~Hz}, \mathrm{H}_{2}\right)$.

The analytical data match those reported in the literature. ${ }^{5}$ 


\section{(E)-(2-Nitrovinylsulfinyl)benzene (23)}<smiles>O=[N+]([O-])/C=C/[Sb]([O-])Oc1ccccc1</smiles>

23

Nitroolefin SI-8 (1.62 g, $9.0 \mathrm{mmol}, 1.0 \mathrm{eq})$ and meta-chloroperbenzoic acid (1.46 g, $8.5 \mathrm{mmol}$, 0.95 eq) were dissolved in $\mathrm{CH}_{2} \mathrm{Cl}_{2}(100 \mathrm{~mL})$ at $0{ }^{\circ} \mathrm{C}$. The mixture was stirred for $3.5 \mathrm{~h}$ at $0{ }^{\circ} \mathrm{C}$ and then water was added. The layers were separated and the organic physer was washed with a 0.1M NaOAc solution, with brine, and dried over $\mathrm{MgSO}_{4}$. After fltration and removal of the solvent under reduced pressure the crude product was recrystallized from EtOH to afford sulfoxide $23(0.718 \mathrm{~g}, 3.7 \mathrm{mmol}$, $41 \%)$ as a yellow solid.

m.p. $=89-93^{\circ} \mathrm{C}$ (lit. $\left.100-115{ }^{\circ} \mathrm{C}\right) ;{ }^{1} \mathrm{H}-\mathrm{NMR}\left(200 \mathrm{MHz} ; \mathrm{CDCl}_{3} ; \mathrm{CHCl}_{3}=7.26 \mathrm{ppm}\right) \delta_{\mathrm{H}}=7.36-8.11(7 \mathrm{H}, \mathrm{m})$. The analytical data match those reported in the literature. ${ }^{5}$ 
((2R,3S,4R)-2-((S)-1-(Benzyloxy)ethyl)-4-(tert-butyldimethylsilyloxy)-3-methyl-8-nitro-3,4,6,8atetrahydro-2H-chromen (25)
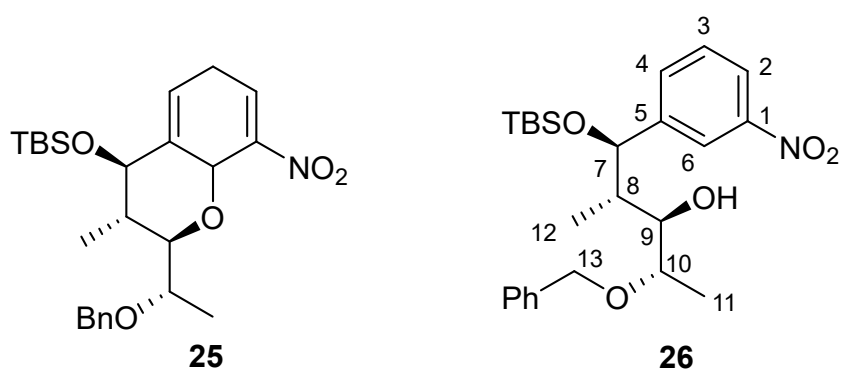

A solution of diene $22(15 \mathrm{mg}, 0.04 \mathrm{mmol}, 1.0 \mathrm{eq})$ and sulfoxide $23(8 \mathrm{mg}, 0.04 \mathrm{mmol}, 1.0 \mathrm{eq})$ in toluene $(1 \mathrm{~mL})$ was stirred for $1 \mathrm{~h}$ at $60^{\circ} \mathrm{C}$. After removal of the solvent under reduced pressure the residue was purified by column chromatography $\left(\mathrm{PE}-\mathrm{CH}_{2} \mathrm{Cl}_{2}=10: 1 \rightarrow 5: 1\right)$ to afford an undesired aromatic product 26 (12 $\mathrm{mg}, 0.027 \mathrm{mmol}, 67 \%)$.

${ }^{1} \mathrm{H}-\mathrm{NMR}\left(400 \mathrm{MHz} ; \mathrm{CDCl}_{3} ; \mathrm{CHCl}_{3}=7.26 \mathrm{ppm}\right) \delta_{\mathrm{H}}=8.23\left(1 \mathrm{H}, \mathrm{s}, \mathrm{H}_{6}\right), 8.09\left(1 \mathrm{H}, \mathrm{d}, J=7.9 \mathrm{~Hz}, \mathrm{H}_{2}\right), 7.65(1 \mathrm{H}$, $\left.\mathrm{d}, J=7.9 \mathrm{~Hz}, \mathrm{H}_{4}\right), 7.48\left(1 \mathrm{H}, \mathrm{t}, J=8.0, \mathrm{H}_{3}\right), 7.30-7.41(5 \mathrm{H}, \mathrm{m}, \mathrm{Ph}), 5.41\left(1 \mathrm{H}, \mathrm{s}, \mathrm{H}_{7}\right), 4.52-4.64\left(2 \mathrm{H}, \mathrm{m}, \mathrm{H}_{13}\right)$, $3.89\left(1 \mathrm{H}, \mathrm{d}, J=9.6 \mathrm{~Hz}, \mathrm{H}_{9}\right), 3.63\left(1 \mathrm{H}, \mathrm{dq}, J=6.3,3.0 \mathrm{~Hz}, \mathrm{H}_{10}\right), 2.53(1 \mathrm{H}, \mathrm{s}, \mathrm{OH}), 1.51-1.61\left(1 \mathrm{H}, \mathrm{m}, \mathrm{H}_{8}\right)$, $1.16\left(3 \mathrm{H}, \mathrm{d}, J=6.5 \mathrm{~Hz}, \mathrm{H}_{11}\right), 1.00(9 \mathrm{H}, \mathrm{s}, \mathrm{TBS}), 0.59\left(3 \mathrm{H}, \mathrm{d}, J=6.8 \mathrm{~Hz}, \mathrm{H}_{12}\right), 0.13(3 \mathrm{H}, \mathrm{s}, \mathrm{TBS}),-0.15(3 \mathrm{H}$, $\mathrm{s}, \mathrm{TBS}) ; \mathrm{HRMS}$ (ESI): $\mathrm{m} / z$ calc. for $\mathrm{C}_{25} \mathrm{H}_{37} \mathrm{NO}_{5} \mathrm{Si}[\mathrm{M}+\mathrm{Na}]^{+}:$482.2339, found 482.2332. 
(2R,3S,4S)-2-((S)-1-(Hydroxy)ethyl)-4-(tert-butyldimethylsilyloxy)-3-methyl-3,4-dihydro-2H-pyran (SI-9)

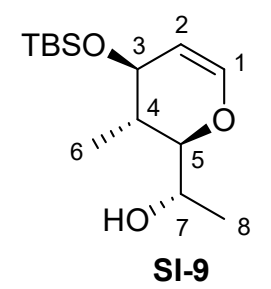

To a solution of benzyl ether $20(630 \mathrm{mg}, 1.74 \mathrm{mmol}, 1.0 \mathrm{eq})$ in dry THF (10 mL) was added lithium ditertbutylbiphenyl $1 \mathrm{M}$ solution in THF $(15 \mathrm{ml}, 15 \mathrm{mmol}, 7.5 \mathrm{eq})$ dropwise at $-78{ }^{\circ} \mathrm{C}$. The reaction was terminated by addition of a sat. aq. $\mathrm{NH}_{4} \mathrm{Cl}$ solution, layers were separated and the aqueous layer was extracted with EtOAc. The combined organic phases were dried over $\mathrm{MgSO}_{4}$, filtered and evaporated under reduced pressure. The crude product was purified by column chromatography (PE-EtOAc= 20:1 $\rightarrow 10: 1$ ) to afford alcohol SI-9 (440 mg, $1.62 \mathrm{mmol}, 93 \%$ ) as a colorless oil.

$\mathbf{R}_{\mathbf{f}}=0.29\left(\right.$ PE-EtOAC 20:1); $[\alpha]^{21}{ }_{\mathrm{D}}=+88.1^{\circ}\left(c=1.00 ; \mathrm{CHCl}_{3}\right) ;{ }^{1} \mathrm{H}-\mathrm{NMR}\left(400 \mathrm{MHz} ; \mathrm{CDCl}_{3} ; \mathrm{CHCl}_{3}=7.26\right.$ ppm) $\delta_{H}=6.34\left(1 \mathrm{H}, \mathrm{d}, J=6.4 \mathrm{~Hz}, \mathrm{H}_{1}\right), 4.70-4.76\left(1 \mathrm{H}, \mathrm{m}, \mathrm{H}_{2}\right), 3.99\left(1 \mathrm{H}, \mathrm{se}, J=6.4 \mathrm{~Hz}, \mathrm{H}_{7}\right), 3.78-3.85(1 \mathrm{H}$, $\left.\mathrm{m}, \mathrm{H}_{3}\right), 3.66\left(1 \mathrm{H}, \mathrm{dd}, J=6.8,4.3 \mathrm{~Hz}, \mathrm{H}_{5}\right), 3.05(1 \mathrm{H}, \mathrm{d}, J=6.0 \mathrm{~Hz}, \mathrm{OH}), 2.08-2.23\left(1 \mathrm{H}, \mathrm{m}, \mathrm{H}_{4}\right), 1.26(3 \mathrm{H}, \mathrm{d}$, $\left.J=6.3 \mathrm{~Hz}, \mathrm{H}_{8}\right), 0.98\left(3 \mathrm{H}, \mathrm{d}, J=7.2 \mathrm{~Hz}, \mathrm{H}_{6}\right), 0.92(9 \mathrm{H}, \mathrm{s}, \mathrm{TBS}), 0.12(6 \mathrm{H}, \mathrm{s}, \mathrm{TBS}) ;{ }^{13} \mathrm{C}-\mathrm{NMR}(101 \mathrm{MHz}$; $\left.\mathrm{CDCl}_{3} ; \mathrm{CDCl}_{3}=77.16 \mathrm{ppm}\right) \delta_{\mathrm{C}}=143.7\left(\mathrm{~d}, \mathrm{C}_{1}\right), 102.5\left(\mathrm{~d}, \mathrm{C}_{2}\right), 82.7\left(\mathrm{~d}, \mathrm{C}_{5}\right), 66.8\left(\mathrm{~d}, \mathrm{C}_{3}\right), 66.1\left(\mathrm{~d}, \mathrm{C}_{7}\right), 34.8$ (d, $\mathrm{C}_{4}$ ), 26.0 (q, TBS), 19.4 (s, TBS), 18.2 (q, $\mathrm{C}_{6}$ ), 15.8 (q, $\mathrm{C}_{8}$ ), -4.1 (q, TBS), -4.7 (q, TBS); HRMS (ESI): m/z calc. for $\mathrm{C}_{14} \mathrm{H}_{28} \mathrm{O}_{3} \mathrm{Si}[\mathrm{M}+\mathrm{Na}]^{+}:$295.1705, found 295.1707. 
Tert-butyl(((2R,3S,4S)-2-((S)-1-(methoxymethoxy)ethyl)-3-methyl-3,4-dihydro-2H-pyran-4yl)oxy)dimethylsilane (27)<smiles>CC(C)[C@H]1C=CO[C@H](C(C)C)[C@@H]1O</smiles>

27

To a solution of alcohol SI-9 (2.77 g, $10 \mathrm{mmol}, 1,0 \mathrm{eq})$ in diisopropylethylamine $(12 \mathrm{ml})$ was added chloromethyl methyl ether $(1.3 \mathrm{ml}, 16 \mathrm{mmol}, 1.6 \mathrm{eq})$ at $0{ }^{\circ} \mathrm{C}$. The reaction was stirred overnight at $\mathrm{rt}$ and then diluted with $\mathrm{CH}_{2} \mathrm{Cl}_{2}$, washed witha sat. aq. $\mathrm{NH}_{4} \mathrm{Cl}$ solution (2x). The organic phases were dried over $\mathrm{MgSO}_{4}$, filtered and evaporated under reduced pressure. The crude product was purified by column chromatography (PE-EtOAc= 30:1) to afford dihydropyran $27(3.08 \mathrm{~g}, 9.7 \mathrm{mmol}, 97 \%)$ as a colorless oil.

$\mathbf{R}_{\mathbf{f}}=0.52\left(\right.$ PE-EtOAC 5:1); $[\alpha]^{21}{ }_{\mathrm{D}}=+73.8^{\circ}\left(c=1.00 ; \mathrm{CHCl}_{3}\right) ;{ }^{1} \mathrm{H}-\mathrm{NMR}\left(400 \mathrm{MHz} ; \mathrm{CDCl}_{3} ; \mathrm{CHCl}_{3}=7.26 \mathrm{ppm}\right)$ $\delta_{\mathrm{H}}=6.40\left(1 \mathrm{H}, \mathrm{dd}, J=6.2,1.2 \mathrm{~Hz}, \mathrm{H}_{1}\right), 4.72-4.71(2 \mathrm{H}, \mathrm{m}, \mathrm{OMOM}), 4.65\left(1 \mathrm{H}, \mathrm{dd}, J=6.2,2.0 \mathrm{~Hz}, \mathrm{H}_{2}\right)$, 4.00-3.95 (2H, m, $\left.\mathrm{H}_{3}, \mathrm{H}_{6}\right), 3.83\left(1 \mathrm{H}, \mathrm{dd}, J=10.3,2.9 \mathrm{~Hz}, \mathrm{H}_{5}\right), 3.38(3 \mathrm{H}, \mathrm{s}, \mathrm{OMOM}), 1.77-1.68(1 \mathrm{H}, \mathrm{m}$, $\left.\mathrm{H}_{4}\right), 1.21\left(3 \mathrm{H}, \mathrm{d}, J=6.4 \mathrm{~Hz}, \mathrm{H}_{8}\right), 0.97\left(3 \mathrm{H}, \mathrm{d}, J=6.8 \mathrm{~Hz}, \mathrm{H}_{6}\right), 0.91(9 \mathrm{H}, \mathrm{s}, \mathrm{TBS}), 0.09(3 \mathrm{H}, \mathrm{s}, \mathrm{TBS}), 0.08(3 \mathrm{H}$ s, TBS); ${ }^{13} \mathrm{C}-\mathrm{NMR}\left(101 \mathrm{MHz} ; \mathrm{CDCl}_{3} ; \mathrm{CDCl}_{3}=77.16 \mathrm{ppm}\right) \delta_{\mathrm{C}}=144.1\left(\mathrm{~d}, \mathrm{C}_{1}\right), 105.5\left(\mathrm{~d}, \mathrm{C}_{2}\right), 95.2$ (t, OMOM), 81.0 (d, $C_{5}$ ), 72.1 (d, $C_{7}$ ), 69.7 (d, $C_{3}$ ), 55.5 (q, OMOM), 37.4 (d, $C_{4}$ ), 26.0 (q, TBS), 18.2 (s, TBS), 14.1 (q, $\mathrm{C}_{6}$ ), 13.4 (q, $\mathrm{C}_{8}$ ), -4.0 (q, TBS), -4.5 (q, TBS); HRMS (ESI): $\mathrm{m} / z$ calc. for $\mathrm{C}_{16} \mathrm{H}_{32} \mathrm{O}_{4} \mathrm{Si}[\mathrm{M}+\mathrm{Na}]^{+}$: 339.1966, found 339.1965. 
tert-Butyl(((2R,3S,4R)-2-((S)-1-(methoxymethoxy)ethyl)-3-methyl-6-vinyl-3,4-dihydro-2H-pyran-4yl)oxy)dimethylsilane (28)

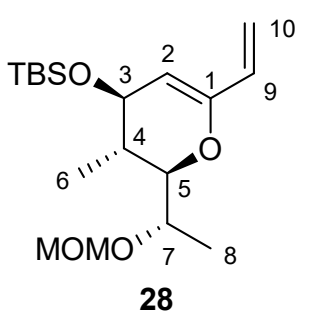

To a solution of enol ether 27 (316 mg, $0.95 \mathrm{mmol}, 1.0 \mathrm{eq})$ in THF ( $6 \mathrm{ml}$ ) was added s-BuLi solution $1.3 \mathrm{M}$ in hexane $(2.4 \mathrm{ml}, 3.12 \mathrm{mmol}, 3.3 \mathrm{eq})$ dropwise at $-78{ }^{\circ} \mathrm{C}$. After stirring at $\mathrm{rt}$ for $10 \mathrm{~min}$ the solution was again cooled to $-78{ }^{\circ} \mathrm{C}$ and a solution of diiodomethane $(961 \mathrm{mg}, 3.42 \mathrm{mmol}, 3.6 \mathrm{eq})$ in THF (6 ml) was added dropwise. The reaction mixture was warmed to rt and stirring was continued for $30 \mathrm{~min}$. The reaction was terminated by addition of a sat. aq. $\mathrm{Na}_{2} \mathrm{~S}_{2} \mathrm{O}_{3}$ solution, layers were separated and the aqueous layer was extracted with EtOAc. The combined organic phases were dried over $\mathrm{MgSO}_{4}$, filtered and evaporated under reduced pressure. The crude product was dissolved in degassed THF ( $5 \mathrm{ml}$ ) and Cul ( $38 \mathrm{mg}, 0.20 \mathrm{mmol}, 0.2$ eq), PEPPSIiPr ( $68 \mathrm{mg}, 0.10 \mathrm{mmol}, 0.1 \mathrm{eq}$ ) and CsF $(570 \mathrm{mg}, 3.8 \mathrm{mmol}, 4 \mathrm{eq}$ ) were added and the resulting mixture was stirred for $5 \mathrm{~min}$. Tributyl(vinyl)tin $(0.42 \mathrm{ml}, 1.42 \mathrm{mmol}, 1.5 \mathrm{eq})$ were added dropwise and the reaction was stirred for $12 \mathrm{~h}$ at rt. Filtration over Celite $^{\circledR}$, evaporation of the volatiles and column chromatography (PEEtOAc $=50: 1$ ) afforded diene 28 (304 mg, $0.89 \mathrm{mmol}, 93 \%$ ) as a colorless oil.

$\mathbf{R}_{\mathbf{f}}=0.50\left(\right.$ PE-EtOAc 10:1); $[\alpha]^{26}{ }_{\mathrm{D}}=+57.0^{\circ}\left(c=1.00 ; \mathrm{CH}_{2} \mathrm{Cl}_{2}\right) ;{ }^{1} \mathrm{H}-\mathrm{NMR}\left(400 \mathrm{MHz} ; \mathrm{C}_{6} \mathrm{D}_{6} ; \mathrm{C}_{6} \mathrm{D}_{5} \mathrm{H}=7.16\right.$ ppm) $\delta_{H}=6.01\left(1 \mathrm{H}, \mathrm{dd}, J=17.1,10.4 \mathrm{~Hz}, \mathrm{H}_{9}\right), 5.89\left(1 \mathrm{H}, \mathrm{dd}, J=17.1,2.2 \mathrm{~Hz}, \mathrm{H}_{10}\right), 5.04(1 \mathrm{H}, \mathrm{dd}, J=10.4$, $\left.2.2 \mathrm{~Hz}, \mathrm{H}_{2}\right), 4.82\left(1 \mathrm{H}, \mathrm{d}, J=2.8 \mathrm{~Hz}, \mathrm{H}_{10^{\prime}}\right), 4.70(1 \mathrm{H}, \mathrm{d}, J=6.8 \mathrm{~Hz}, \mathrm{OMOM}), 4.59(1 \mathrm{H}, \mathrm{d}, J=6.8 \mathrm{~Hz}$, OMOM), $4.04\left(1 \mathrm{H}, \mathrm{qd}, J=6.4,4.0 \mathrm{~Hz}, \mathrm{H}_{7}\right), 3.93\left(1 \mathrm{H}, \mathrm{dd}, J=6.9,2.8, \mathrm{H}_{3}\right), 3.89(1 \mathrm{H}, \mathrm{dd}, J=8.6,4.0 \mathrm{~Hz}$, $\mathrm{H}_{5}$ ), $3.22(3 \mathrm{H}, \mathrm{s}, \mathrm{OMOM}), 2.03-1.91\left(1 \mathrm{H}, \mathrm{m}, \mathrm{H}_{4}\right), 1.25\left(3 \mathrm{H}, \mathrm{d}, J=6.4 \mathrm{~Hz}, \mathrm{H}_{8}\right), 1.02-0.94(9 \mathrm{H}, \mathrm{m}, \mathrm{TBS})$, $0.86\left(3 \mathrm{H}, \mathrm{d}, J=6.9 \mathrm{~Hz}, \mathrm{H}_{6}\right), 0.09(3 \mathrm{H}, \mathrm{s}, \mathrm{TBS}), 0.07(3 \mathrm{H}, \mathrm{s}, \mathrm{TBS}) ;{ }^{13} \mathrm{C}-\mathrm{NMR}\left(101 \mathrm{MHz} ; \mathrm{C}_{6} \mathrm{D}_{6} ; \mathrm{C}_{6} \mathrm{D}_{5} \mathrm{H}=\right.$ $128.06 \mathrm{ppm}) \delta_{\mathrm{C}}=150.9\left(\mathrm{~s}, \mathrm{C}_{1}\right), 132.5\left(\mathrm{~d}, \mathrm{C}_{9}\right), 114.7\left(\mathrm{t}, \mathrm{C}_{10}\right), 106.2\left(\mathrm{~d}, \mathrm{C}_{2}\right), 95.9(\mathrm{t}, \mathrm{OMOM}), 81.3\left(\mathrm{~d}, \mathrm{C}_{5}\right)$, $73.3\left(d, C_{7}\right), 70.6\left(d, C_{3}\right), 55.1$ (q, OMOM), 37.2 (d, $C_{4}$ ), 26.1 (q, TBS), 18.4 (s, TBS), 15.6 (q, $C_{8}$ ), 14.7 (q, $\mathrm{C}_{6}$ ), -3.98 (q, TBS), -4.42 (q, TBS); HRMS (ESI): $\mathrm{m} / \mathrm{z}$ calc. for $\mathrm{C}_{18} \mathrm{H}_{34} \mathrm{O}_{4} \mathrm{Si}[\mathrm{M}+\mathrm{Na}]^{+}: 365.2124$, found 365.2133 . 


\section{1-((2R,3S,4R)-4-((tert-Butyldimethylsilyl)oxy)-2-((S)-1-(methoxymethoxy)ethyl)-3-methylchroman-}

\section{6-yl)ethan-1-one (31)}<smiles>CC(=O)c1ccc2c(c1)[C@@H](OC(C)C)[C@H](C)[C@@H](C(C)C)O2</smiles>

A solution of diene 28 ( $210 \mathrm{mg}, 0.61 \mathrm{mmol}, 1.0 \mathrm{eq})$ and 3-butyn-2-one (47 $\mu \mathrm{l}, 0.61 \mathrm{mmol}, 1 \mathrm{eq})$ in benzene $(3 \mathrm{ml})$ were pressurized to $14 \mathrm{kbar}$ for $15 \mathrm{~h}$. After evaporation of the volatiles the crude product was purified by column chromatography (PE-EtOAc= 50:1 $\rightarrow$ 20:1 $\rightarrow$ 10:1) to afford the title compound ( $85 \mathrm{mg}, 0.21 \mathrm{mmol}, 35 \%$ ) along with diene 28 (107 mg, $0.31 \mathrm{mmol}, 51 \%$ ). The Diels-Alder cycloaddition product ( $116 \mathrm{mg}, 0.28 \mathrm{mmol}, 1.0 \mathrm{eq}$ ) was dissolved in $\mathrm{CH}_{2} \mathrm{Cl}_{2}(3 \mathrm{ml})$ and cooled to $0^{\circ} \mathrm{C}$. After dropwise addition of a solution of DDQ (64 mg, $0.28 \mathrm{mmol}, 1.0 \mathrm{eq}$ ) in $\mathrm{CH}_{2} \mathrm{Cl}_{2}$ (3 ml) the reaction was stirred for $30 \mathrm{~min}$ and then terminated by a addition of a sat. aq. $\mathrm{NH}_{4} \mathrm{Cl}$ solution. Layers were separated and the aqueous phase was extracted with $\mathrm{CH}_{2} \mathrm{Cl}_{2}$. The combined organic phases were dried over $\mathrm{MgSO}_{4}$, filtered and evaporated under reduced pressure. The crude product was purified by column chromatography (PE-EtOAc= 10:1) to afford chromane $31(80 \mathrm{mg}, 0.20 \mathrm{mmol}, 70 \%)$ as a colorless oil.

$\mathbf{R}_{\mathbf{f}}=0.22\left(\right.$ PE-EtOAC 5:1); $[\alpha]^{26}{ }_{\mathrm{D}}=-37.0^{\circ}\left(c=1.00 ; \mathrm{CH}_{2} \mathrm{Cl}_{2}\right) ;{ }^{1} \mathrm{H}-\mathrm{NMR}\left(400 \mathrm{MHz} ; \mathrm{C}_{6} \mathrm{D}_{6} ; \mathrm{C}_{6} \mathrm{D}_{5} \mathrm{H}=7.16 \mathrm{ppm}\right)$ $\delta_{\mathrm{H}}=8.24\left(1 \mathrm{H}, \mathrm{d}, J=2.0 \mathrm{~Hz}, \mathrm{H}_{8}\right), 7.64\left(1 \mathrm{H}, \mathrm{dd}, J=8.5,2.0 \mathrm{~Hz}, \mathrm{H}_{10}\right), 6.86\left(1 \mathrm{H}, \mathrm{d}, J=8.5 \mathrm{~Hz}, \mathrm{H}_{11}\right), 4.65(1 \mathrm{H}$, $\mathrm{d}, J=6.9 \mathrm{~Hz}, \mathrm{OMOM}), 4.59(1 \mathrm{H}, \mathrm{d}, J=6.9 \mathrm{~Hz}, \mathrm{OMOM}), 4.26\left(1 \mathrm{H}, \mathrm{d}, J=8.5 \mathrm{~Hz}, \mathrm{H}_{6}\right), 4.03(1 \mathrm{H}, \mathrm{dd}, J=9.1$, $\left.3.4 \mathrm{~Hz}, \mathrm{H}_{3}\right), 3.96\left(1 \mathrm{H}, \mathrm{qd}, J=6.4,3.4 \mathrm{~Hz}, \mathrm{H}_{2}\right), 3.22(3 \mathrm{H}, \mathrm{s}, \mathrm{OMOM}), 2.21\left(3 \mathrm{H}, \mathrm{s}, \mathrm{H}_{14}\right), 2.00-1.86(1 \mathrm{H}, \mathrm{m}$, $\left.\mathrm{H}_{8}\right), 1.19\left(3 \mathrm{H}, \mathrm{d}, J=6.4 \mathrm{~Hz}, \mathrm{H}_{1}\right), 1.04(9 \mathrm{H}, \mathrm{s}, \mathrm{TBS}), 0.85\left(3 \mathrm{H}, \mathrm{d}, J=6.8 \mathrm{~Hz}, \mathrm{H}_{5}\right) ;{ }^{13} \mathrm{C}-\mathrm{NMR}\left(101 \mathrm{MHz} ; \mathrm{C}_{6} \mathrm{D}_{6}\right.$; $\left.\mathrm{C}_{6} \mathrm{D}_{5} \mathrm{H}=128.06 \mathrm{ppm}\right) \delta_{\mathrm{C}}=195.0\left(\mathrm{~s}, \mathrm{C}_{13}\right), 158.4\left(\mathrm{~s}, \mathrm{C}_{12}\right), 130.8\left(\mathrm{~s}, \mathrm{C}_{9}\right), 129.9\left(\mathrm{~d}, \mathrm{C}_{10}\right), 128.9\left(\mathrm{~d}, \mathrm{C}_{8}\right), 126.4$ $\left(\mathrm{s}, \mathrm{C}_{10}\right), 116.6\left(\mathrm{~d}, \mathrm{C}_{14}\right), 95.8$ (t, OMOM), 82.4 (d, $\mathrm{C}_{7}$ ), 73.7 (d, $\mathrm{C}_{6}$ ), 71.5 (d, $\left.\mathrm{C}_{9}\right), 55.2$ (q, OMOM), 37.2 (d, $C_{8}$ ), 26.2 ( $q, T B S$ ), 26.1 ( $q, C_{21}$ ), 18.5 (s, TBS), 14.8 (q, $C_{18}$ ), 14.6 ( $\left.q, C_{19}\right),-3.6$ (q, TBS), -3.64 (q, TBS); HRMS (ESI): $m / z$ calc. for $\mathrm{C}_{22} \mathrm{H}_{36} \mathrm{O}_{5} \mathrm{Si}[\mathrm{M}+\mathrm{Na}]^{+}: 431.2230$, found 431.2229 . 
Diels-Alder products (exo-35a) and (endo-35b)

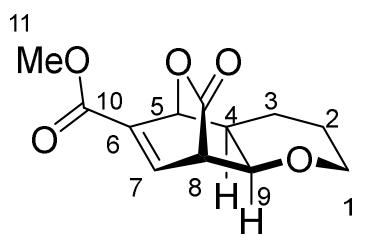

exo-35a

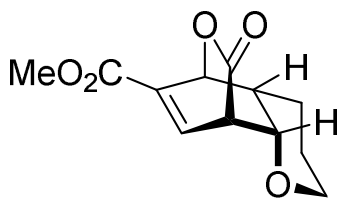

endo-35b

A solution of 3,4-dihydro-2H-pyran (166 $\mu \mathrm{l}, 1.83 \mathrm{mmol}, 1.0 \mathrm{eq})$ and methyl 2-pyrone-5-carboxylate (423 mg, $2.75 \mathrm{mmol}, 1.5 \mathrm{eq}$ ) in $\mathrm{CH}_{2} \mathrm{Cl}_{2}(4.6 \mathrm{ml}$ ) was pressurized to $14 \mathrm{kbar}$ for $7 \mathrm{~d}$. After evaporation of the volatiles the crude product was purified by column chromatography (PE-EtOAc= 5:1 $\rightarrow 1: 1$ ) to afford the cycloaddition products 35 (365 mg, $1.54 \mathrm{mmol}, 84 \%$, endo:exo 1:1.8) as racemic mixtures.

exo-35a: $\mathbf{R}_{\mathbf{f}}=0.12$ (PE-EtOAc 1:1); m.p. = 141.8-143.4 ${ }^{\circ} \mathrm{C}$; ${ }^{1} \mathrm{H}-\mathrm{NMR}\left(400 \mathrm{MHz} ; \mathrm{CDCl}_{3} ; \mathrm{CHCl}_{3}=7.26\right.$ ppm) $\delta_{H}=7.17\left(1 \mathrm{H}, \mathrm{dd}, J=6.7,2.2 \mathrm{~Hz}, \mathrm{H}_{7}\right), 5.39-5.38\left(1 \mathrm{H}, \mathrm{m}, \mathrm{H}_{5}\right), 3.95(1 \mathrm{H}, \mathrm{ddd}, J=10.8,10.0,7.8 \mathrm{~Hz}$, $\left.\mathrm{H}_{1}\right), 3.82\left(1 \mathrm{H}, \mathrm{dd}, J=6.6,3.3 \mathrm{~Hz}, \mathrm{H}_{8}\right), 3.79\left(3 \mathrm{H}, \mathrm{s}, \mathrm{H}_{11}\right), 3.66\left(1 \mathrm{H}, \mathrm{dd}, J=8.1,3.2 \mathrm{~Hz}, \mathrm{H}_{9}\right), 3.58(1 \mathrm{H}, \mathrm{ddd}, J$ $\left.=11.3,9.3,4.0 \mathrm{~Hz}, \mathrm{H}_{1^{\prime}}\right), 1.96-1.82\left(2 \mathrm{H}, \mathrm{m}, \mathrm{H}_{2}, \mathrm{H}_{4}\right), 1.80-1.65\left(2 \mathrm{H}, \mathrm{m}, \mathrm{H}_{3}\right), 1.57-1.45\left(1 \mathrm{H}, \mathrm{m}, \mathrm{H}_{2^{\prime}}\right) ;{ }^{13} \mathrm{C}-$ NMR (101 MHz; $\left.\mathrm{CDCl}_{3} ; \mathrm{CDCl}_{3}=77.16 \mathrm{ppm}\right) \delta_{\mathrm{c}}=169.8\left(\mathrm{~s}, \mathrm{C}_{12}\right), 162.6\left(\mathrm{~s}, \mathrm{C}_{10}\right), 138.4\left(\mathrm{~s}, \mathrm{C}_{6}\right), 136.9\left(\mathrm{~d}, \mathrm{C}_{7}\right)$, $76.7\left(d, C_{5}\right), 69.4\left(d, C_{9}\right), 64.0\left(t, C_{1}\right), 52.4\left(q, C_{11}\right), 48.4\left(d, C_{8}\right), 37.8\left(d, C_{4}\right), 20.8\left(t, C_{2}\right), 18.5\left(t, C_{3}\right)$.

endo-35b: $\mathbf{R}_{\mathbf{f}}=0.22$ (PE-EtOAc 1:1); m.p. $=119.6-120.7{ }^{\circ} \mathrm{C} ;{ }^{1} \mathbf{H}-\mathrm{NMR}\left(400 \mathrm{MHz} ; \mathrm{CDCl}_{3} ; \mathrm{CHCl}_{3}=7.26\right.$ ppm) $\delta_{H}=7.27\left(1 \mathrm{H}, \mathrm{ddd}, J=6.3,1.8,0.6 \mathrm{~Hz}, \mathrm{H}_{7}\right), 5.51\left(1 \mathrm{H}, \mathrm{dd}, J=3.2,2.0 \mathrm{~Hz}, \mathrm{H}_{5}\right), 3.98(1 \mathrm{H}, \mathrm{dd}, J=6.2$, $\left.3.7 \mathrm{~Hz}, \mathrm{H}_{8}\right), 3.94\left(1 \mathrm{H}, \mathrm{dd}, J=7.2,3.7 \mathrm{~Hz}, \mathrm{H}_{9}\right), 3.81\left(3 \mathrm{H}, \mathrm{s}, \mathrm{H}_{11}\right), 3.77-3.70\left(1 \mathrm{H}, \mathrm{m}, \mathrm{H}_{1}\right), 3.62-3.54(1 \mathrm{H}, \mathrm{m}$, $\left.\mathrm{H}_{1^{\prime}}\right), 2.41-2.34\left(1 \mathrm{H}, \mathrm{m}, \mathrm{H}_{4}\right), 1.83-1.73\left(2 \mathrm{H}, \mathrm{m}, \mathrm{H}_{2}, \mathrm{H}_{3}\right), 1.59-1.49\left(1 \mathrm{H}, \mathrm{m}, \mathrm{H}_{2^{\prime}}\right), 0.94-0.83\left(1 \mathrm{H}, \mathrm{m}, \mathrm{H}_{3^{\prime}}\right)$. 
Methyl 3,4,4a,8a-tetrahydro-2H-chromene-6-carboxylate (36)<smiles>COC(=O)C1=CC=C2CCCOC2C=C1</smiles>

A solution of cycloaddition adduct $35(441 \mathrm{mg}, 1.85 \mathrm{mmol}, 1.00 \mathrm{eq})$ in toluene (15 ml) was heated to $185{ }^{\circ} \mathrm{C}$ in a sealed tube over $3 \mathrm{~d}$. Evaporation of the solvent and purification by column chromatoraphy (PE-EtOAc 3:1) afforded cyclohexadiene 36 (258 mg, $1.33 \mathrm{mmol}, 72 \%$ ) as a colorless oil.

$\mathbf{R}_{\mathbf{f}}=0.40\left(\right.$ PE-EtOAC 5:1); ${ }^{1} \mathrm{H}-\mathrm{NMR}\left(400 \mathrm{MHz} ; \mathrm{CDCl}_{3} ; \mathrm{CHCl}_{3}=7.26 \mathrm{ppm}\right) \delta_{\mathrm{H}}=6.96-6.93\left(1 \mathrm{H}, \mathrm{m}, \mathrm{H}_{5}\right)$, $6.65\left(1 \mathrm{H}, \mathrm{dd}, J=9.7,1.5 \mathrm{~Hz}, \mathrm{H}_{7}\right), 6.03\left(1 \mathrm{H}, \mathrm{dd}, J=9.8,5.0 \mathrm{~Hz}, \mathrm{H}_{8}\right), 4.04\left(1 \mathrm{H}, \mathrm{t}, J=5.4 \mathrm{~Hz}, \mathrm{H}_{9}\right), 3.81-3.75$ $\left(1 \mathrm{H}, \mathrm{m}, \mathrm{H}_{1}\right), 3.78\left(3 \mathrm{H}, \mathrm{s}, \mathrm{H}_{11}\right), 3.46\left(1 \mathrm{H}, \mathrm{ddd}, J=11.3,10.0,2.6 \mathrm{~Hz}, \mathrm{H}_{1^{\prime}}\right), 2.48-2.43\left(1 \mathrm{H}, \mathrm{m}, \mathrm{H}_{4}\right), 1.99-$ $1.84\left(2 \mathrm{H}, \mathrm{m}, \mathrm{H}_{3}\right), 1.70-1.60\left(1 \mathrm{H}, \mathrm{m}, \mathrm{H}_{2}\right), 1.57-1.49\left(1 \mathrm{H}, \mathrm{m}, \mathrm{H}_{2^{\prime}}\right) ;{ }^{13} \mathrm{C}-\mathrm{NMR}\left(101 \mathrm{MHz} ; \mathrm{CDCl}_{3} ; \mathrm{CDCl}_{3}=\right.$ $77.16 \mathrm{ppm}) \delta_{c}=165.8\left(\mathrm{~s}, \mathrm{C}_{10}\right), 142.8\left(\mathrm{~d}, \mathrm{C}_{5}\right), 128.8\left(\mathrm{~s}, \mathrm{C}_{6}\right), 126.3\left(\mathrm{~d}, \mathrm{C}_{8}\right), 125.1\left(\mathrm{~d}, \mathrm{C}_{7}\right), 70.0\left(\mathrm{~d}, \mathrm{C}_{9}\right), 65.9$ $\left(t, C_{1}\right), 51.9\left(q, C_{11}\right), 35.9\left(d, C_{4}\right), 26.5\left(t, C_{3}\right), 23.6\left(t, C_{2}\right)$. 


\section{Methyl chromane-6-carboxylate (37)}

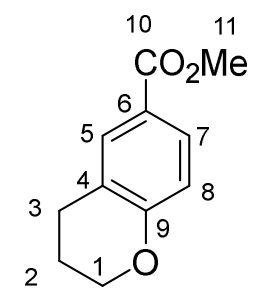

37

To a solution of hexadiene $36(40 \mathrm{mg}, 0.21 \mathrm{mmol}, 1.0 \mathrm{eq})$ in $\mathrm{CH}_{2} \mathrm{Cl}_{2}(1 \mathrm{ml})$ was added a solution of DDQ (94 mg, $0.41 \mathrm{mmol}, 2.0 \mathrm{eq}$ ) in $\mathrm{CH}_{2} \mathrm{Cl}_{2}(2 \mathrm{ml})$ dropwise at $0{ }^{\circ} \mathrm{C}$. After stirring for $12 \mathrm{~h}$ at $\mathrm{rt}$ the reaction was terminated by addition of a sat. aq. $\mathrm{Na}_{2} \mathrm{~S}_{2} \mathrm{O}_{3}$ solution followed by a sat. aq. $\mathrm{NaHCO}_{3}$ solution.Layers were separated and the aqueous phase was extracted with $\mathrm{CH}_{2} \mathrm{Cl}_{2}$. The combined organic phases were washed with $1 \mathrm{M}$ aq. $\mathrm{NaOH}$, water and brine, dried over $\mathrm{MgSO}_{4}$, filtered and evaporated under reduced pressure. The crude product was purified by column chromatography (PEEtOAc $=5: 1)$ to afford chromane $37(20 \mathrm{mg}, 0.10 \mathrm{mmol}, 50 \%)$ as a colorless oil.

$\mathbf{R}_{\mathbf{f}}=0.47\left(\right.$ PE-EtOAC 5:1); ${ }^{1} \mathrm{H}-\mathrm{NMR}\left(400 \mathrm{MHz} ; \mathrm{CDCl}_{3} ; \mathrm{CHCl}_{3}=7.26 \mathrm{ppm}\right) \delta_{\mathrm{H}}=7.77-7.74\left(2 \mathrm{H}, \mathrm{m}, \mathrm{H}_{5}, \mathrm{H}_{7}\right)$, $6.79\left(1 \mathrm{H}, \mathrm{d}, J=9.0 \mathrm{~Hz}, \mathrm{H}_{8}\right), 4.23\left(2 \mathrm{H}, \mathrm{t}, J=5.17 \mathrm{~Hz}, \mathrm{H}_{1}\right), 3.86\left(3 \mathrm{H}, \mathrm{s}, \mathrm{H}_{11}\right), 2.81\left(2 \mathrm{H}, \mathrm{t}, J=6.44 \mathrm{~Hz}, \mathrm{H}_{3}\right)$, 2.04-1.98 (2H, m, H2); ${ }^{13} \mathrm{C}-\mathrm{NMR}\left(101 \mathrm{MHz} ; \mathrm{CDCl}_{3} ; \mathrm{CDCl}_{3}=77.16 \mathrm{ppm}\right) \delta_{\mathrm{c}}=167.2\left(\mathrm{~s}, \mathrm{C}_{10}\right), 159.1\left(\mathrm{~s}, \mathrm{C}_{9}\right)$, $132.0\left(\mathrm{~d}, \mathrm{C}_{7}\right), 129.2\left(\mathrm{~d}, \mathrm{C}_{5}\right), 122.1\left(\mathrm{~s}, \mathrm{C}_{4}\right), 122.0\left(\mathrm{~s}, \mathrm{C}_{6}\right), 116.8\left(\mathrm{~d}, \mathrm{C}_{8}\right), 67.0$ (t, $\left.\mathrm{C}_{1}\right), 51.9$ (q, $\left.\mathrm{C}_{11}\right), 24.9$ (t, $\left.C_{3}\right), 22.1\left(t, C_{2}\right)$.

The analytical data match those reported in the literature. ${ }^{6}$ 
Methyl (3S,4R)-4-((tert-butyldimethylsilyl)oxy)-2-((S)-1-(methoxymethoxy)ethyl)-3-methyl-9-oxo3,4,4a,5,8,8a-hexahydro-2H-5,8-(epoxymethano)chromene-6-carboxylate (SI-10)

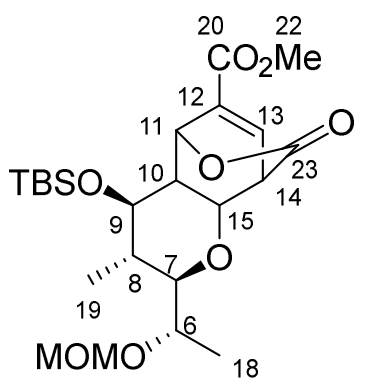

SI-10

A solution of dihydropyran $27(1.1 \mathrm{~g}, 3.48 \mathrm{mmol}, 1.0 \mathrm{eq})$ and methyl 2-pyrone-5-carboxylate $(820 \mathrm{mg}$, $5.32 \mathrm{mmol}, 1.5 \mathrm{eq})$ in $\mathrm{CH}_{2} \mathrm{Cl}_{2}(5 \mathrm{ml})$ was pressurized to $14 \mathrm{kbar}$ for $7 \mathrm{~d}$. After evaporation of the volatiles the crude product was purified by column chromatography (PE-EtOAc= 20:1 $\rightarrow 5: 1 \rightarrow 2: 1$ ) to afford the cycloaddition products SI-10 (1.4 g, $2.98 \mathrm{mmol}, 86 \% \mathrm{dr}=4: 12: 2: 6)$ as yellow semi-solid materials.

SI-10a: $\mathbf{R}_{\mathbf{f}}=0.53\left(\right.$ PE-EtOAc 1:1); ${ }^{1} \mathrm{H}-\mathrm{NMR}\left(400 \mathrm{MHz} ; \mathrm{CDCl}_{3} ; \mathrm{CHCl}_{3}=7.26 \mathrm{ppm}\right) \delta_{\mathrm{H}}=7.37(1 \mathrm{H}, \mathrm{dd}, J=$ 6.5, $\left.2.3 \mathrm{~Hz}, \mathrm{H}_{13}\right), 5.74-5.79\left(1 \mathrm{H}, \mathrm{m}, \mathrm{H}_{11}\right), 4.70(1 \mathrm{H}, \mathrm{d}, J=6.9 \mathrm{~Hz}, \mathrm{OMOM}), 4.60(1 \mathrm{H}, \mathrm{d}, J=6.9 \mathrm{~Hz}$, OMOM), $4.47\left(1 \mathrm{H}, \mathrm{dd}, J=7.4,4.0 \mathrm{~Hz}, \mathrm{H}_{15}\right), 4.01\left(1 \mathrm{H}, \mathrm{dd}, J=6.3,4.0 \mathrm{~Hz}, \mathrm{H}_{14}\right), 3.78-3.86\left(4 \mathrm{H}, \mathrm{m}, \mathrm{H}_{6}\right.$, $\left.\mathrm{H}_{22}\right), 3.36(3 \mathrm{H}, \mathrm{s}, \mathrm{OMOM}), 3.31\left(1 \mathrm{H}, \mathrm{dd}, J=9.4,5.0 \mathrm{~Hz}, \mathrm{H}_{7}\right), 3.07\left(1 \mathrm{H}, \mathrm{dd}, J=10.5,8.9 \mathrm{~Hz}, \mathrm{H}_{9}\right), 2.40(1 \mathrm{H}$, $\left.\mathrm{ddd}, J=8.9,7.4,2.9 \mathrm{~Hz}, \mathrm{H}_{10}\right), 1.63-1.75\left(1 \mathrm{H}, \mathrm{m}, \mathrm{H}_{8}\right), 1.21\left(3 \mathrm{H}, \mathrm{d}, J=6.4 \mathrm{~Hz}, \mathrm{H}_{18}\right), 1.04(3 \mathrm{H}, \mathrm{d}, J=6.4 \mathrm{~Hz}$, $\left.\mathrm{H}_{19}\right), 0.94(9 \mathrm{H}, \mathrm{s}, \mathrm{TBS}), 0.26$ (3H, s, TBS), $0.10(3 \mathrm{H}, \mathrm{s}, \mathrm{TBS}) ;{ }^{13} \mathrm{C}-\mathrm{NMR}\left(101 \mathrm{MHz} ; \mathrm{CDCl}_{3} ; \mathrm{CDCl}_{3}=77.16\right.$ ppm) $\delta_{C}=170.1\left(\mathrm{~s}, \mathrm{C}_{23}\right), 163.2\left(\mathrm{~s}, \mathrm{C}_{20}\right), 142.1$ (d, $\mathrm{C}_{13}$ ), 133.9 (s, $\mathrm{C}_{12}$ ), 95.1 (t, OMOM), 82.7 (d, C 7 ), 74.6 $\left(d, C_{11}\right), 74.6\left(d, C_{6}\right), 72.7\left(s, C_{9}\right), 68.3\left(s, C_{15}\right), 55.8$ (q, OMOM), 52.4 (q, $C_{22}$ ), $50.4\left(s, C_{10}\right), 49.0\left(d, C_{14}\right)$, $37.9\left(\mathrm{~d}, \mathrm{C}_{8}\right), 26.4$ (q, TBS), 18.7 (s, TBS), 17.4 ( $q, \mathrm{C}_{18}$ ), 16.2 (q, $\mathrm{C}_{19}$ ), -3.5 (q, TBS), -3.7 (q, TBS).

SI-10b: $\mathbf{R}_{\mathbf{f}}=0.38\left(\right.$ PE-EtOAc 1:1); ${ }^{1} \mathrm{H}-\mathrm{NMR}\left(400 \mathrm{MHz} ; \mathrm{CDCl}_{3} ; \mathrm{CHCl}_{3}=7.26 \mathrm{ppm}\right) \delta_{\mathrm{H}}=7.19(1 \mathrm{H}, \mathrm{dd}, J=$ 6.6, $\left.2.2 \mathrm{~Hz}, \mathrm{H}_{13}\right), 5.68-5.79\left(1 \mathrm{H}, \mathrm{m}, \mathrm{H}_{11}\right), 4.70(1 \mathrm{H}, \mathrm{d}, J=6.9 \mathrm{~Hz}, \mathrm{OMOM}), 4.58(1 \mathrm{H}, \mathrm{d}, J=6.9 \mathrm{~Hz}$, OMOM), $4.12\left(1 \mathrm{H}, \mathrm{dd}, J=9.0,3.8 \mathrm{~Hz}, \mathrm{H}_{15}\right), 3.76-3.87\left(5 \mathrm{H}, \mathrm{m}, \mathrm{H}_{6}, \mathrm{H}_{14}, \mathrm{H}_{22}\right), 3.69(1 \mathrm{H}, \mathrm{dd}, J=10.6,9.4$ $\left.\mathrm{Hz}, \mathrm{H}_{7}\right), 3.45\left(1 \mathrm{H}, \mathrm{dd}, J=8.5,6.7 \mathrm{~Hz}, \mathrm{H}_{9}\right), 3.35(3 \mathrm{H}, \mathrm{s}, \mathrm{OMOM}), 1.73-1.78\left(1 \mathrm{H}, \mathrm{m}, \mathrm{H}_{10}\right), 1.64-1.73(1 \mathrm{H}$, $\left.m, H_{8}\right), 1.22\left(3 \mathrm{H}, \mathrm{d}, J=6.3 \mathrm{~Hz}, \mathrm{H}_{18}\right), 1.11\left(3 \mathrm{H}, \mathrm{d}, J=6.5 \mathrm{~Hz}, \mathrm{H}_{18}\right), 0.96(9 \mathrm{H}, \mathrm{s}, \mathrm{TBS}), 0.24(3 \mathrm{H}, \mathrm{s}, \mathrm{TBS})$, $0.12(3 \mathrm{H}, \mathrm{s}, \mathrm{TBS}) ;{ }^{13} \mathrm{C}-\mathrm{NMR}\left(101 \mathrm{MHz} ; \mathrm{CDCl}_{3} ; \mathrm{CDCl}_{3}=77.16 \mathrm{ppm}\right) \delta_{\mathrm{C}}=170.1\left(\mathrm{~s}, \mathrm{C}_{23}\right), 162.3\left(\mathrm{~s}, \mathrm{C}_{22}\right), 138.8$

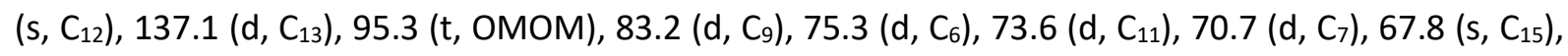
55.8 (q, OMOM), 52.4 (q, $C_{22}$ ), 48.9 (s, $C_{10}$ ), 46.7 (s, $C_{14}$ ), 39.0 (d, $C_{8}$ ), 26.1 (s, TBS), 18.4 (q, $C_{18}$ ), 18.4 (s, TBS), 17.6 ( $\left(, C_{19}\right),-3.2(q, T B S),-4.2(q, T B S)$. 
SI-10c: $\mathbf{R}_{\mathbf{f}}=0.25$ (PE-EtOAc 1:1); ${ }^{1} \mathbf{H}-\mathrm{NMR}\left(400 \mathrm{MHz} ; \mathrm{CDCl}_{3} ; \mathrm{CHCl}_{3}=7.26 \mathrm{ppm}\right) \delta_{\mathrm{H}}=7.38(1 \mathrm{H}, \mathrm{dd}, \mathrm{J}=$ 6.3, $\left.1.9 \mathrm{~Hz}, \mathrm{H}_{13}\right), 5.88-5.93\left(1 \mathrm{H}, \mathrm{m}, \mathrm{H}_{11}\right), 4.66(1 \mathrm{H}, \mathrm{d}, J=7.0 \mathrm{~Hz}, \mathrm{OMOM}), 4.62(1 \mathrm{H}, \mathrm{d}, J=7.0 \mathrm{~Hz}$, OMOM), 4.50-4.58 $\left(1 \mathrm{H}, \mathrm{m}, \mathrm{H}_{15}\right), 4.26\left(1 \mathrm{H}, \mathrm{dd}, J=6.3,4.7 \mathrm{~Hz}, \mathrm{H}_{14}\right), 3.81\left(3 \mathrm{H}, \mathrm{s}, \mathrm{H}_{22}\right), 3.78(1 \mathrm{H}, \mathrm{dq}, J=$ 6.7, $\left.2.0 \mathrm{~Hz}, \mathrm{H}_{6}\right), 3.69\left(1 \mathrm{H}, \mathrm{dd}, J=9.9,2.0 \mathrm{~Hz}, \mathrm{H}_{7}\right), 3.35(3 \mathrm{H}, \mathrm{s}, \mathrm{OMOM}), 3.18\left(1 \mathrm{H}, \mathrm{dd}, J=9.9,2.0 \mathrm{~Hz}, \mathrm{H}_{9}\right)$, 2.51-2.58 (1H, m, $\left.\mathrm{H}_{10}\right), 1.20-1.35\left(1 \mathrm{H}, \mathrm{m}, \mathrm{H}_{8}\right), 0.96-1.04\left(12 \mathrm{H}, \mathrm{m}, \mathrm{H}_{18}, \mathrm{TBS}\right), 0.77\left(1 \mathrm{H}, \mathrm{d}, \mathrm{J}=6.5 \mathrm{~Hz}, \mathrm{H}_{19}\right)$, $0.17(3 \mathrm{H}, \mathrm{s}, \mathrm{TBS}), 0.11(3 \mathrm{H}, \mathrm{s}, \mathrm{TBS})$.

SI-10d: $\mathbf{R}_{\mathrm{f}}=0.07$ (PE-EtOAc 1:1); ${ }^{1} \mathbf{H}-\mathrm{NMR}\left(400 \mathrm{MHz} ; \mathrm{CDCl}_{3} ; \mathrm{CHCl}_{3}=7.26 \mathrm{ppm}\right) \delta_{\mathrm{H}}=7.16(1 \mathrm{H}, \mathrm{dd}, \mathrm{J}=$ 6.5, $\left.2.4 \mathrm{~Hz}, \mathrm{H}_{13}\right), 6.05\left(1 \mathrm{H}, \mathrm{b}, \mathrm{H}_{11}\right), 4.70(1 \mathrm{H}, \mathrm{d}, J=7.0 \mathrm{~Hz}, \mathrm{OMOM}), 4.65(1 \mathrm{H}, \mathrm{d}, J=7.0 \mathrm{~Hz}, \mathrm{OMOM})$, $4.21\left(1 \mathrm{H}, \mathrm{dd}, J=7.4,4.4 \mathrm{~Hz}, \mathrm{H}_{15}\right), 4.12\left(1 \mathrm{H}, \mathrm{dd}, J=6.5,4.4 \mathrm{~Hz}, \mathrm{H}_{14}\right), 3.85\left(1 \mathrm{H}, \mathrm{qd}, J=6.5,2.3 \mathrm{~Hz}, \mathrm{H}_{6}\right)$, $3.79\left(3 \mathrm{H}, \mathrm{s}, \mathrm{H}_{22}\right), 3.74(1 \mathrm{H}, \mathrm{dd}, J=10.4,7.4 \mathrm{~Hz}, \mathrm{H}-9), 3.37(3 \mathrm{H}, \mathrm{s}, \mathrm{OMOM}), 3.29(1 \mathrm{H}, \mathrm{dd}, J=9.9,2.3 \mathrm{~Hz}$, $\left.\mathrm{H}_{7}\right), 1.82-1.91\left(1 \mathrm{H}, \mathrm{m}, \mathrm{H}_{10}\right), 1.60-1.73\left(1 \mathrm{H}, \mathrm{m}, \mathrm{H}_{8}\right), 1.13\left(3 \mathrm{H}, \mathrm{d}, J=6.5 \mathrm{~Hz}, \mathrm{H}_{18}\right), 1.00(9 \mathrm{H}, \mathrm{s}, \mathrm{TBS}), 0.87$ $\left(3 \mathrm{H}, \mathrm{d}, \mathrm{J}=6.6 \mathrm{~Hz}, \mathrm{H}_{19}\right), 0.11(3 \mathrm{H}, \mathrm{s}, \mathrm{TBS}), 0.06(3 \mathrm{H}, \mathrm{s}, \mathrm{TBS})$. 
Methyl (3S,4R)-4-((tert-butyldimethylsilyl)oxy)-2-((S)-1-(methoxymethoxy)ethyl)-3-methylchromane-6-carboxylate (38)

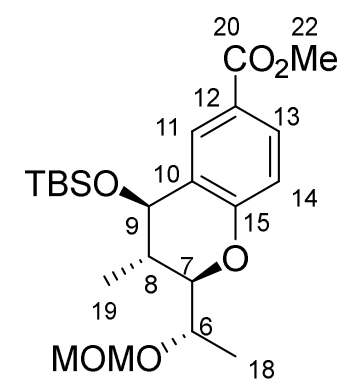

38

A solution of methyl ester $\mathbf{S I}-10(2.0 \mathrm{~g}, 4.25 \mathrm{mmol}, 1.0 \mathrm{eq})$ in toluene $(10 \mathrm{ml})$ was heated to $160{ }^{\circ} \mathrm{C}$ in a sealed tube over $24 \mathrm{~h}$. Evaporation of the solvent and purification by column chromatoraphy (PEEtOAc $=10: 1)$ afforded the decarboxylated product $(1.45 \mathrm{~g}, 3.40 \mathrm{mmol}, 83 \%)$ as a yellow oil. Thismaterial (1.45 g, $3.40 \mathrm{mmol}, 1.0 \mathrm{eq})$ was dissolved in $\mathrm{CH}_{2} \mathrm{Cl}_{2}(40 \mathrm{ml})$ and cooled to $0{ }^{\circ} \mathrm{C}$ and a solution of DDQ (930 mg, $4.10 \mathrm{mmol}, 1.2 \mathrm{eq})$ in $\mathrm{CH}_{2} \mathrm{Cl}_{2}(20 \mathrm{ml})$ was added dropwise. After stirring for $12 \mathrm{~h}$ at $\mathrm{rt}$ the reaction was terminated by addition of a sat. aq. $\mathrm{Na}_{2} \mathrm{~S}_{2} \mathrm{O}_{3}$ solution and a sat. aq. $\mathrm{NaHCO}_{3}$ solution. Layers were separated and the aqueous layer was extracted with $\mathrm{CH}_{2} \mathrm{Cl}_{2}$. The combined organic phases were washed with $1 \mathrm{M}$ aq. $\mathrm{NaOH}$, water and brine, dried over $\mathrm{MgSO}_{4}$, filtered and evaporated under reduced pressure. The crude product was purified by column chromatography (PE-EtOAc= 10:1) to afford chromane $38(1.3 \mathrm{~g}, 3.06 \mathrm{mmol}, 90 \%)$ as a colorless oil.

$\mathbf{R}_{\mathbf{f}}=0.36\left(\right.$ PE-EtOAc 5:1); $[\alpha]^{26}{ }_{\mathrm{D}}=-32.6^{\circ}\left(c=1.00 ; \mathrm{CH}_{2} \mathrm{Cl}_{2}\right) ;{ }^{1} \mathrm{H}-\mathrm{NMR}\left(400 \mathrm{MHz} ; \mathrm{CDCl}_{3} ; \mathrm{CHCl}_{3}=7.26\right.$ ppm) $\delta_{H}=8.10\left(1 \mathrm{H}, \mathrm{dd}, J=2.1,0.9 \mathrm{~Hz}, \mathrm{H}_{11}\right), 7.84\left(1 \mathrm{H}, \mathrm{dd}, J=8.6,2.1 \mathrm{~Hz}, \mathrm{H}_{13}\right), 6.91(1 \mathrm{H}, \mathrm{d}, J=8.6 \mathrm{~Hz}$, $\left.\mathrm{H}_{14}\right), 4.65-4.82(2 \mathrm{H}, \mathrm{m}, \mathrm{OMOM}), 4.48\left(1 \mathrm{H}, \mathrm{d}, J=8.9 \mathrm{~Hz}, \mathrm{H}_{9}\right), 4.14\left(1 \mathrm{H}, \mathrm{dd}, J=9.9,2.7 \mathrm{~Hz}, \mathrm{H}_{7}\right), 4.06(1 \mathrm{H}$, $\left.\mathrm{qd}, J=6.4,2.7 \mathrm{~Hz}, \mathrm{H}_{6}\right), 3.86\left(3 \mathrm{H}, \mathrm{s}, \mathrm{H}_{22}\right), 3.40(3 \mathrm{H}, \mathrm{s}, \mathrm{OMOM}), 1.80-1.98\left(1 \mathrm{H}, \mathrm{m}, \mathrm{H}_{8}\right), 1.24(3 \mathrm{H}, \mathrm{d}, J=6.4$ $\left.\mathrm{Hz}, \mathrm{H}_{18}\right), 1.09\left(3 \mathrm{H}, \mathrm{d}, J=6.7 \mathrm{~Hz}, \mathrm{H}_{19}\right), 1.00$ (9H, s, TBS), 0.25 (3H, s, TBS), 0.16 (3H, s, TBS); ${ }^{13} \mathrm{C}-\mathrm{NMR}$ $\left(101 \mathrm{MHz} ; \mathrm{CDCl}_{3} ; \mathrm{CDCl}_{3}=77.16 \mathrm{ppm}\right) \delta_{\mathrm{C}}=167.1\left(\mathrm{~s}, \mathrm{C}_{20}\right), 158.1\left(\mathrm{~s}, \mathrm{C}_{15}\right), 130.5\left(\mathrm{~d}, \mathrm{C}_{11}\right), 129.6\left(\mathrm{~d}, \mathrm{C}_{13}\right)$, $126.3\left(\mathrm{~s}, \mathrm{C}_{10}\right), 122.3$ (s, $\mathrm{C}_{12}$ ), 116.5 (d, $\mathrm{C}_{14}$ ), 95.5 (t, OMOM), 82.4 (d, $\mathrm{C}_{7}$ ), 73.1 (d, $\mathrm{C}_{9}$ ), 71.3 (d, $\mathrm{C}_{6}$ ), 55.6 (q, OMOM), 51.9 (q, $C_{22}$ ), 37.0 (d, $C_{8}$ ), 26.1 (q, TBS), 18.4 (s, TBS), 14.5 (q, $\left.C_{18}\right), 13.8$ (q, $C_{19}$ ), -3.5 (q, TBS), -3.7 (q, TBS); HRMS (ESI): $m / z$ calc. for $\mathrm{C}_{22} \mathrm{H}_{36} \mathrm{O}_{6} \mathrm{Si}[\mathrm{M}+\mathrm{Na}]^{+}: 447.2179$, found 447.2176. 
<smiles>COC(C)C1Oc2ccc(C=O)cc2[C@@H](OC(C)C)[C@@H]1C</smiles>

39

To a solution of methyl ester $38(40 \mathrm{mg}, 0.094 \mathrm{mmol}, 1.0 \mathrm{eq})$ in THF (2 ml) was added DIBAI-H solution $1 \mathrm{M}$ in THF $(0.23 \mathrm{ml}, 0.23 \mathrm{mmol}, 2.4 \mathrm{eq})$ dropwise at $-78{ }^{\circ} \mathrm{C}$.After stirring for $30 \mathrm{~min}$ at $0{ }^{\circ} \mathrm{C}$ the reaction was termiated by addition of methanol. The mixture was diluted with EtOAc and a sat. aq. Rochelle salt solution was added and stirring was continued for $2 \mathrm{~h}$ at $\mathrm{rt}$. Layers were separated and the aqueous layer was extracted with EtOAc. The combined organic phases were dried over $\mathrm{MgSO}_{4}$ and evaporated under reduced pressure. The crude product was dissolved in $\mathrm{CH}_{2} \mathrm{Cl}_{2}(5 \mathrm{ml})$ and $\mathrm{MnO}_{2}(154 \mathrm{mg}, 1.8 \mathrm{mmol}, 19 \mathrm{eq})$ was added. After stirring for $12 \mathrm{~h}$ at $\mathrm{rt}$, the mixture was filtered over Celite $^{\circledR}$ and the solvent was evaporated. Purification by column chromatography (PE-EtOAc= 10:1) afforded benzaldehyde 39 (35 mg, 0.089 mmol, $95 \%$ ) as a colorless oil.

$\mathbf{R}_{\mathbf{f}}=0.14\left(\right.$ PE-EtOAc 5:1); $[\alpha]^{26}{ }_{\mathrm{D}}=-30.8^{\circ}\left(c=1.00 ; \mathrm{CH}_{2} \mathrm{Cl}_{2}\right) ;{ }^{1} \mathrm{H}-\mathrm{NMR}\left(400 \mathrm{MHz} ; \mathrm{CDCl}_{3} ; \mathrm{CHCl}_{3}=7.26\right.$ ppm) $\delta_{\mathrm{H}}=9.84\left(1 \mathrm{H}, \mathrm{s}, \mathrm{H}_{20}\right), 7.88\left(1 \mathrm{H}, \mathrm{d}, J=2.0 \mathrm{~Hz}, \mathrm{H}_{11}\right), 7.71\left(1 \mathrm{H}, \mathrm{dd}, J=8.4,2.0 \mathrm{~Hz}, \mathrm{H}_{13}\right), 7.00(1 \mathrm{H}, \mathrm{d}, J$ $\left.=8.4 \mathrm{~Hz}, \mathrm{H}_{14}\right), 4.74-4.77(2 \mathrm{H}, \mathrm{m}, \mathrm{OMOM}), 4.51\left(1 \mathrm{H}, \mathrm{d}, J=9.0 \mathrm{~Hz}, \mathrm{H}_{9}\right), 4.17\left(1 \mathrm{H}, \mathrm{dd}, J=9.8,2.9 \mathrm{~Hz}, \mathrm{H}_{7}\right)$, $4.06\left(1 \mathrm{H}, \mathrm{dq}, J=6.4,2.9 \mathrm{~Hz}, \mathrm{H}_{6}\right), 3.41(3 \mathrm{H}, \mathrm{s}, \mathrm{OMOM}), 1.87-1.96\left(1 \mathrm{H}, \mathrm{m}, \mathrm{H}_{8}\right), 1.25(3 \mathrm{H}, \mathrm{d}, J=6.4 \mathrm{~Hz}$, $\left.\mathrm{H}_{18}\right), 1.10\left(3 \mathrm{H}, \mathrm{d}, J=6.7 \mathrm{~Hz}, \mathrm{H}_{19}\right), 1.00$ (9H, s, TBS), 0.25 (3H, s, TBS), 0.17 (3H, s, TBS); ${ }^{13} \mathrm{C}-\mathrm{NMR}(101$ $\left.\mathrm{MHz} ; \mathrm{CDCl}_{3} ; \mathrm{CDCl}_{3}=77.16 \mathrm{ppm}\right) \delta_{\mathrm{c}}=190.8\left(\mathrm{~d}, \mathrm{C}_{20}\right), 159.5\left(\mathrm{~s}, \mathrm{C}_{15}\right), 130.4\left(\mathrm{~s}, \mathrm{C}_{12}\right), 130.2\left(\mathrm{~d}, \mathrm{C}_{13}\right), 129.8$ (d, $C_{11}$ ), 127.1 (s, $\left.C_{10}\right), 117.3$ (d, $\left.C_{14}\right), 95.5$ (t, OMOM), 82.7 (d, $\left.C_{6}\right), 73.0$ (d, $\left.C_{7}\right), 71.1$ (d, $\left.C_{9}\right), 55.6$ (q) OMOM), 37.0 ( $d, C_{8}$ ), 26.1 (3C, q, TBS), 18.5 (s, TBS), 14.5 (q, $C_{18}$ ), 13.9 (q, $C_{19}$ ), -3.5 (q, TBS), -3.6 (q,TBS); HRMS (ESI): $m / z$ calc. for $\mathrm{C}_{21} \mathrm{H}_{34} \mathrm{O}_{5} \mathrm{Si}[\mathrm{M}+\mathrm{Na}]^{+}:$417.2073, found 417.2076. 


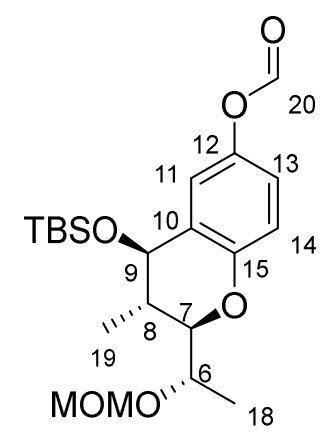

40

A solution of aldehyde 39 ( $28 \mathrm{mg}, 0.07 \mathrm{mmol}, 1.0 \mathrm{eq})$ in $\mathrm{CH}_{2} \mathrm{Cl}_{2}(3 \mathrm{ml})$ was heated to $40{ }^{\circ} \mathrm{C}$. Then, 3chloroperbenzoic acid (77\%, $45 \mathrm{mg}, 0.21 \mathrm{mmol}, 3.0 \mathrm{eq})$ was added portionswise over $4 \mathrm{~h}$. After stirring for $12 \mathrm{~h}$ at $40{ }^{\circ} \mathrm{C}$ the reaction was terminated by addition of a sat. aq. $\mathrm{Na}_{2} \mathrm{~S}_{2} \mathrm{O}_{3}$ solution and a sat. aq. $\mathrm{NaHCO}_{3}$ solution. Layers were separated and the aqueous layer was extracted with $\mathrm{CH}_{2} \mathrm{Cl}_{2}$. The combined organic phases were washed with water and with brine, dried over $\mathrm{MgSO}_{4}$, filtered and the solvent was evaporated. Purification by column chromatography (PE-EtOAc=10:1) afforded formyl ester $\mathbf{4 0}$ (28.5 mg, $0.07 \mathrm{mmol}$, quant.) as a colorless oil.

$\mathbf{R}_{\mathbf{f}}=0.32\left(\right.$ PE-EtOAC 5:1); $[\alpha]^{26}{ }_{\mathrm{D}}=-22.3^{\circ}\left(c=1.00 ; \mathrm{CH}_{2} \mathrm{Cl}_{2}\right) ;{ }^{1} \mathrm{H}-\mathrm{NMR}\left(400 \mathrm{MHz} ; \mathrm{CDCl}_{3} ; \mathrm{CHCl}_{3}=7.26\right.$ ppm) $\delta_{H}=8.27\left(1 \mathrm{H}, \mathrm{s}, \mathrm{H}_{20}\right), 7.08-7.09\left(1 \mathrm{H}, \mathrm{m}, \mathrm{H}_{11}\right), 6.89-6.92\left(2 \mathrm{H}, \mathrm{m}, \mathrm{H}-13, \mathrm{H}_{14}\right), 4.74(2 \mathrm{H}, \mathrm{s}, \mathrm{OMOM})$, $4.47\left(1 \mathrm{H}, \mathrm{d}, J=8.9 \mathrm{~Hz}, \mathrm{H}_{9}\right), 4.06\left(1 \mathrm{H}, \mathrm{dd}, J=9.5,3.0 \mathrm{~Hz}, \mathrm{H}_{7}\right), 4.02\left(1 \mathrm{H}, \mathrm{dq}, J=6.4,3.0 \mathrm{~Hz}, \mathrm{H}_{6}\right), 3.40(3 \mathrm{H}$, s, OMOM), 1.85-1.95 (1H, m, H8), $1.24\left(3 \mathrm{H}, \mathrm{d}, J=6.4 \mathrm{~Hz}, \mathrm{H}_{18}\right), 1.08\left(3 \mathrm{H}, \mathrm{d}, J=6.7 \mathrm{~Hz}, \mathrm{H}_{19}\right), 0.97(9 \mathrm{H}, \mathrm{s}$, TBS), 0.19 (3H, s, TBS), $0.14(3 \mathrm{H}, \mathrm{s}, \mathrm{TBS}) ;{ }^{13} \mathrm{C}-\mathrm{NMR}\left(101 \mathrm{MHz} ; \mathrm{CDCl}_{3} ; \mathrm{CDCl}_{3}=77.16 \mathrm{ppm}\right) \delta_{\mathrm{C}}=159.8$ (d,

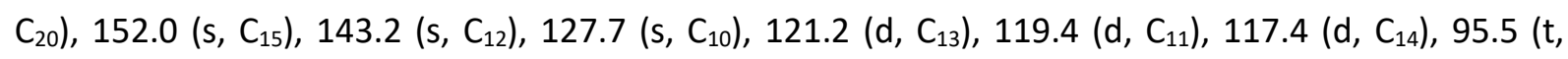
OMOM), 82.0 (d, $C_{6}$ ), 73.3 (d, $C_{7}$ ), 71.3 (d, $C_{9}$ ), 55.6 (q, OMOM), 37.2 (d, $C_{8}$ ), 26.1 (q, TBS), 18.4 (s, TBS), 14.7 ( $q, \mathrm{C}_{18}$ ), 14.0 (q, $\mathrm{C}_{19}$ ), -3.5 ( $q, \mathrm{TBS}$ ), -3.7 (q, TBS) HRMS (ESI): $\mathrm{m} / \mathrm{z}$ calc. for $\mathrm{C}_{21} \mathrm{H}_{34} \mathrm{O}_{6} \mathrm{Si}$ $[\mathrm{M}+\mathrm{Na}]^{+}:$433.2022, found 433.2022. 
1,1'-((2R,3S,4R)-4-((Tert-butyldimethylsilyl)oxy)-2-((S)-1-(methoxymethoxy)ethyl)-3-methyl-3,4dihydro-6,8a-ethanochromene-6,8(2H)-diyl)bis(ethan-1-one) (32)

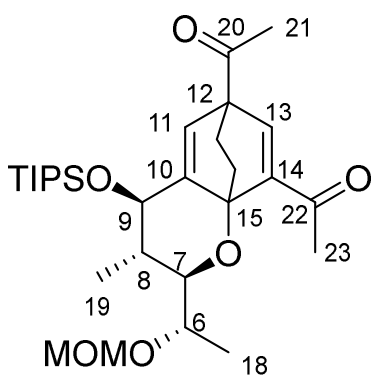

32

$\mathbf{R}_{\mathbf{f}}=0.10\left(\right.$ PE-EtOAC 2:1); ${ }^{1} \mathrm{H}-\mathrm{NMR}\left(400 \mathrm{MHz} ; \mathrm{CDCl}_{3} ; \mathrm{CHCl}_{3}=7.26 \mathrm{ppm}\right) \delta_{\mathrm{H}}=7.37-7.35(1 \mathrm{H}, \mathrm{m}, \mathrm{H} 13)$, $7.05\left(1 \mathrm{H}, \mathrm{d}, J=1.0 \mathrm{~Hz}, \mathrm{H}_{11}\right), 4.57(2 \mathrm{H}, \mathrm{s}, \mathrm{OMOM}), 4.25\left(1 \mathrm{H}, \mathrm{dd}, J=9.9,5.2 \mathrm{~Hz}, \mathrm{H}_{9}\right), 3.87(1 \mathrm{H}, \mathrm{dd}, J=$ 10.1, 3.6 Hz, $\left.\mathrm{H}_{7}\right), 3.82\left(1 \mathrm{H}, \mathrm{dq}, J=6.5,3.6 \mathrm{~Hz}, \mathrm{H}_{6}\right), 3.33\left(1 \mathrm{H}, \mathrm{dd}, J=8.0,6.9 \mathrm{~Hz}, \mathrm{H}_{23}\right), 3.28(3 \mathrm{H}, \mathrm{s}$, OMOM), $2.66\left(1 \mathrm{H}, \mathrm{ddd}, J=10.8,8.0,1.0 \mathrm{~Hz}, \mathrm{H}_{22}\right), 2.38-2.32\left(4 \mathrm{H}, \mathrm{m}, \mathrm{H}_{23}, \mathrm{H}_{25}\right), 2.27\left(3 \mathrm{H}, \mathrm{s}, \mathrm{H}_{21}\right), 1.57-$ $1.47\left(1 \mathrm{H}, \mathrm{m}, \mathrm{H}_{8}\right), 1.47-1.39\left(1 \mathrm{H}, \mathrm{m}, \mathrm{H}_{22}\right), 1.08\left(3 \mathrm{H}, \mathrm{d}, J=6.5 \mathrm{~Hz}, \mathrm{H}_{18}\right), 1.07-1.02\left(24 \mathrm{H}, \mathrm{TIPS}, \mathrm{H}_{19}\right) ;{ }^{13} \mathrm{C}-$ NMR (101 MHz; CDCl $; \mathrm{CDCl}_{3}=77.16$ ppm) $\delta_{\mathrm{c}}=197.1$ (s, $\left.\mathrm{C}_{20}\right), 194.4$ (s, $\left.\mathrm{C}_{24}\right), 151.2$ (s, $\left.\mathrm{C}_{10}\right), 149.5$ (d, $\mathrm{C}_{11}$ ), 143.9 /d, $\mathrm{C}_{13}$ ), 142.3 (s, $\mathrm{C}_{14}$ ), 94.8 (t, OMOM), 82.6 (s, $\mathrm{C}_{15}$ ), 79.6 (d, $\mathrm{C}_{7}$ ), 72.3 (d, $\mathrm{C}_{9}$ ), 72.2 (d, $\mathrm{C}_{6}$ ), 55.3 (q, OMOM), 47.1 (t, $C_{23}$ ), 47.0 (s, $C_{12}$ ), 38.0 (d, $C_{8}$ ), 26.0 (q, $C_{21}$ ), 25.5 (q, $C_{25}$ ), 24.1 (t, $C_{22}$ ), 18.3 (q, $\mathrm{C}_{18}$ ), 18.2 (q, $\mathrm{C}_{19}$ ), 13.2 (d, TIPS), 13.0 (q, TIPS), 12.9 (q, TIPS); HRMS (ESI): $\mathrm{m} / \mathrm{z}$ calc. for $\mathrm{C}_{29} \mathrm{H}_{48} \mathrm{O}_{6} \mathrm{Si}$ $[\mathrm{M}+\mathrm{Na}]^{+}: 543.3118$, found 543.3119 . 
1,1'-((2R,3S,4R)-4-((Tert-butyldimethylsilyl)oxy)-2-((S)-1-(methoxymethoxy)ethyl)-3methylchromane-6,8-diyl)bis(ethan-1-one) (33)

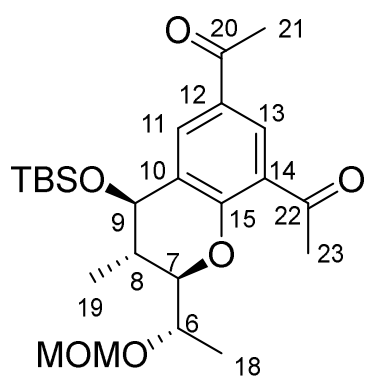

33

$\mathbf{R}_{\mathbf{f}}=0.20\left(\right.$ PE-EtOAC 5:1); $[\alpha]^{23}{ }_{\mathrm{D}}=-11.7^{\circ}\left(c=0.80 ; \mathrm{CH}_{2} \mathrm{Cl}_{2}\right) ;{ }^{1} \mathrm{H}-\mathrm{NMR}\left(400 \mathrm{MHz} ; \mathrm{CDCl}_{3} ; \mathrm{CHCl}_{3}=7.26\right.$ ppm) $\delta_{H}=8.27\left(1 \mathrm{H}, \mathrm{d}, J=2.3 \mathrm{~Hz}, \mathrm{H}_{11}\right), 8.18\left(1 \mathrm{H}, \mathrm{d}, J=\mathrm{H}_{13}\right), 4.77-4.69(2 \mathrm{H}, \mathrm{m}, \mathrm{OMOM}), 4.52(1 \mathrm{H}, \mathrm{d}, J=$ $\left.9.3 \mathrm{~Hz}, \mathrm{H}_{9}\right), 4.25\left(1 \mathrm{H}, \mathrm{dd}, J=10.0,2.7 \mathrm{~Hz}, \mathrm{H}_{7}\right), 4.08\left(1 \mathrm{H}, \mathrm{qd}, J=6.2,2.7 \mathrm{~Hz}, \mathrm{H}_{6}\right), 3.39(3 \mathrm{H}, \mathrm{s}, \mathrm{OMOM})$, $2.70\left(3 \mathrm{H}, \mathrm{s}, \mathrm{H}_{21}\right), 2.56\left(3 \mathrm{H}, \mathrm{s}, \mathrm{H}_{23}\right), 2.01-1.88\left(1 \mathrm{H}, \mathrm{m}, \mathrm{H}_{8}\right), 1.26\left(3 \mathrm{H}, \mathrm{d}, J=6.5 \mathrm{~Hz}, \mathrm{H}_{18}\right), 1.12(3 \mathrm{H}, \mathrm{d}, J=$ 6.7 Hz, $\left.\mathrm{H}_{19}\right), 1.00(9 \mathrm{H}, \mathrm{s}, \mathrm{TBS}), 0.26(3 \mathrm{H}, \mathrm{s}, \mathrm{TBS}), 0.17(3 \mathrm{H}, \mathrm{s}, \mathrm{TBS}) ;{ }^{13} \mathrm{C}-\mathrm{NMR}\left(101 \mathrm{MHz} ; \mathrm{CDCl}_{3} ; \mathrm{CDCl}_{3}=\right.$ $77.16 \mathrm{ppm}) \delta_{c}=198.9\left(\mathrm{~s}, \mathrm{C}_{20}\right), 196.4\left(\mathrm{~s}, \mathrm{C}_{22}\right), 157.9\left(\mathrm{~s}, \mathrm{C}_{15}\right), 131.5\left(\mathrm{~d}, \mathrm{C}_{13}\right), 130.9\left(\mathrm{~d}, \mathrm{C}_{11}\right), 129.6\left(\mathrm{~s}, \mathrm{C}_{14}\right)$, $128.2\left(\mathrm{~s}, \mathrm{C}_{12}\right.$ ), 126.9 (s, $\mathrm{C}_{10}$ ), 95.5 (t, OMOM), 83.3 (d, $\mathrm{C}_{7}$ ), 72.9 (d, $\left.\mathrm{C}_{6}\right), 71.4$ (d, $\mathrm{C}_{9}$ ), 55.6 (q, OMOM), $36.7\left(d, C_{8}\right), 32.5$ (q, $C_{21}$ ), 26.5 (q, $\left.C_{23}\right), 26.1$ (q, TBS), 18.4 (s, TBS), 14.3 (q, $\left.C_{18}\right), 14.1$ (q, $C_{19}$ ), -3.5 (q, TBS), -3.6 (q, TBS); HRMS (ESI): $m / z$ calc. for $\mathrm{C}_{24} \mathrm{H}_{38} \mathrm{O}_{6} \mathrm{Si}[\mathrm{M}+\mathrm{Na}]^{+}: 473.2335$, found 473.2339. 


\section{References (supporting information)}

(S1) Geist, E., Studien zur Totalsynthese der Cebulactame A1 und A2, PhD thesis, Leibniz Universität Hannover.

(S2) Urpí, F., Vilarrasa, J., Rovira, R., Ferreró, M., Galobardes, M., Martín, Montes, T., Romea, P. Synthesis, 2000, 11, 1608-1641.

(S3) Pale, P., Kern, N., Hoffmann, M., Blanc, A., Weibel, J.-M. Org. Lett. 2013, 15, 836-839.

(S4) Perrio, S., Gelat, F. G., Jayashankaran, J., Lohier, J.-F., Gaumont, A.-C. Org. Lett., 2011, 13, 3170-3173.

(S5) Ono, N., Kamikura, A., Kaji, A. J. Org. Chem., 1986, 15, 2139-2142.

(S6) Hirsch, J. A., Schwartzkopf, G. J. Org. Chem., 1973, 3534-3536. 
3 Attachment: Copies of $1 \mathrm{H}$ - and 13C-NMR spectra 

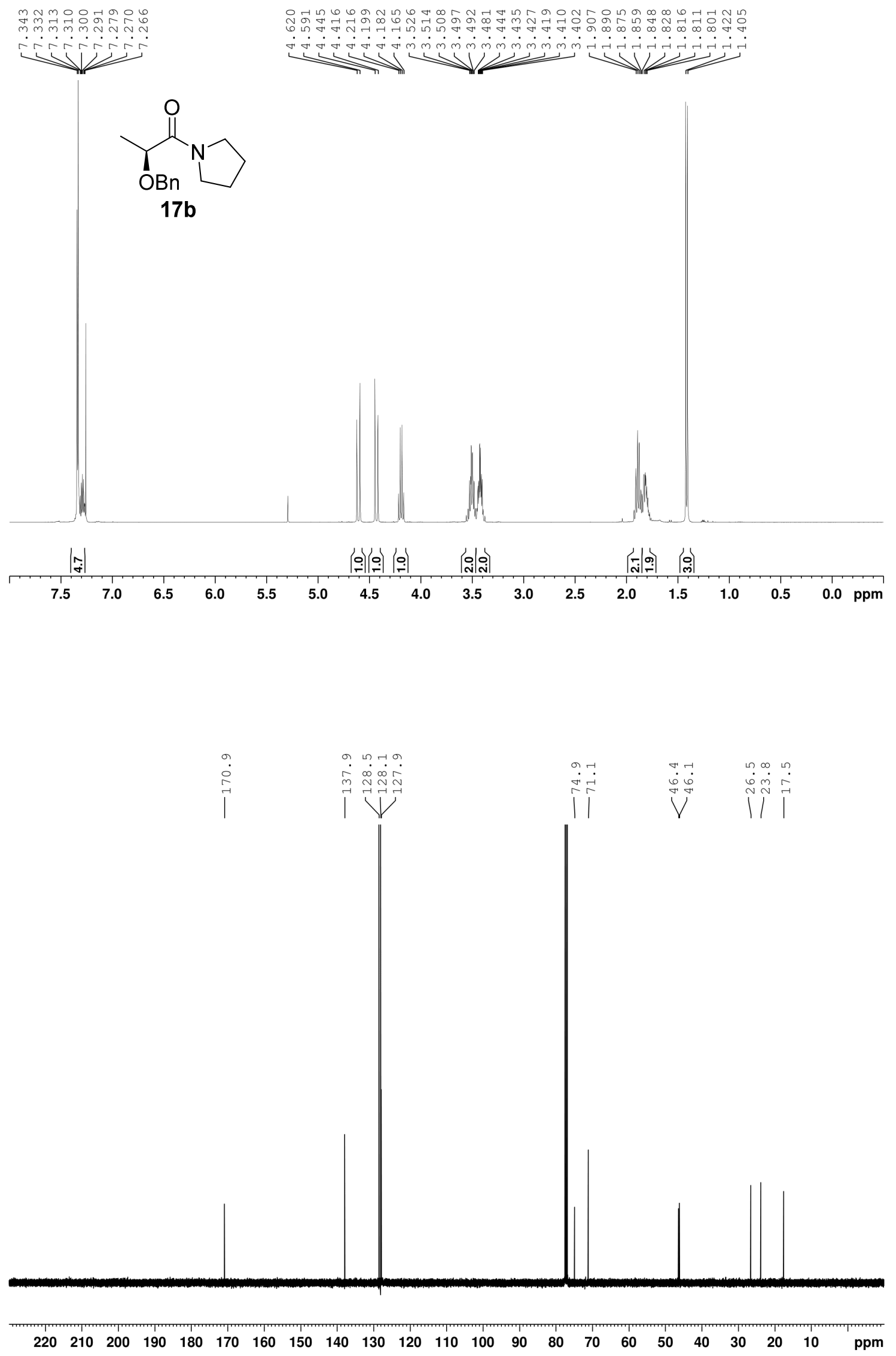

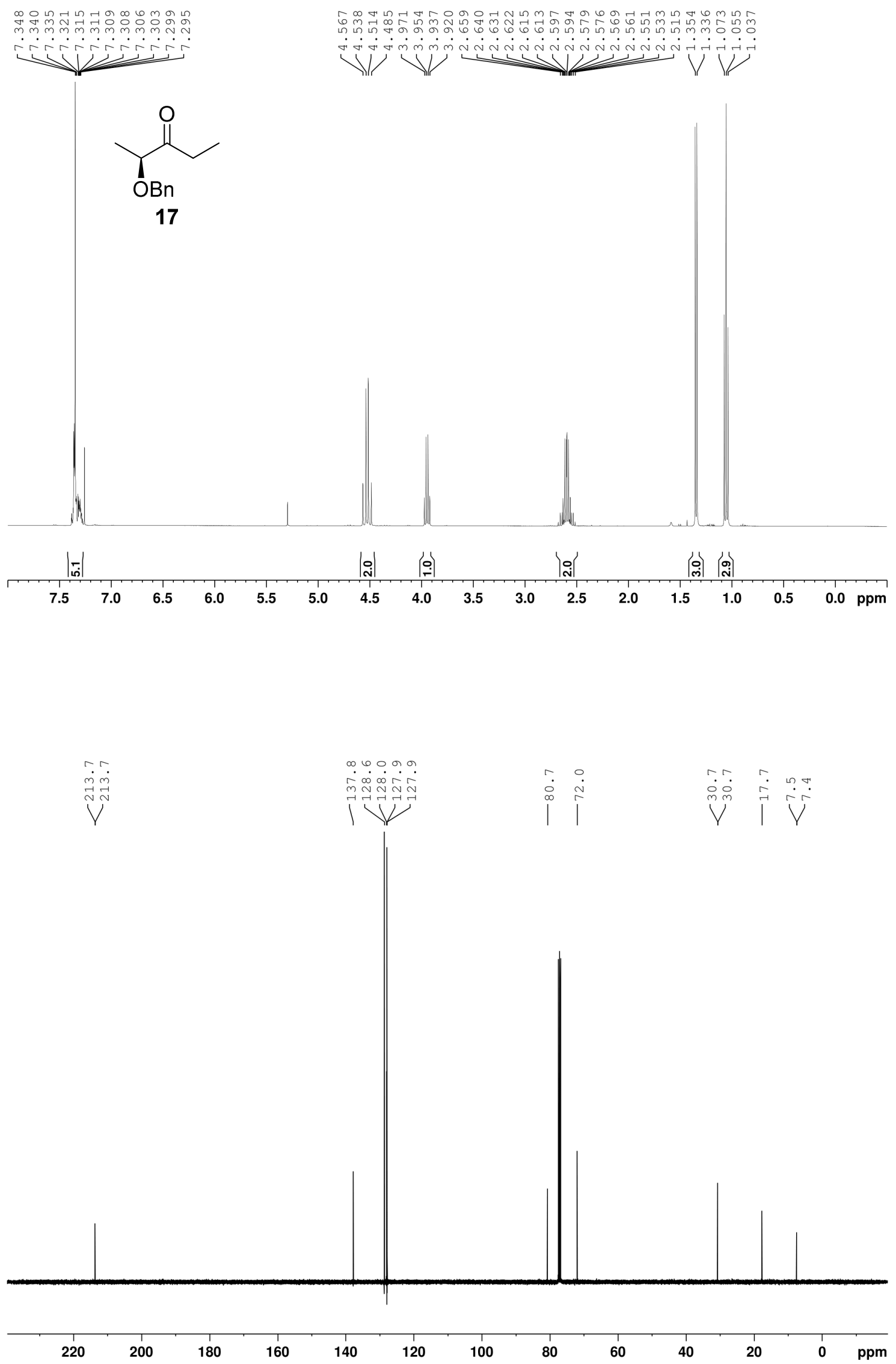


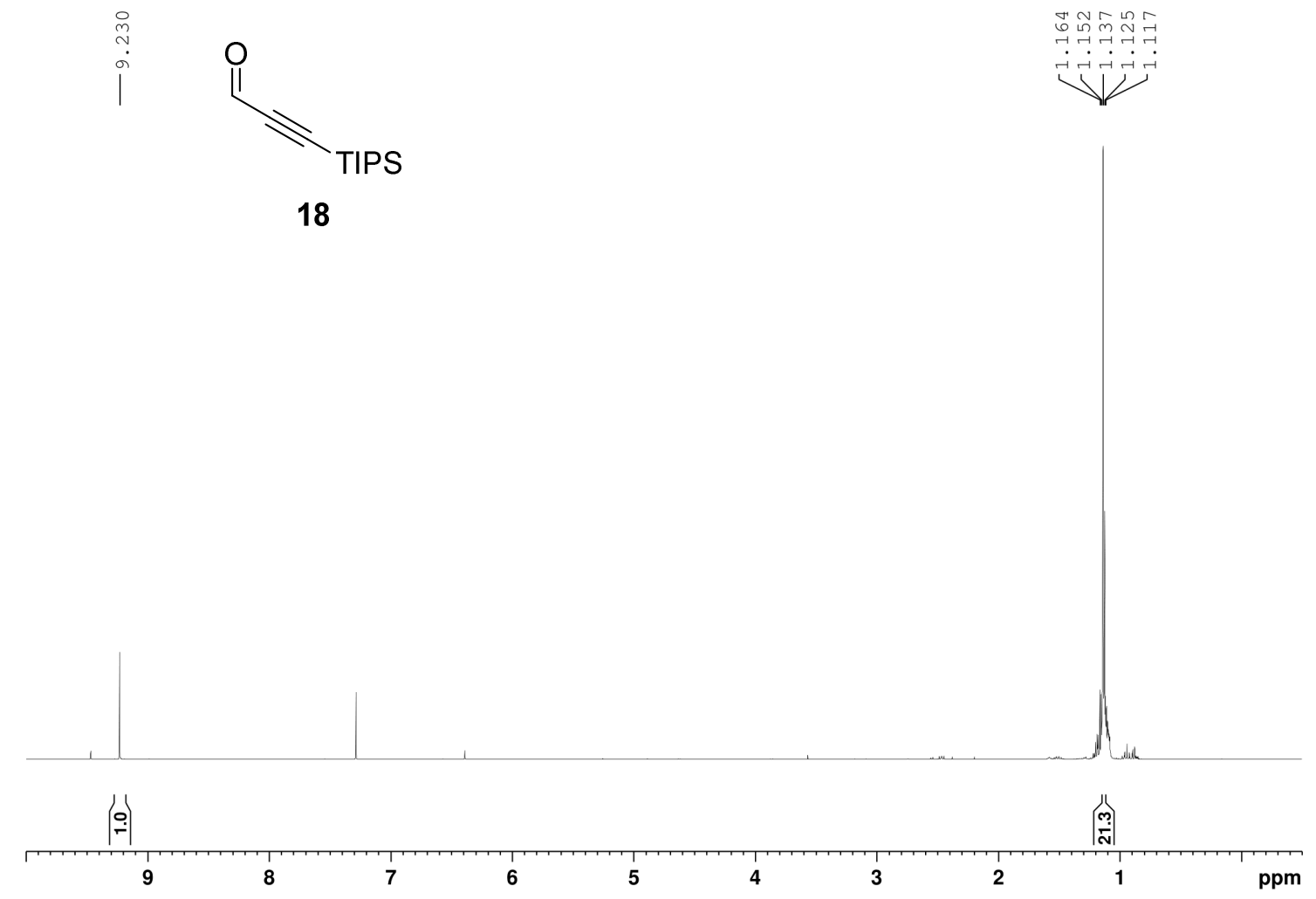

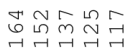



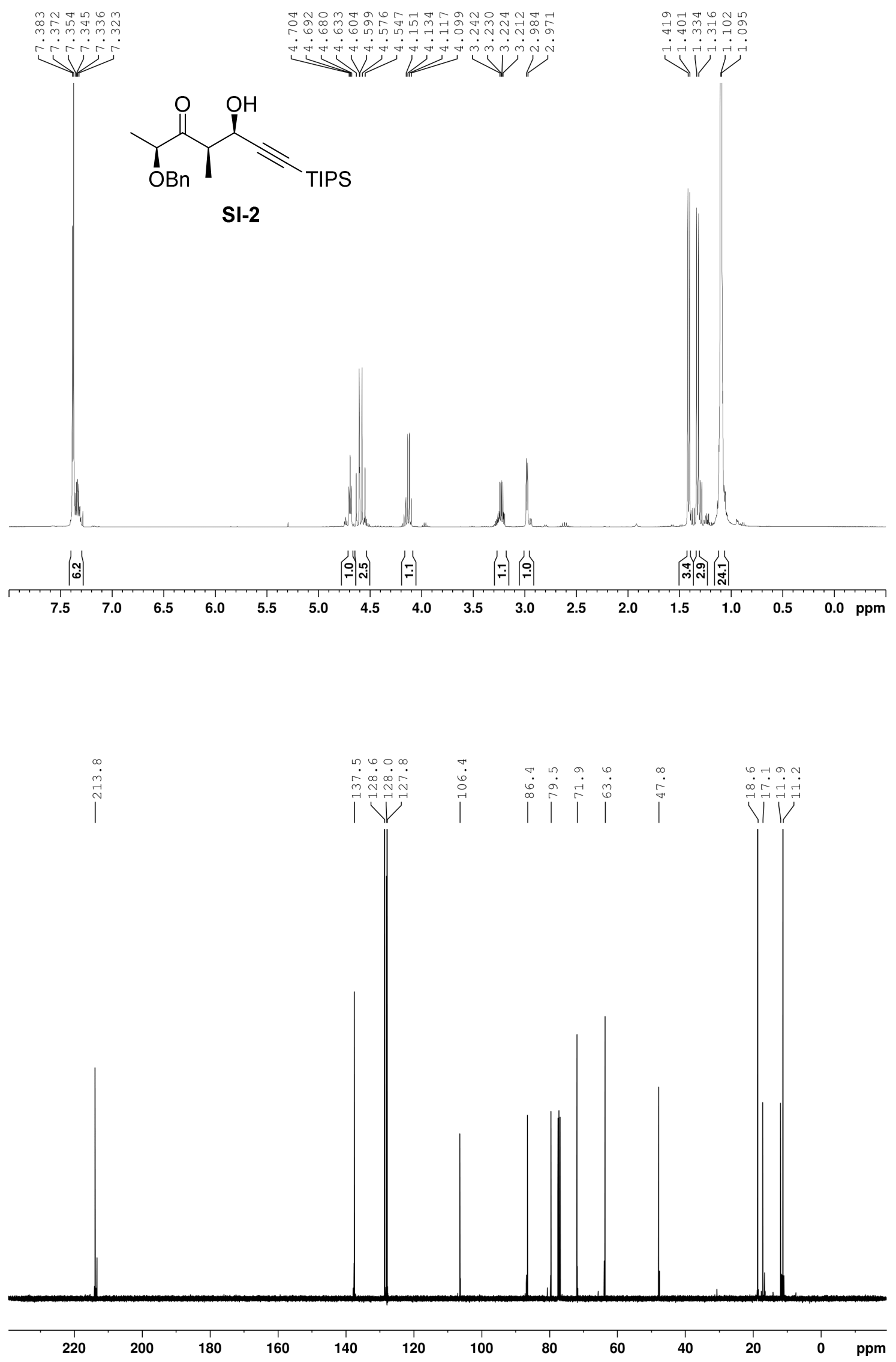

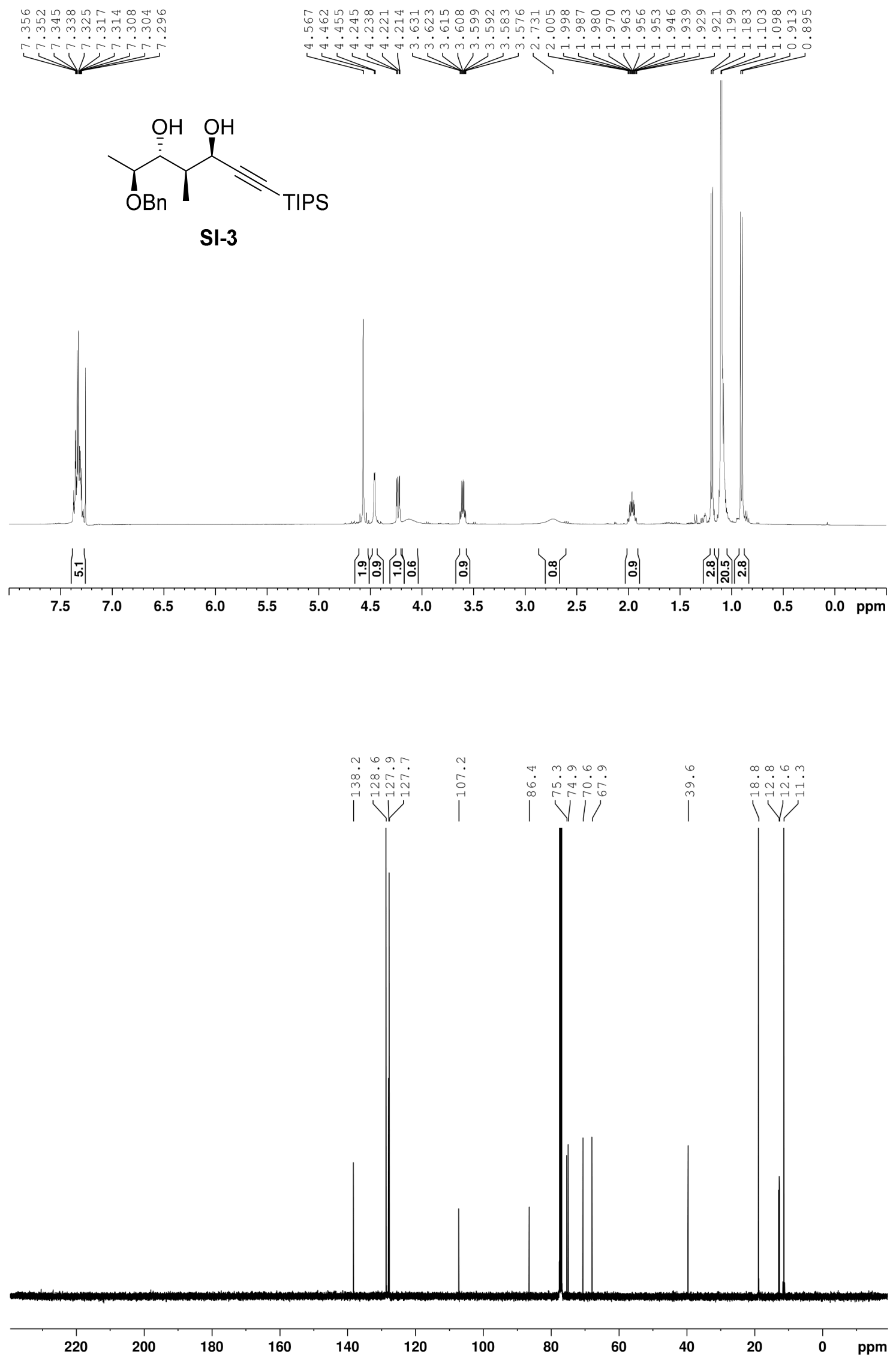

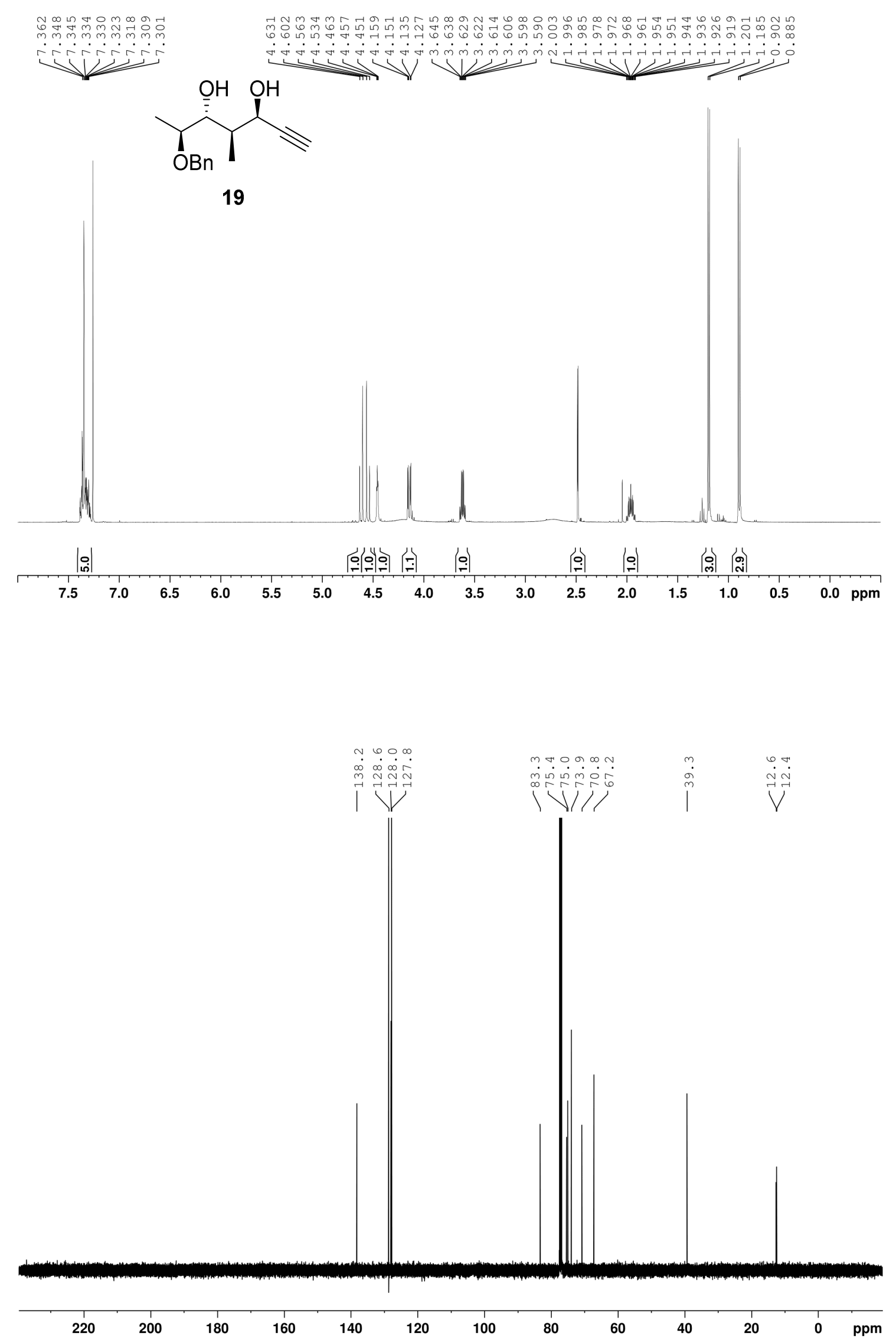

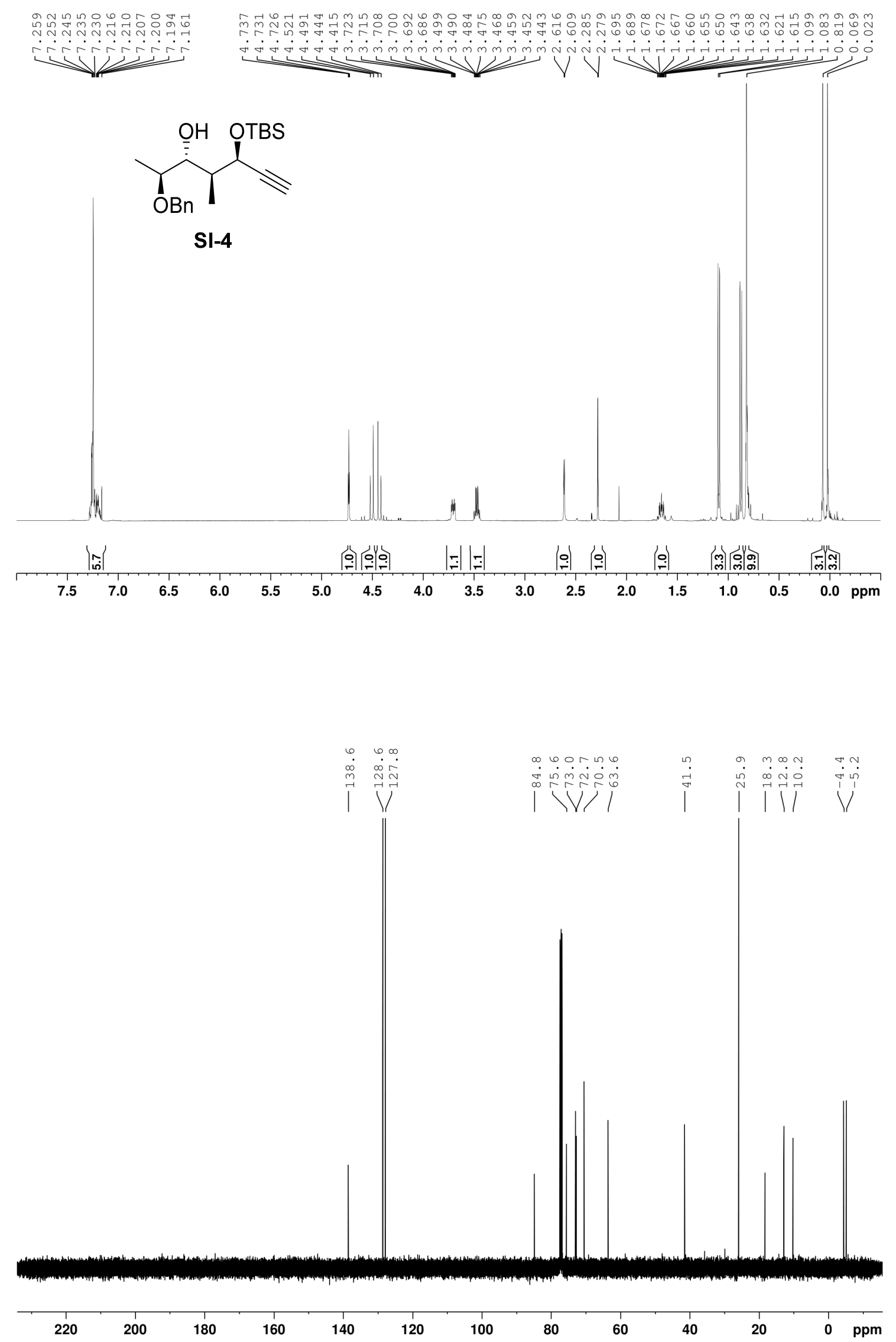

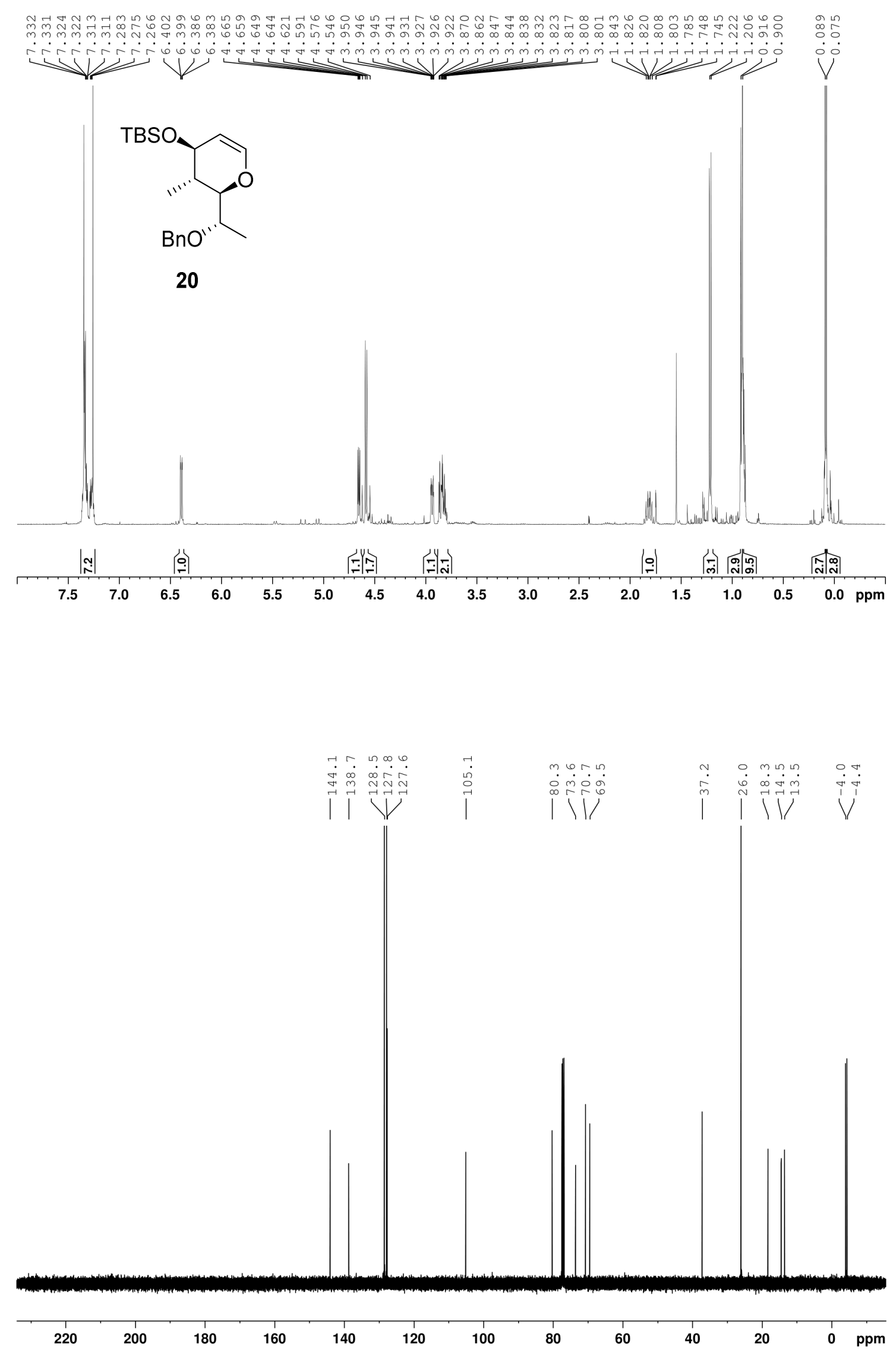


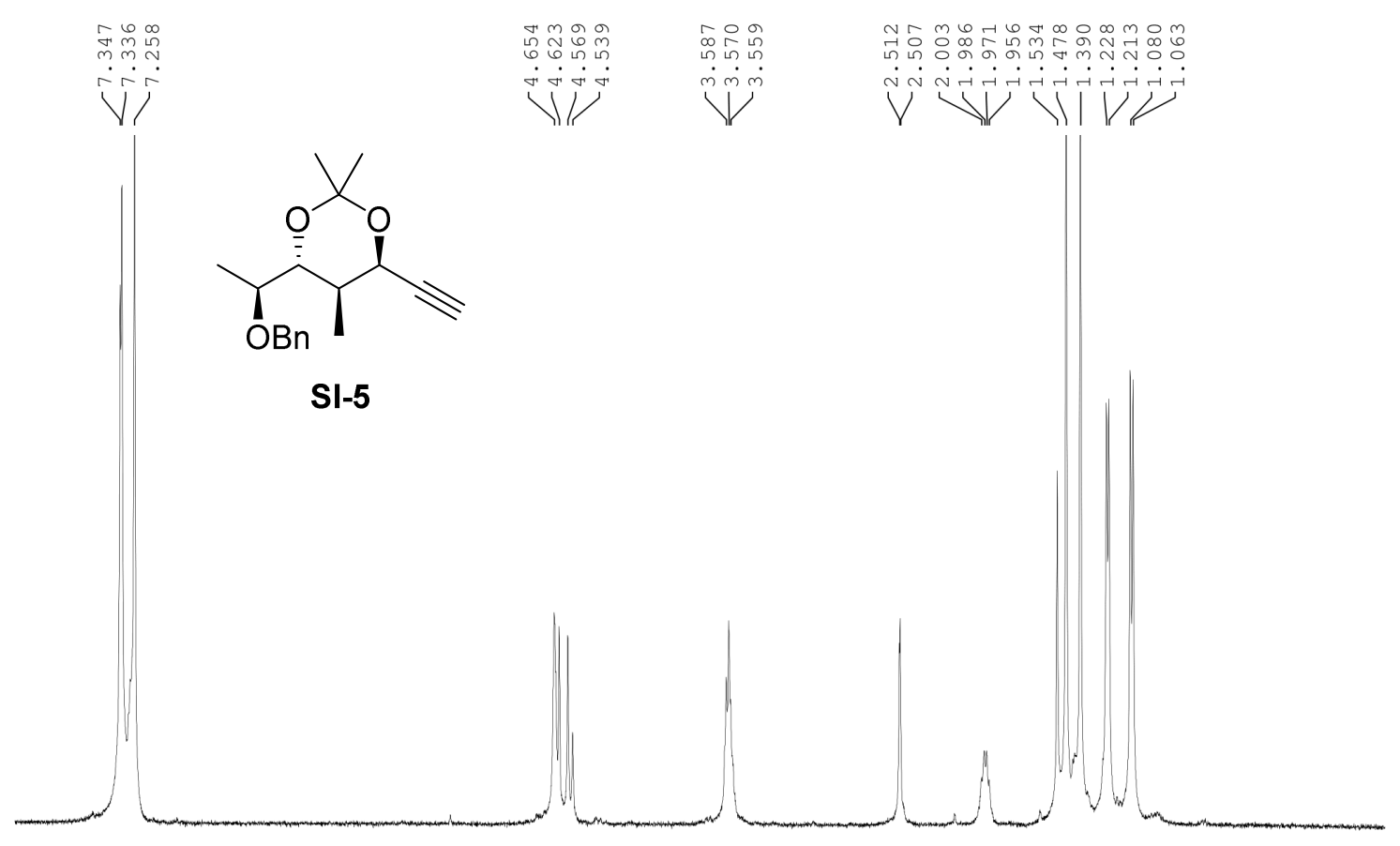

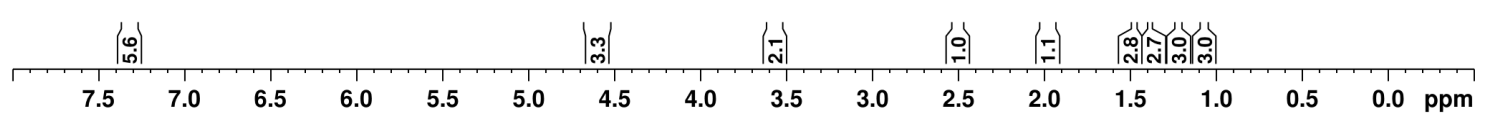

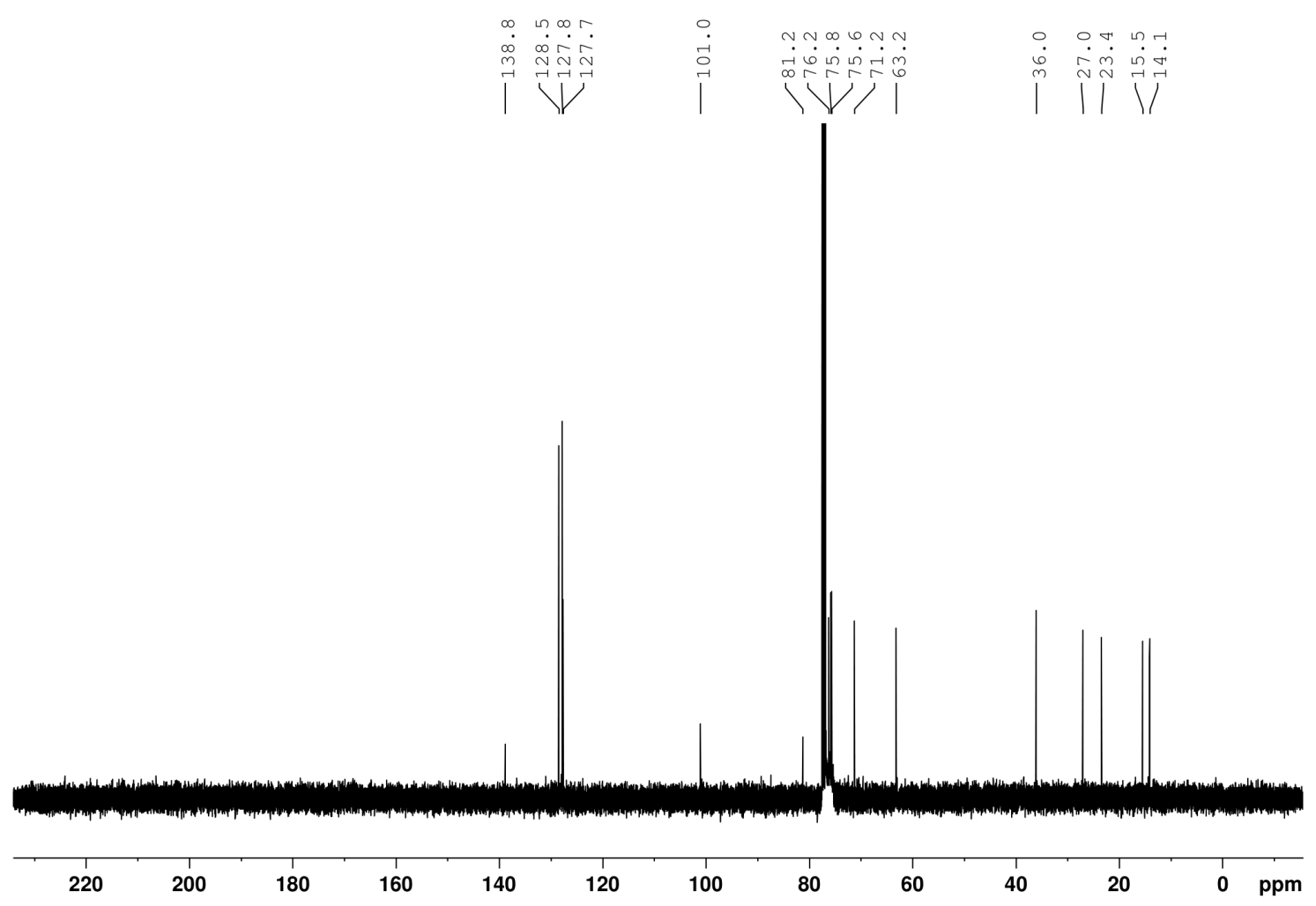



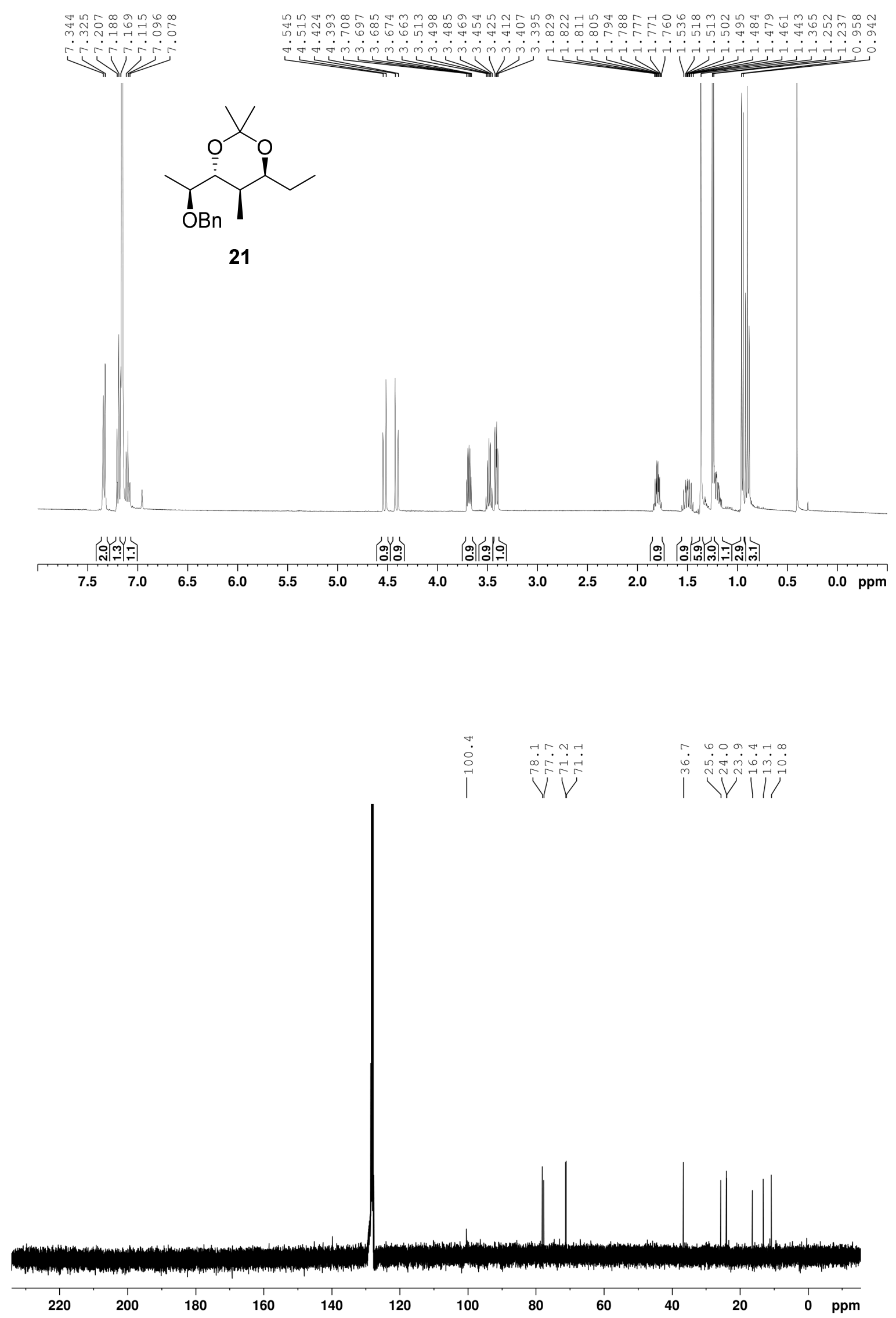


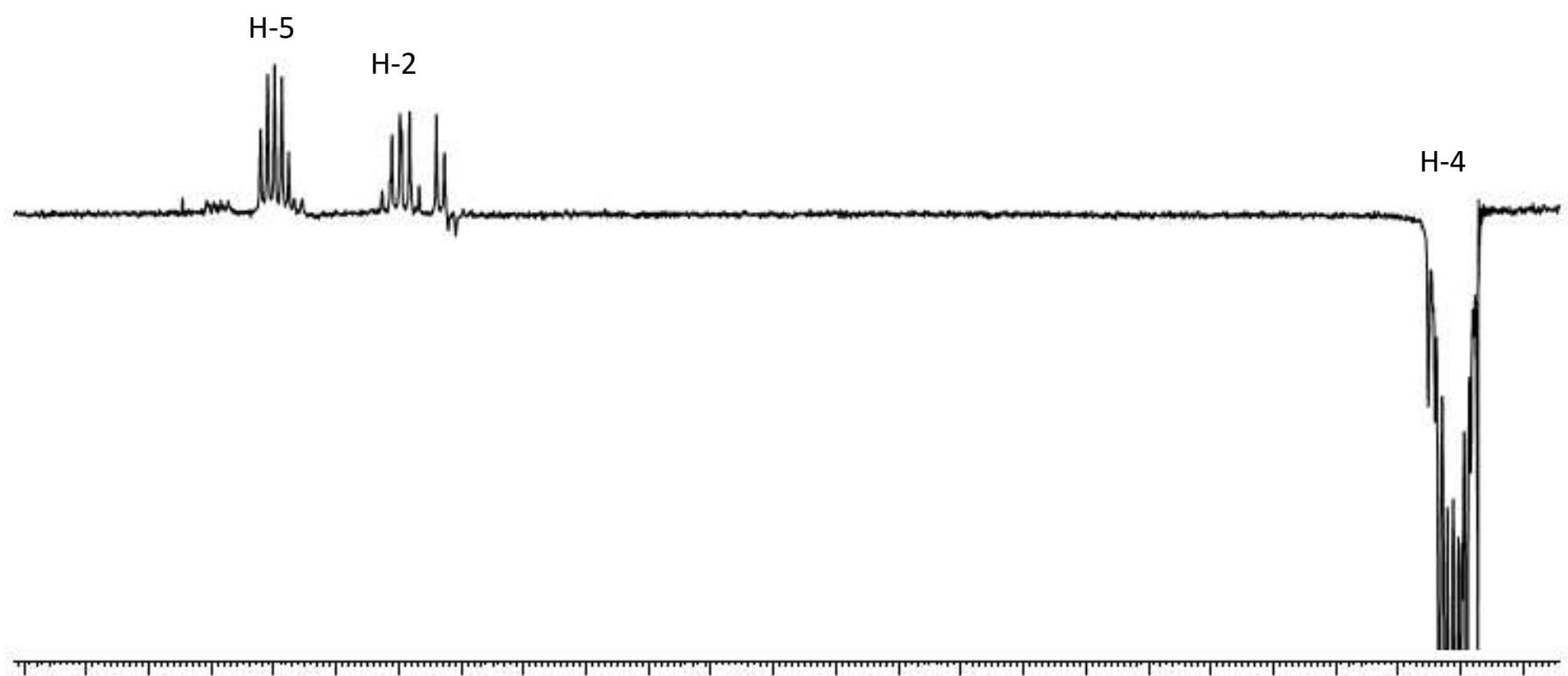

$\begin{array}{llllllllllllllllllllllll}4.1 & 4.0 & 3.9 & 3.8 & 3.7 & 3.6 & 3.5 & 3.4 & 3.3 & 3.2 & 3.1 & 3.0 & 2.9 & 2.8 & 2.7 & 2.6 & 2.5 & 2.4 & 2.3 & 2.2 & 2.1 & 2.0 & 1.9 & \mathrm{ppm}\end{array}$

H-3

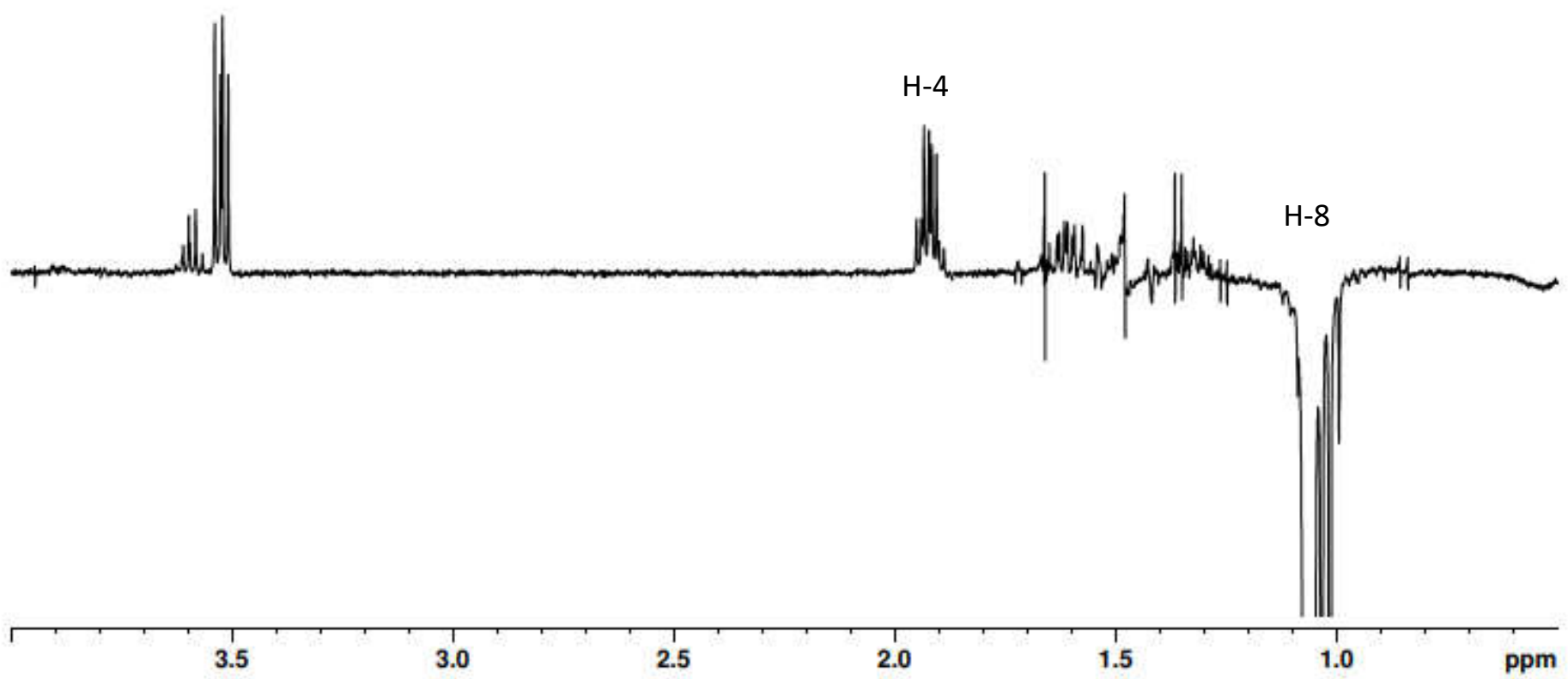



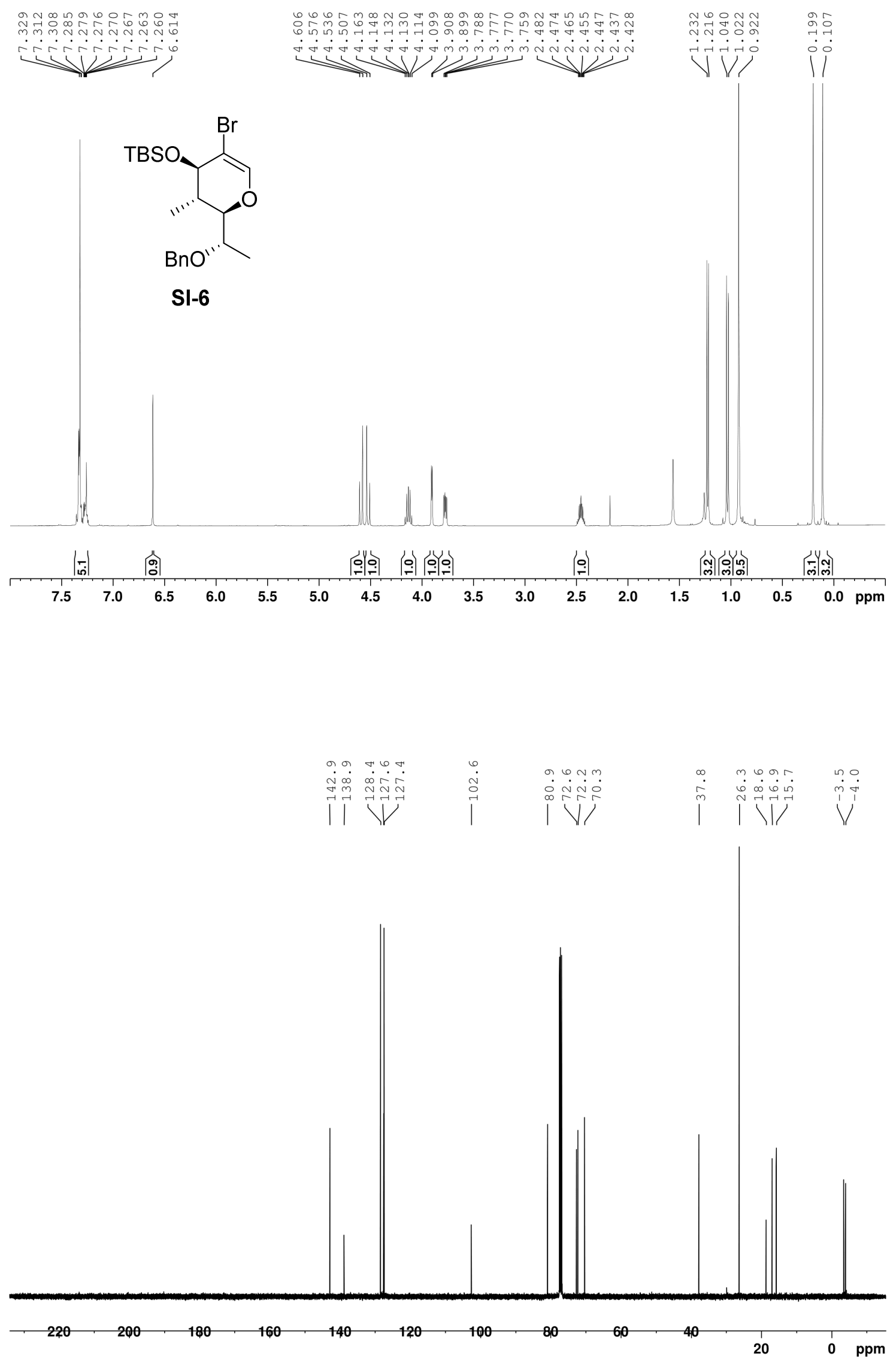

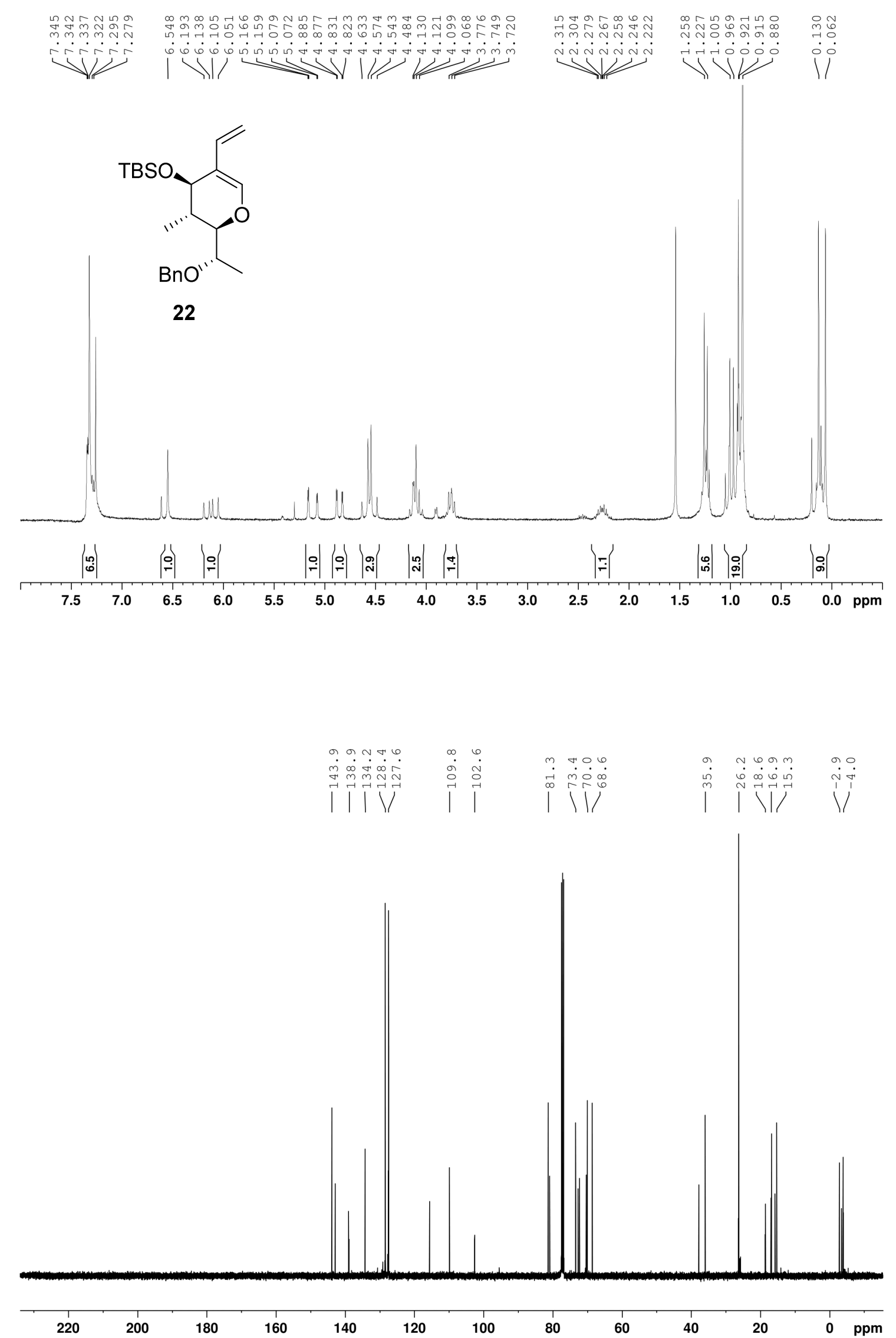


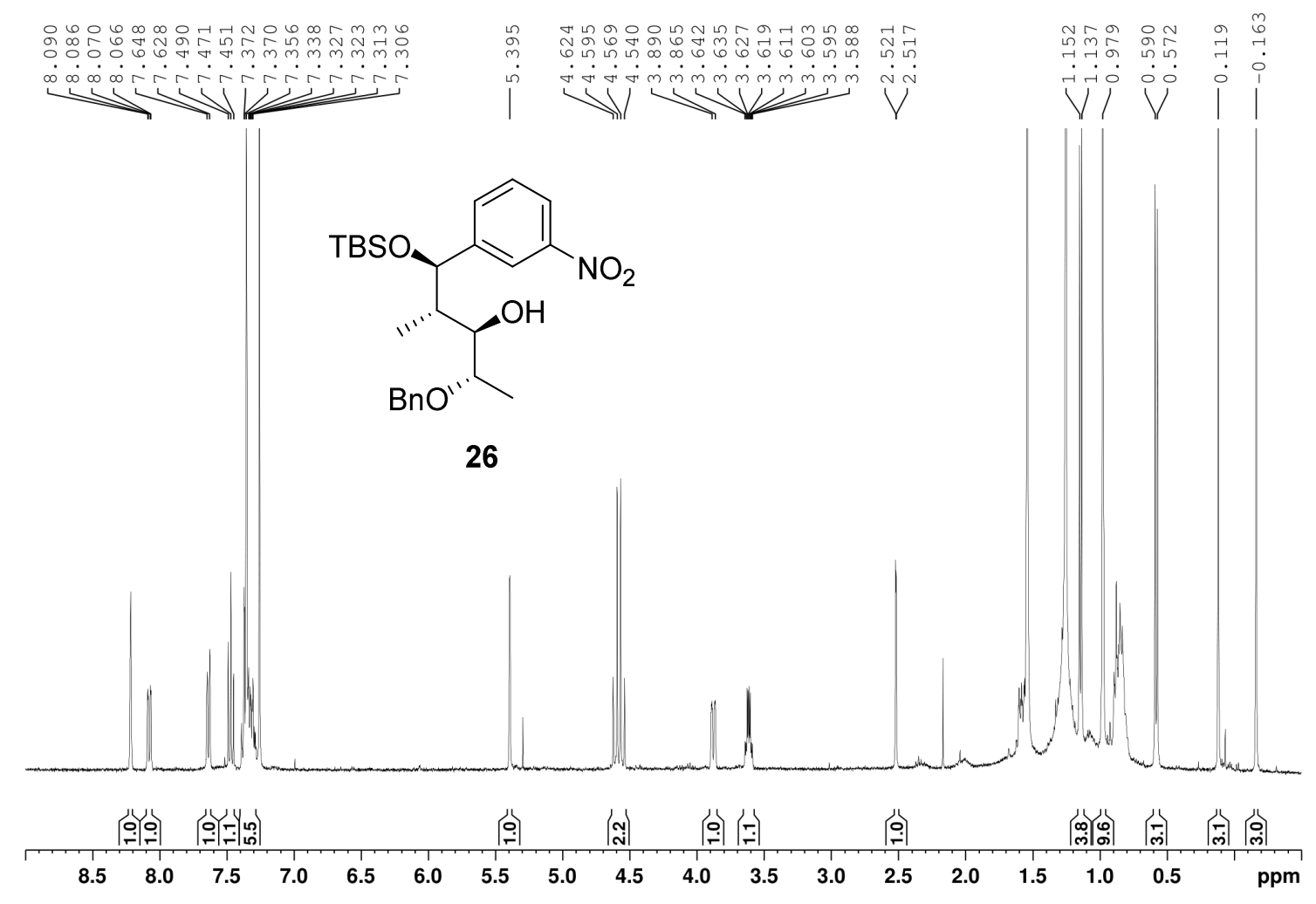



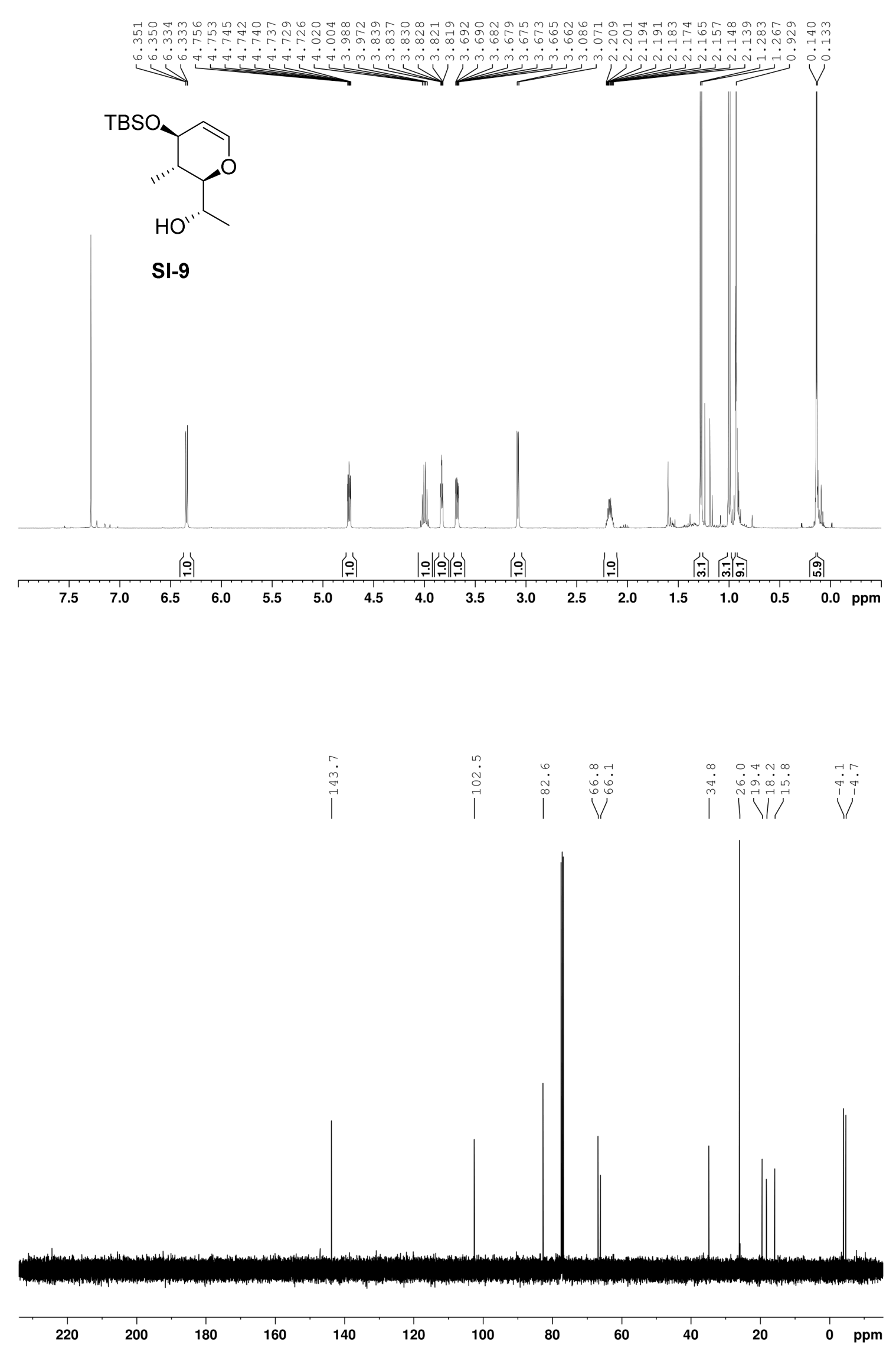

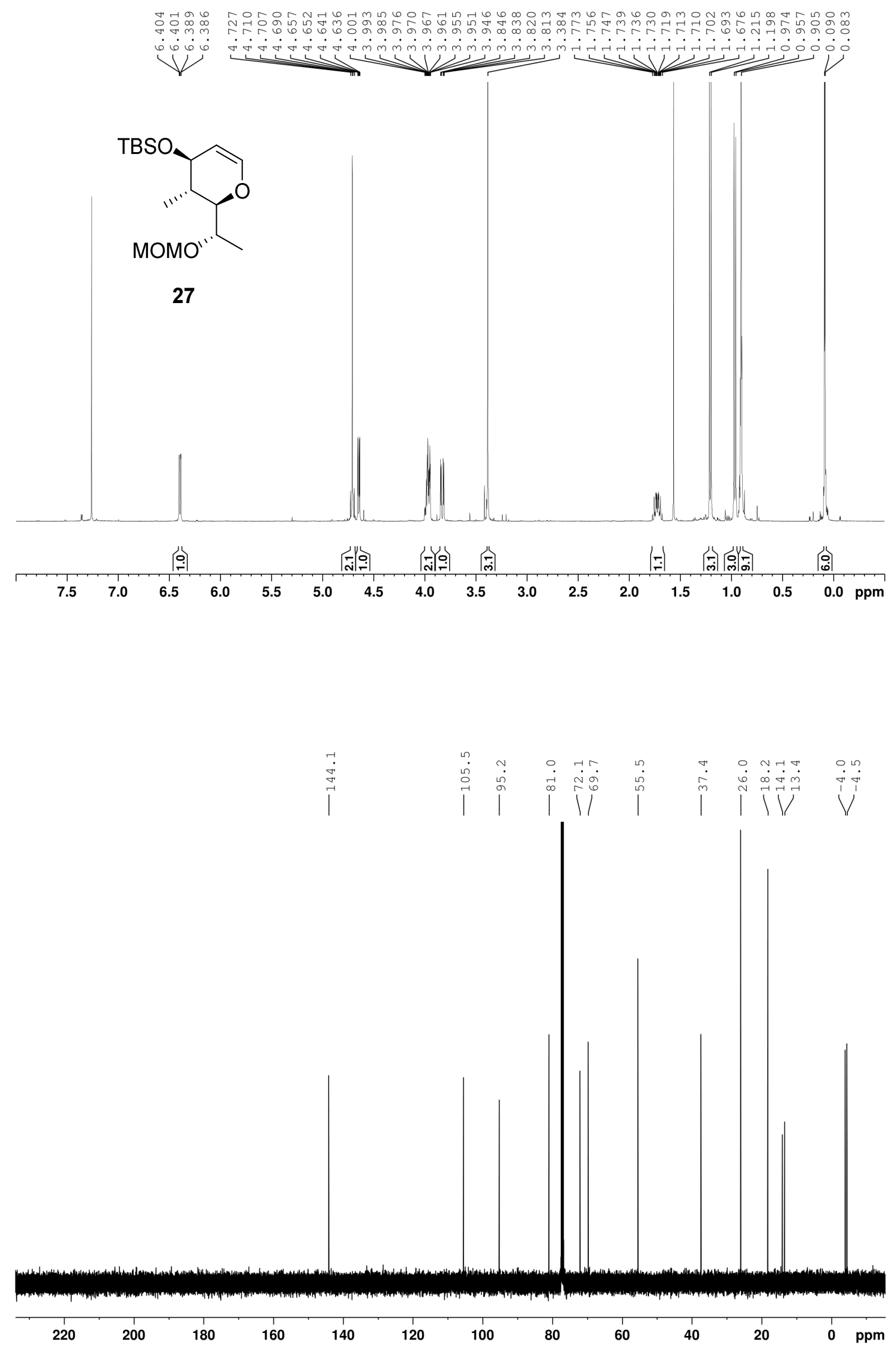

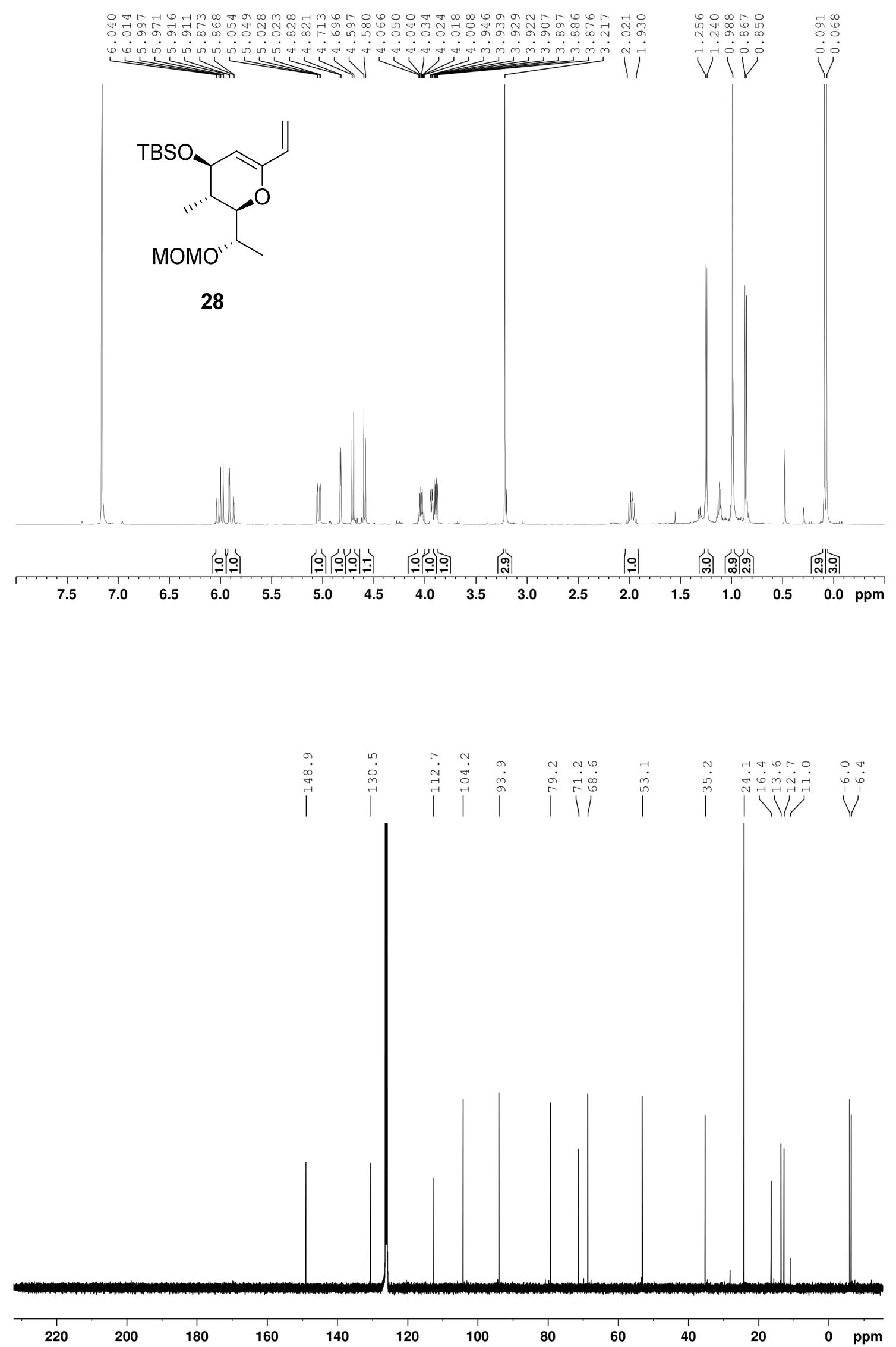

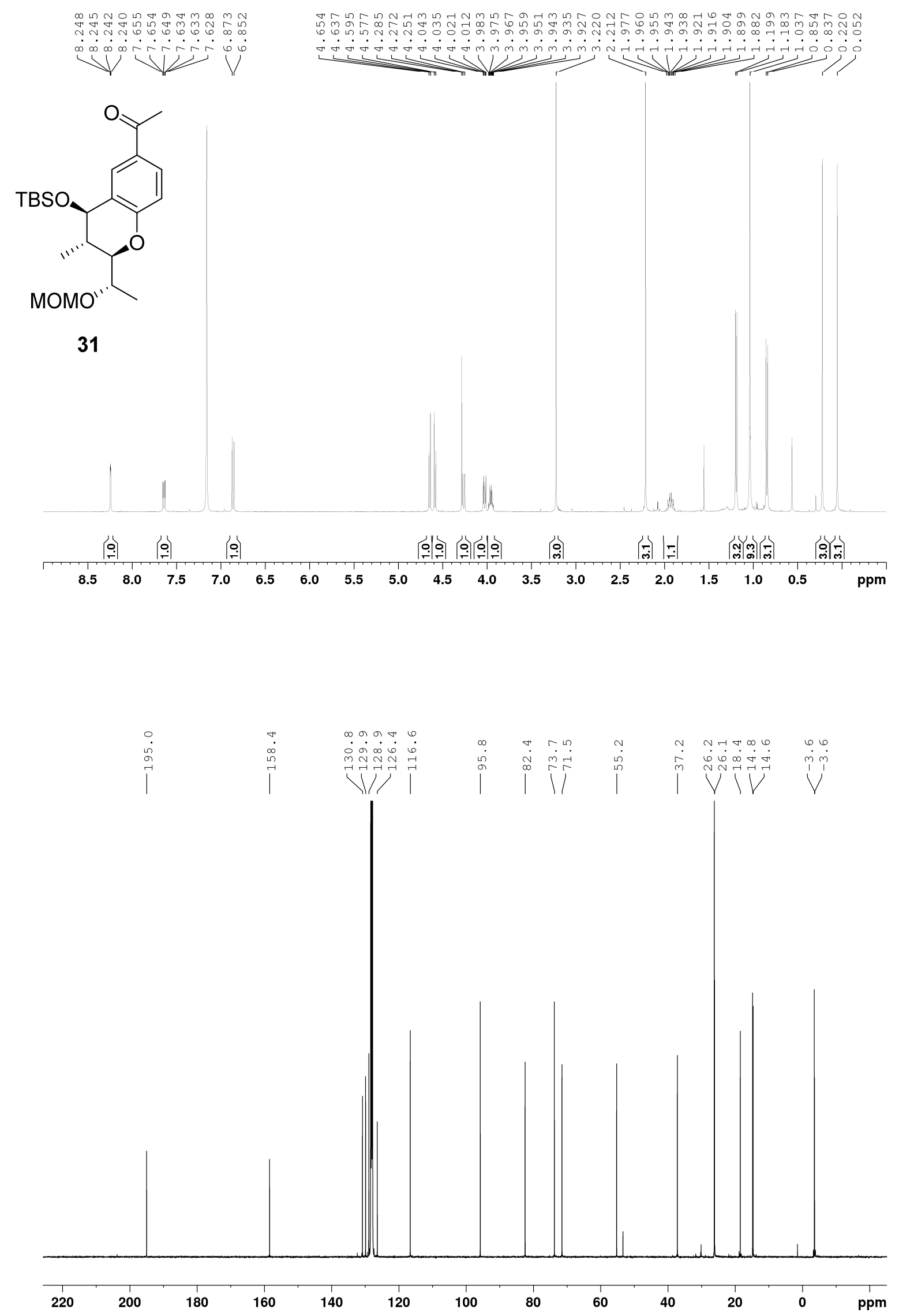


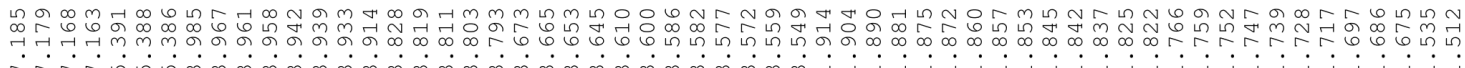

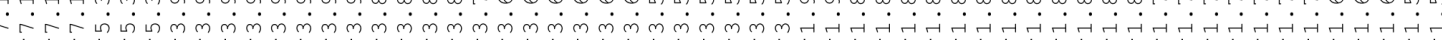
$\rightarrow$ 1III)

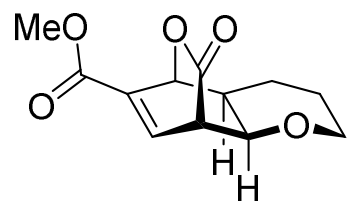
exo-35a
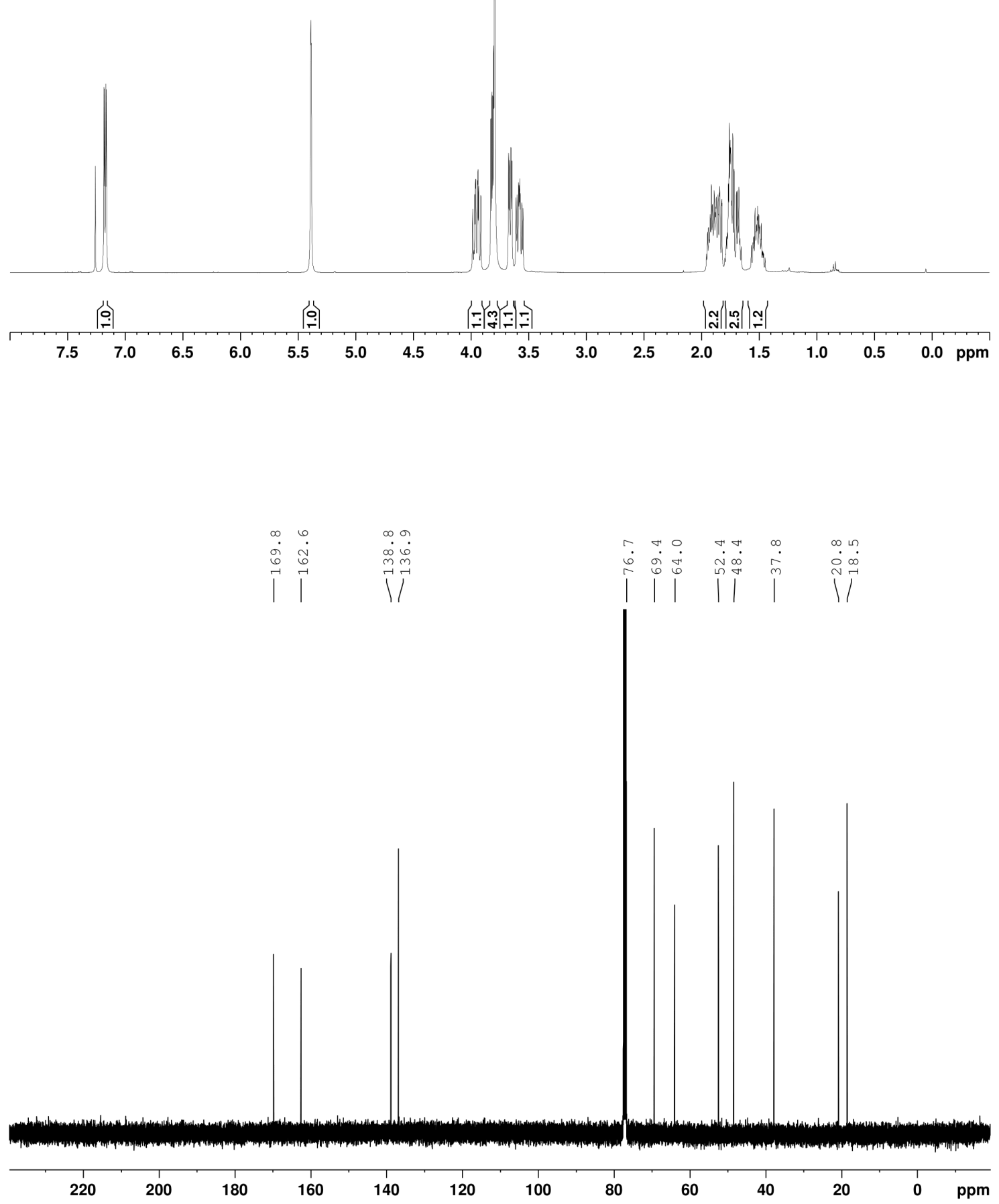


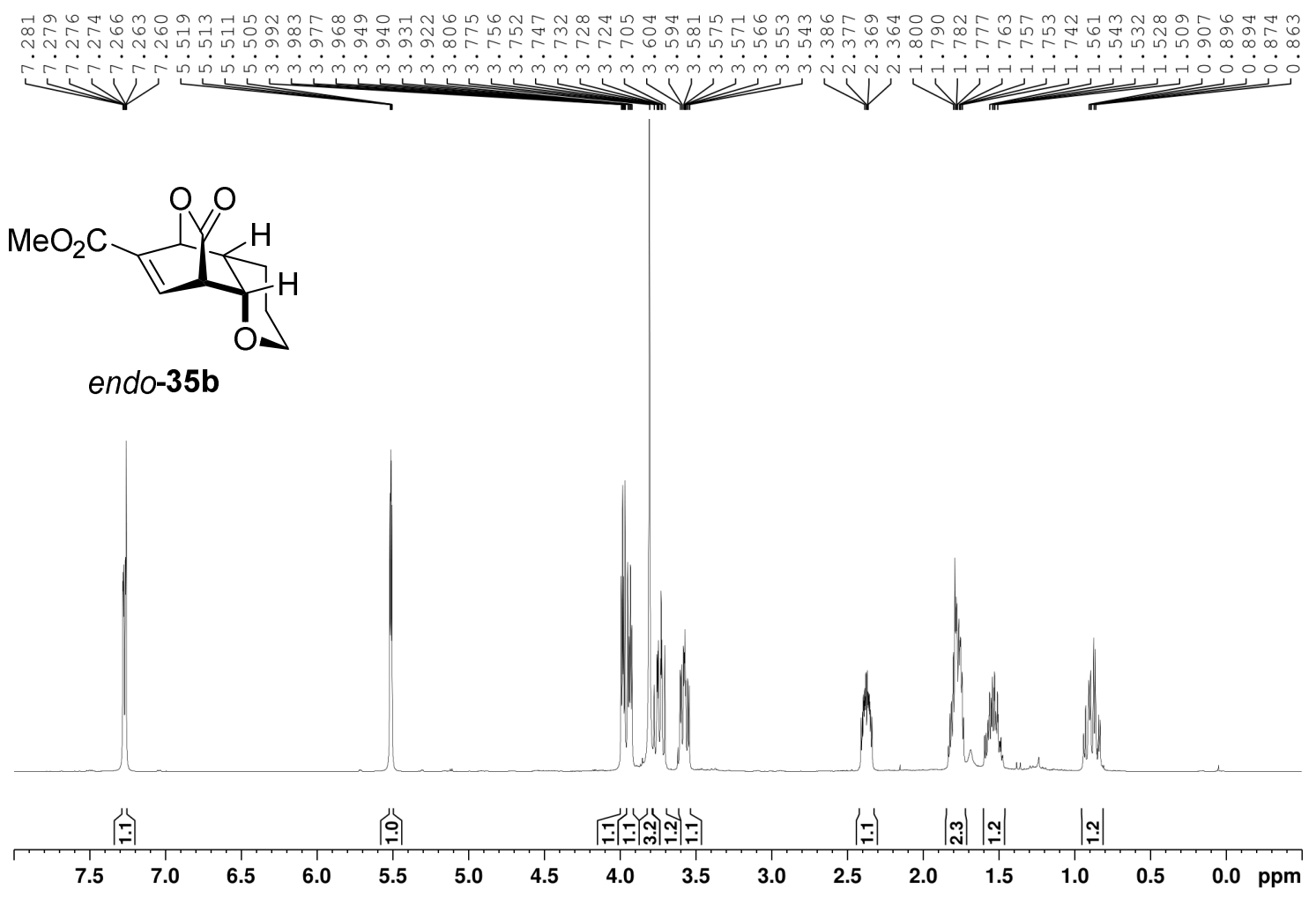




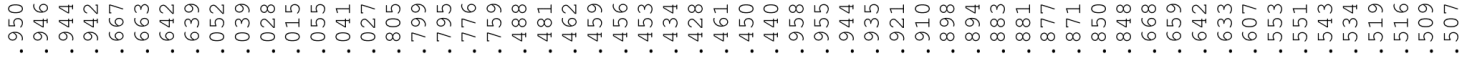

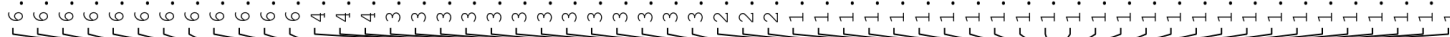<smiles>COC(=O)C1=CC2CCCOC2C=C1</smiles>

36

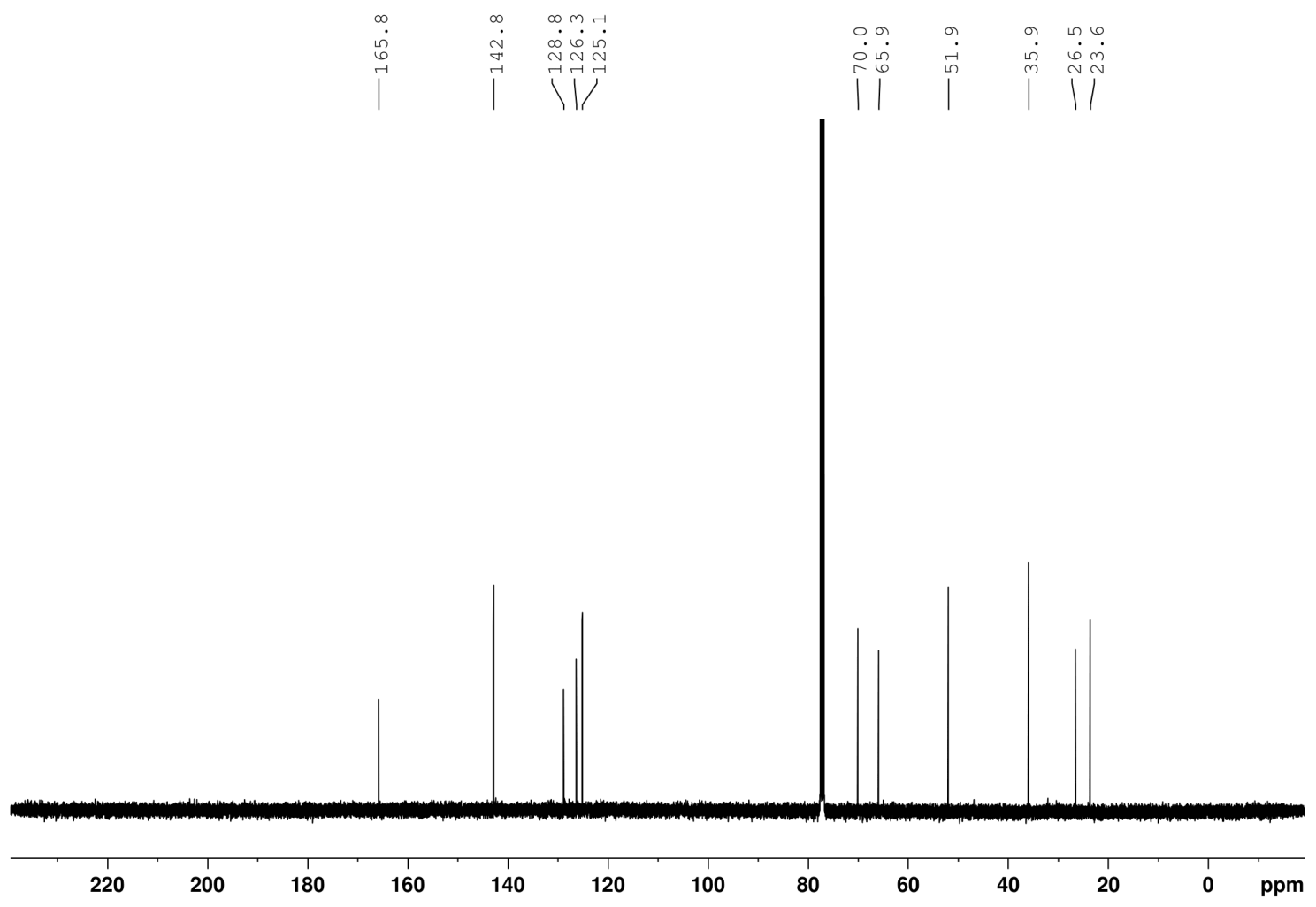



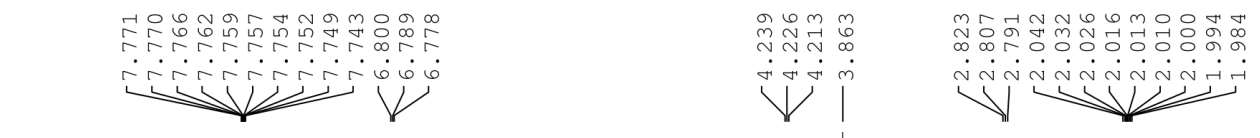<smiles>CC(=O)c1ccc2c(c1)CCCO2</smiles>

37

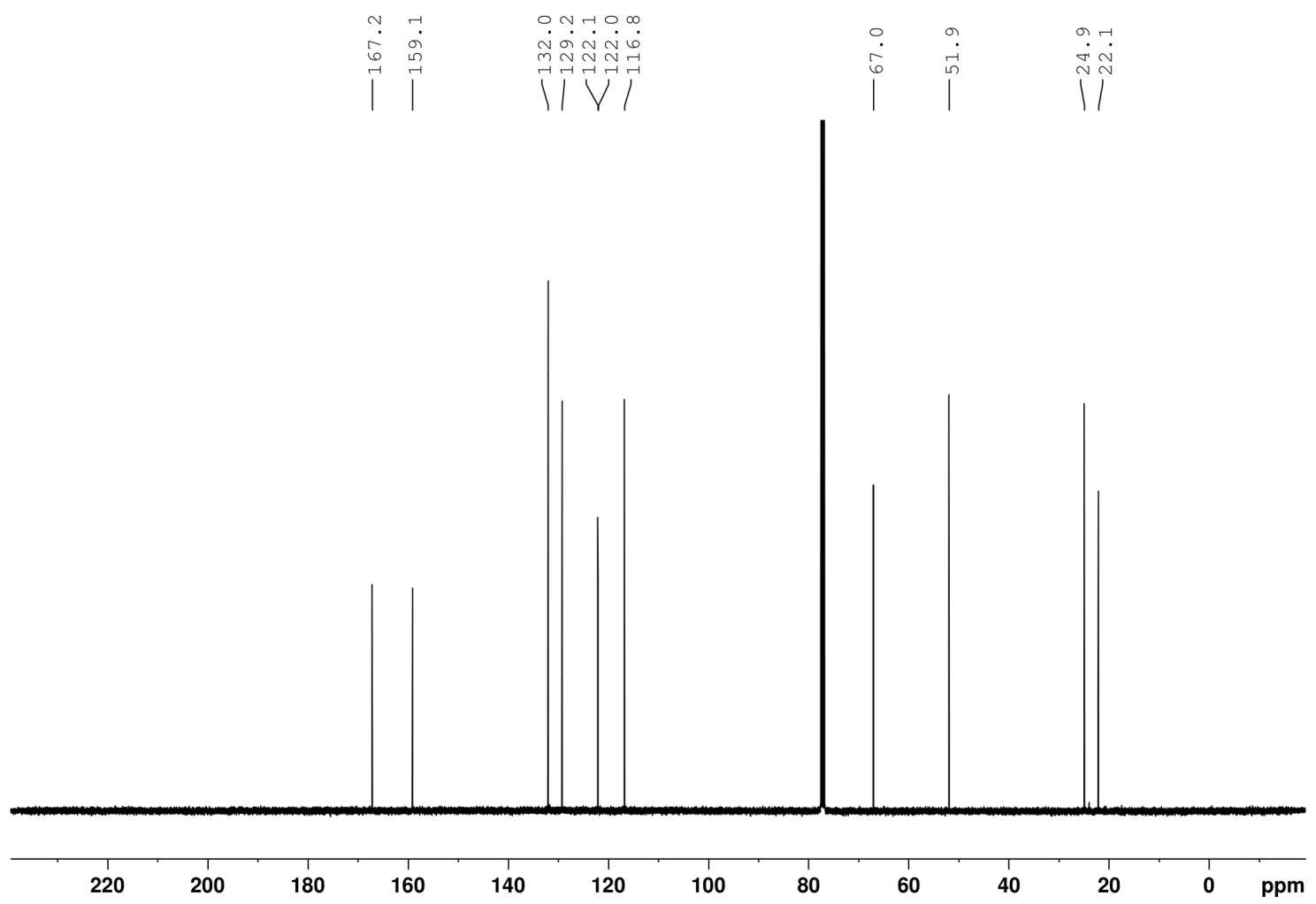



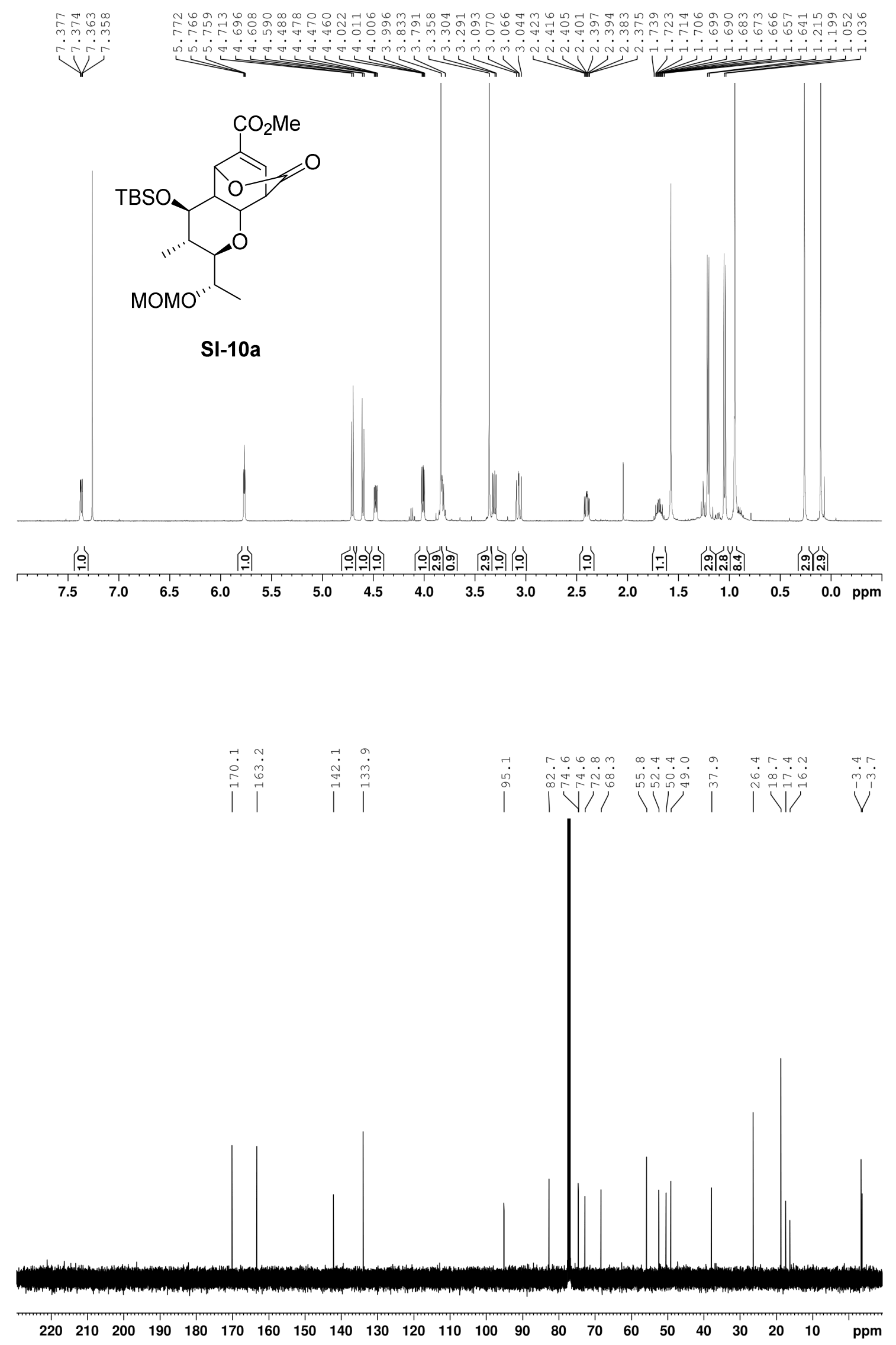


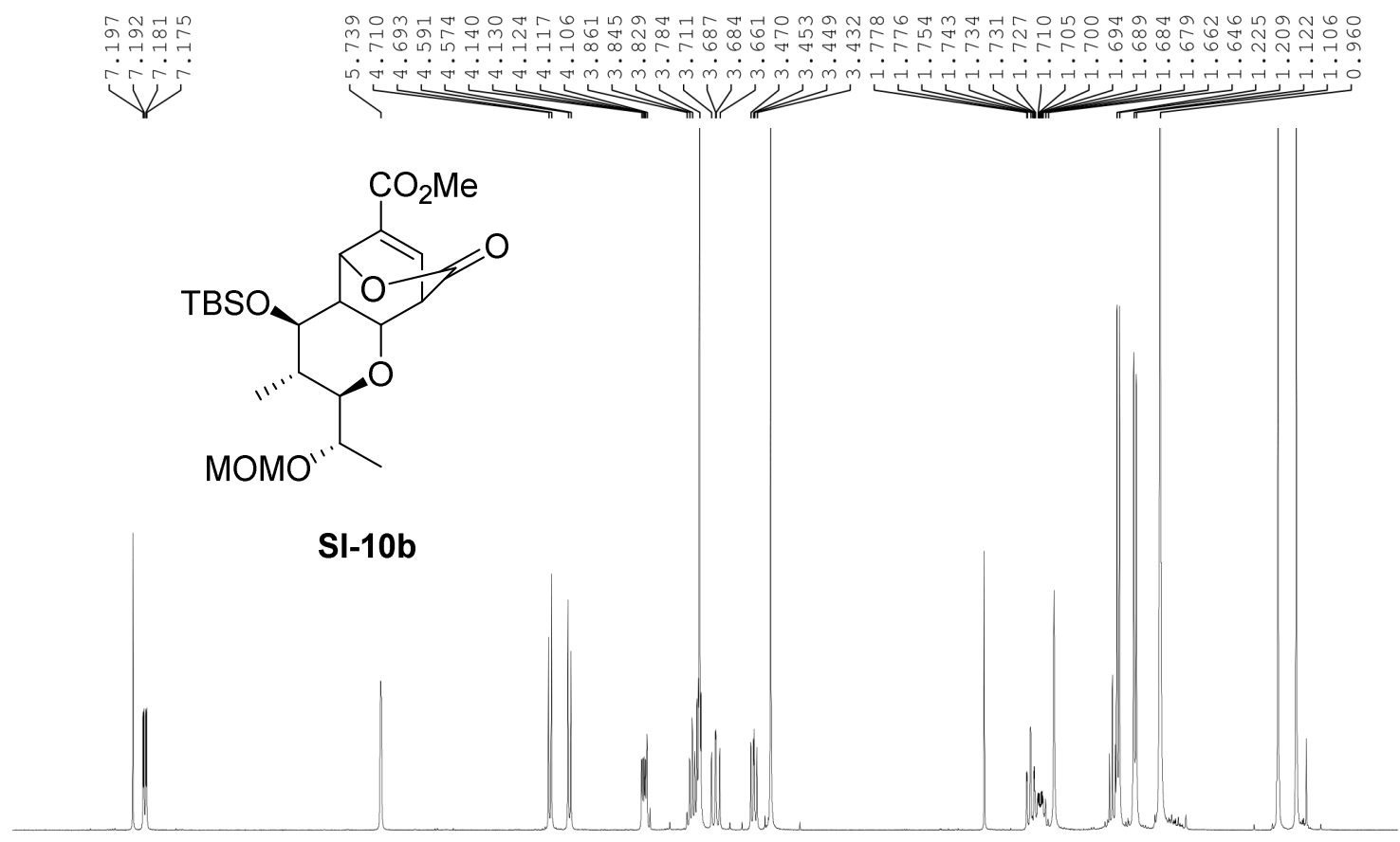

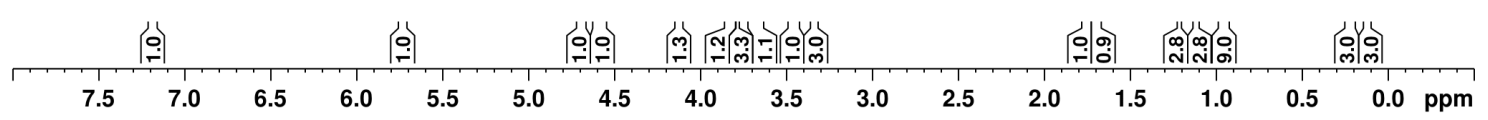

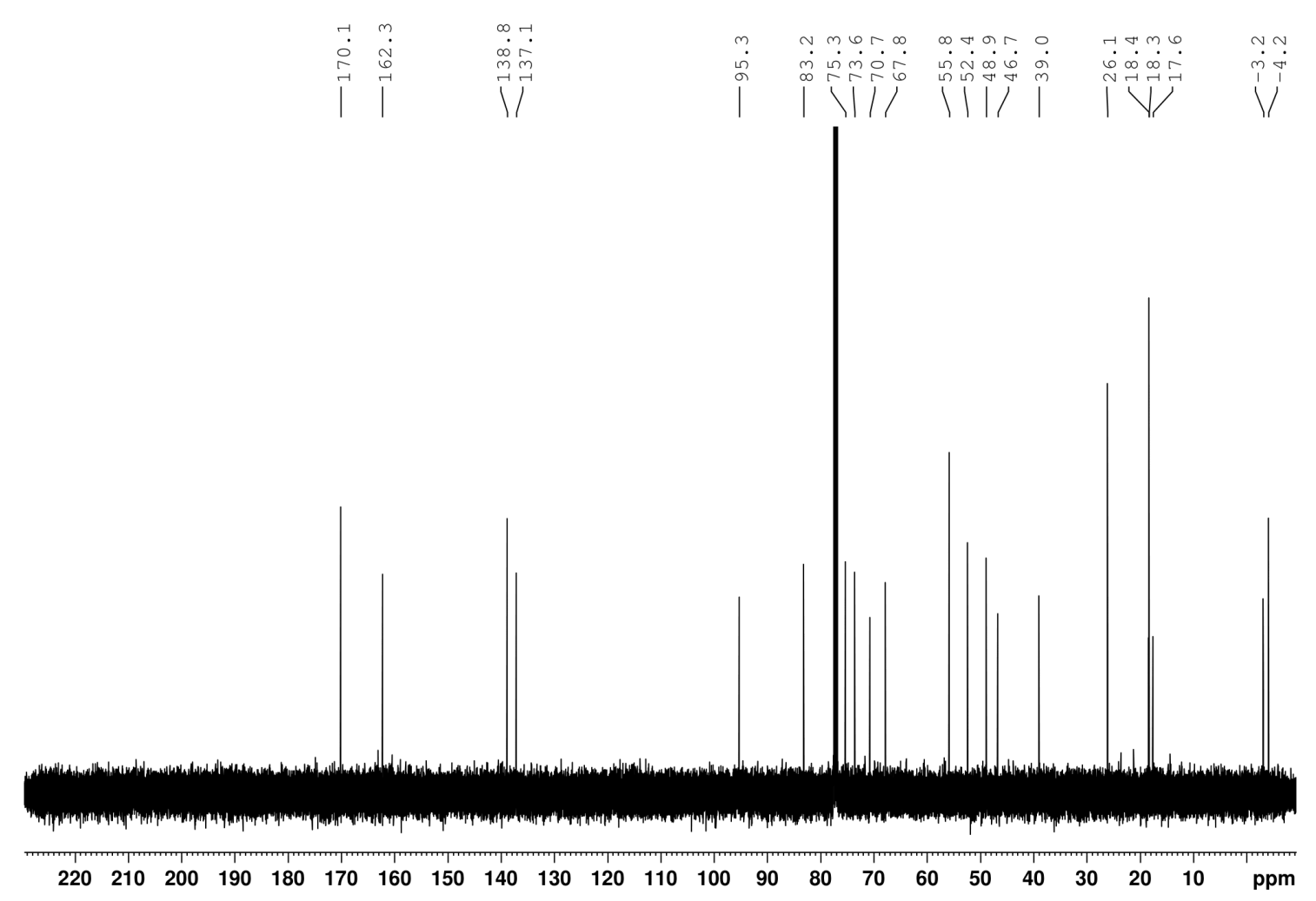



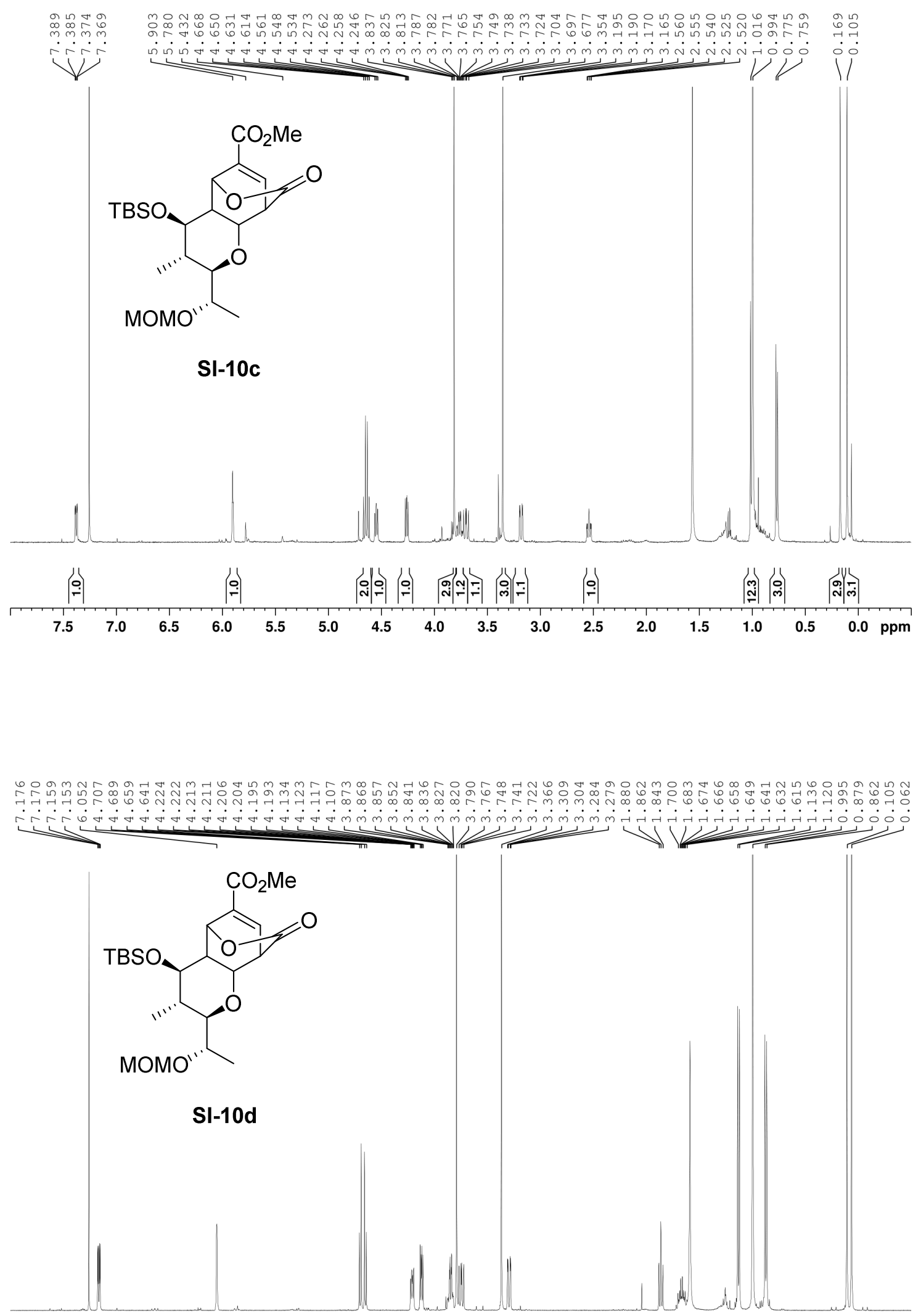

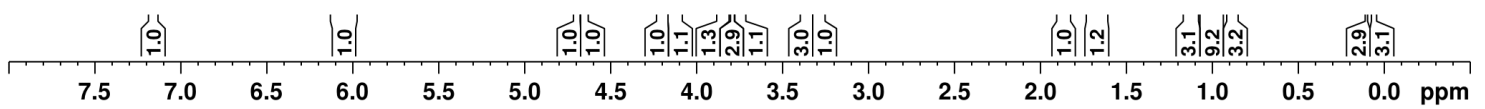




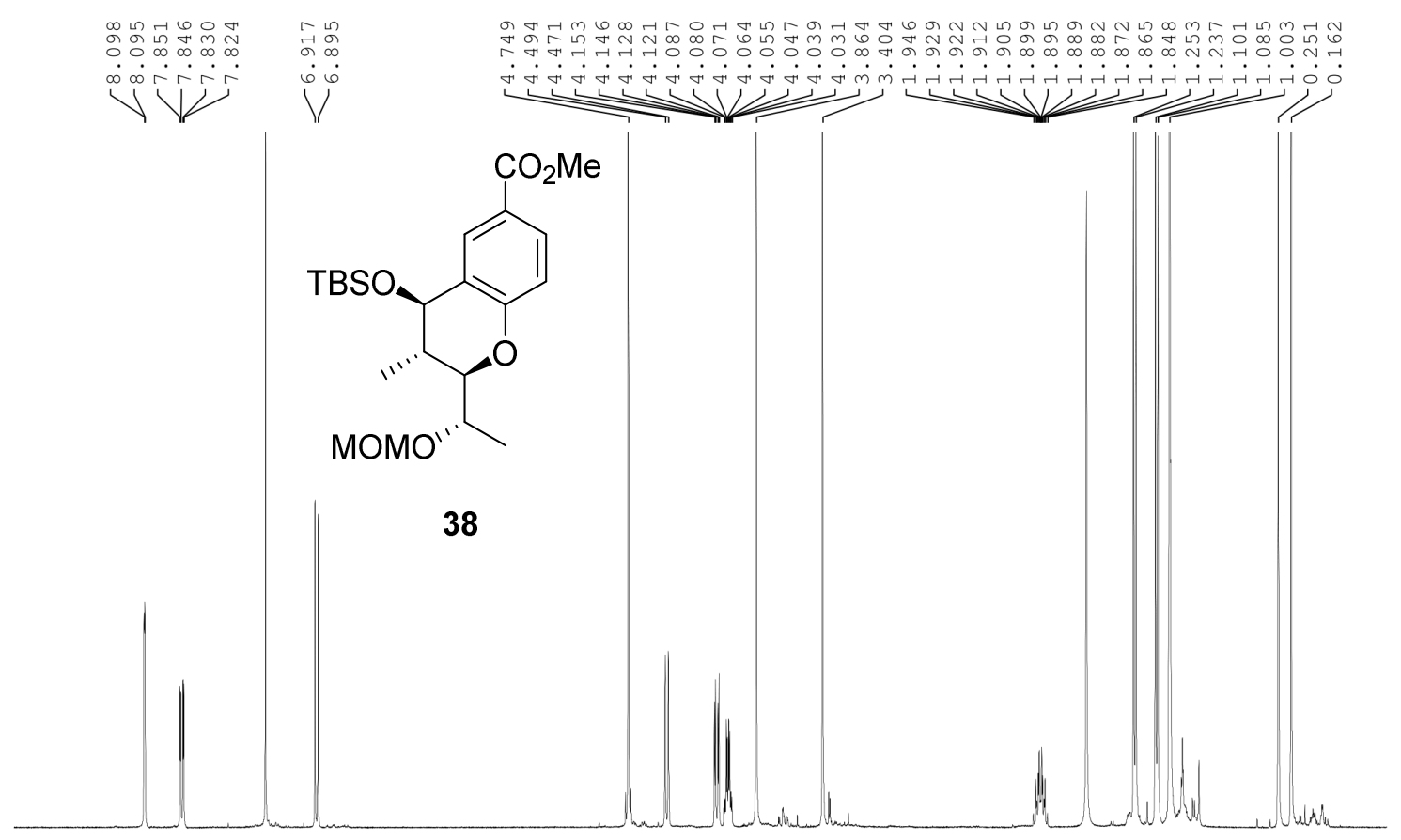

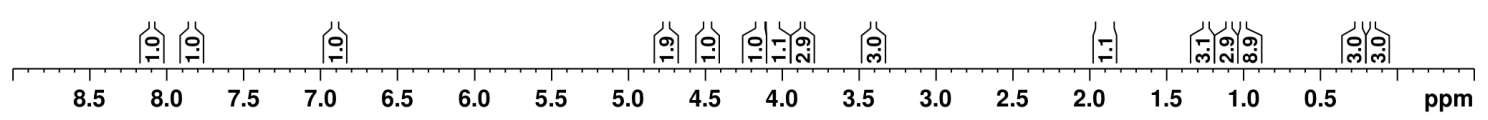

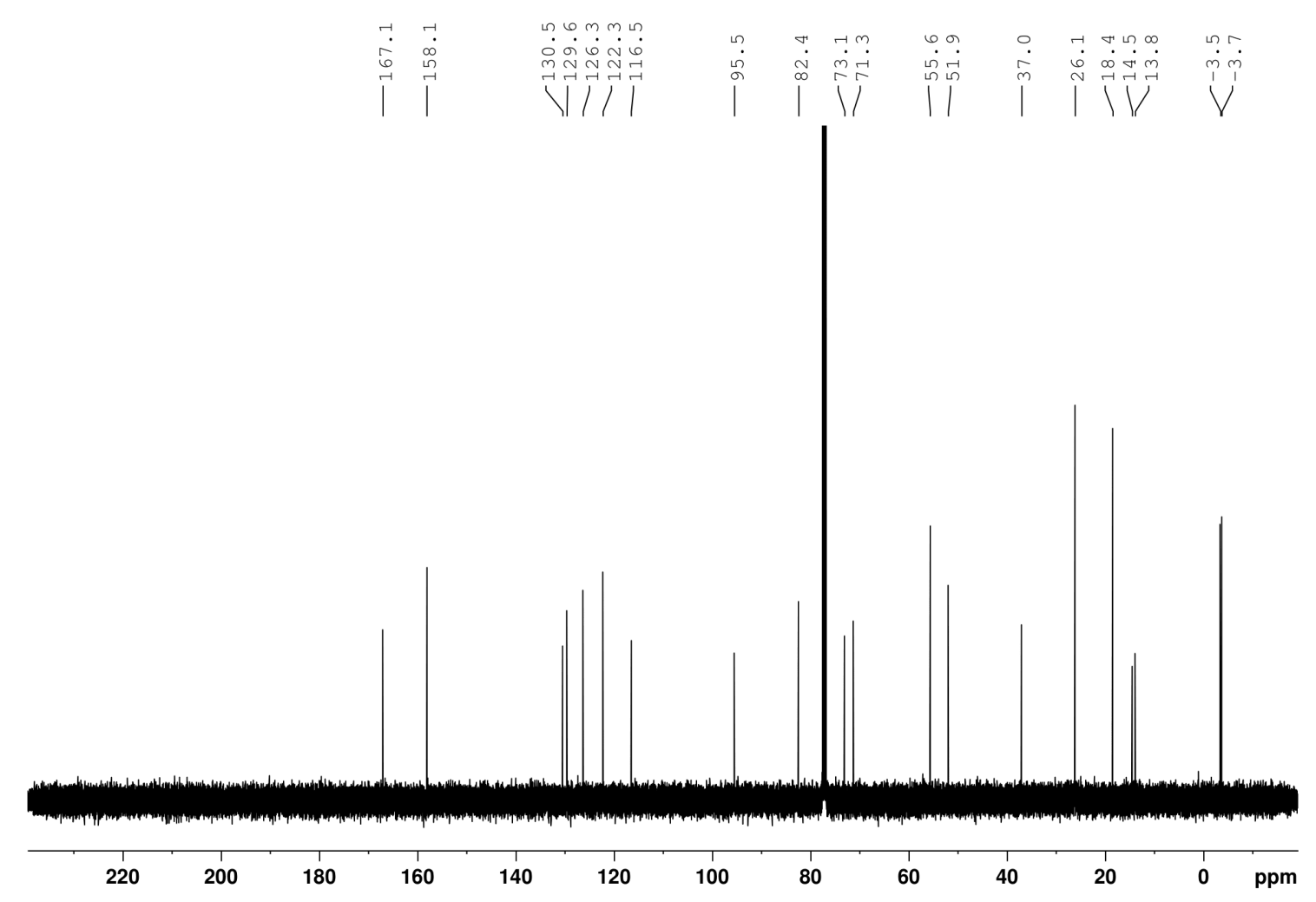



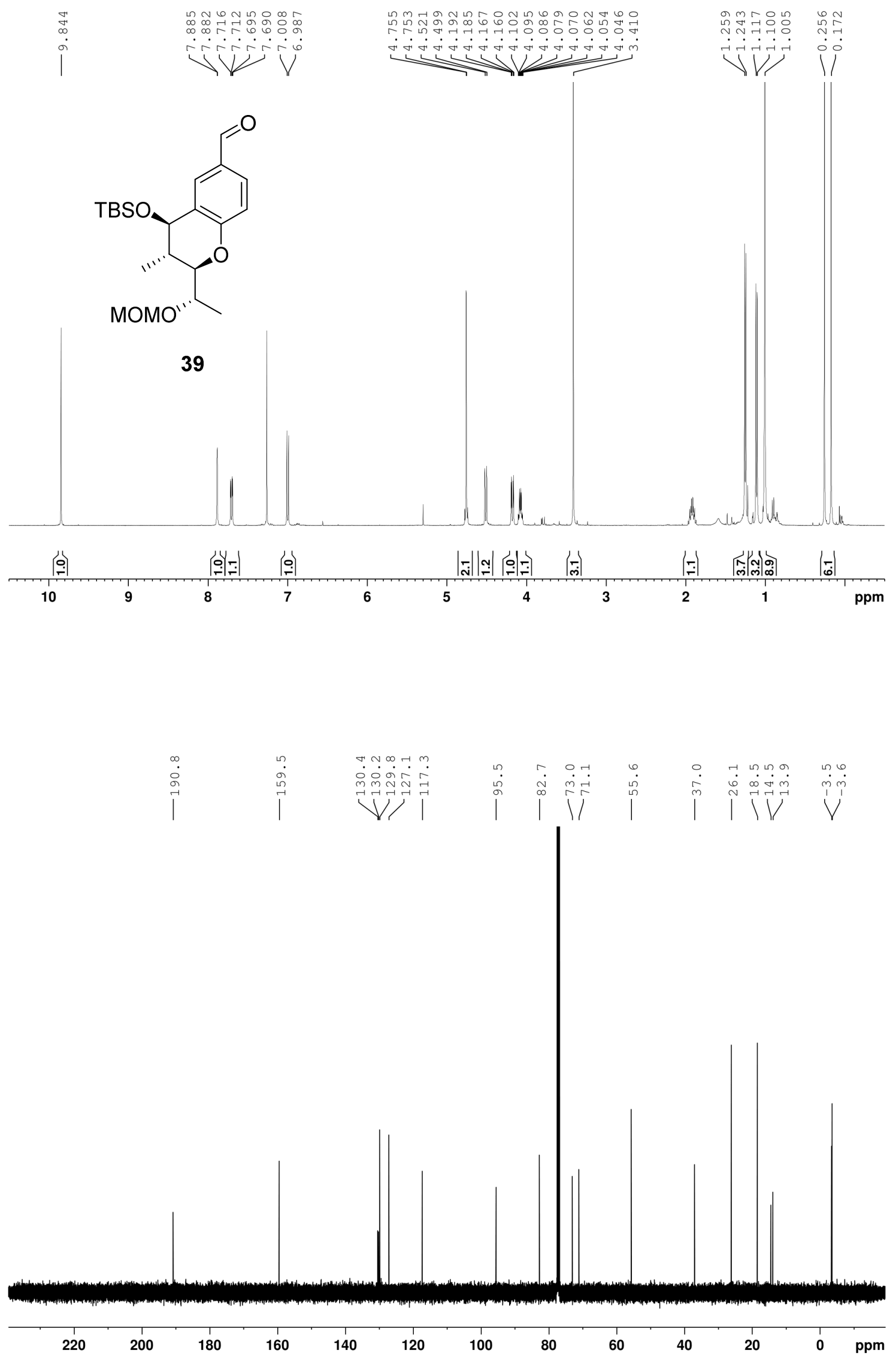

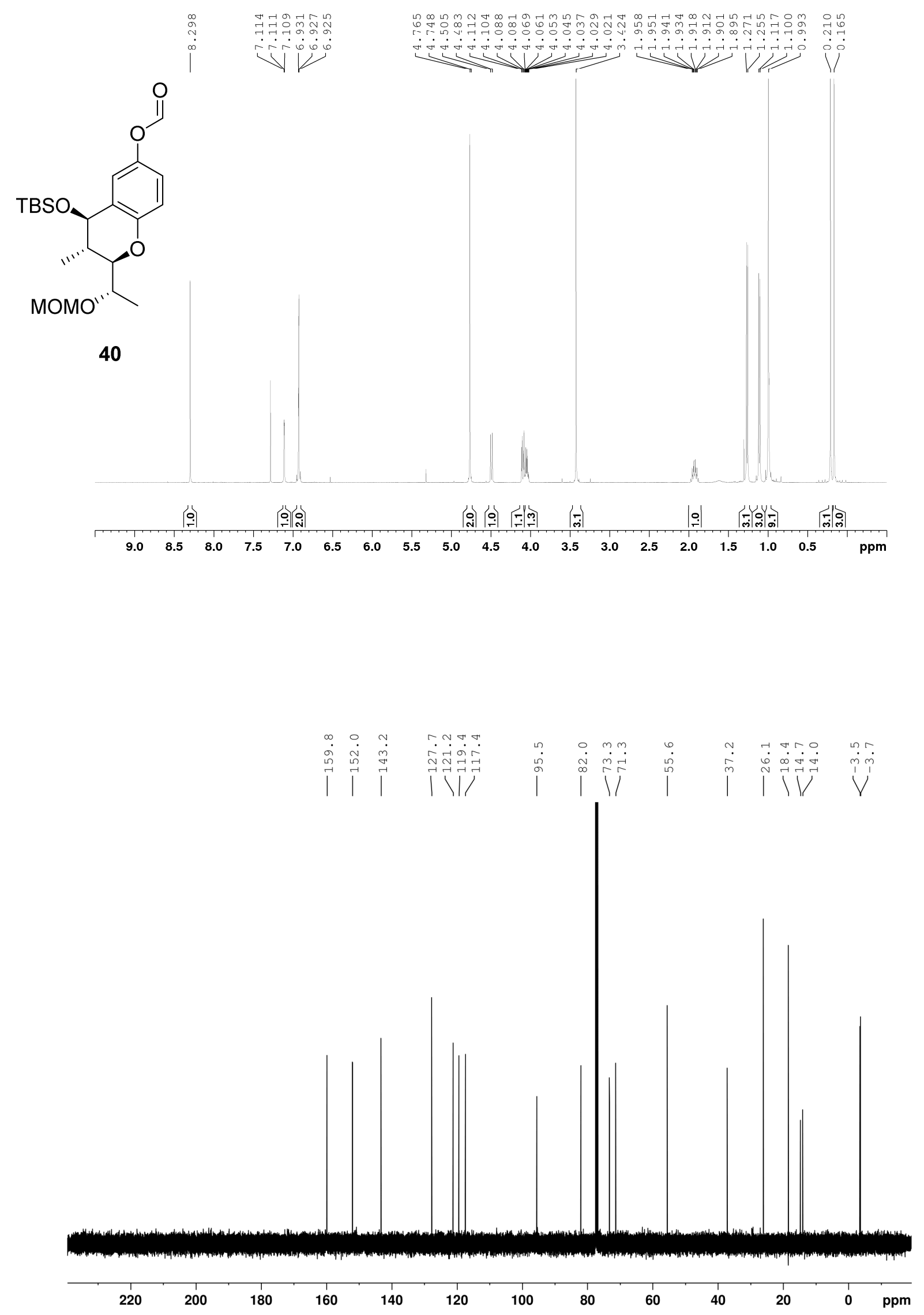

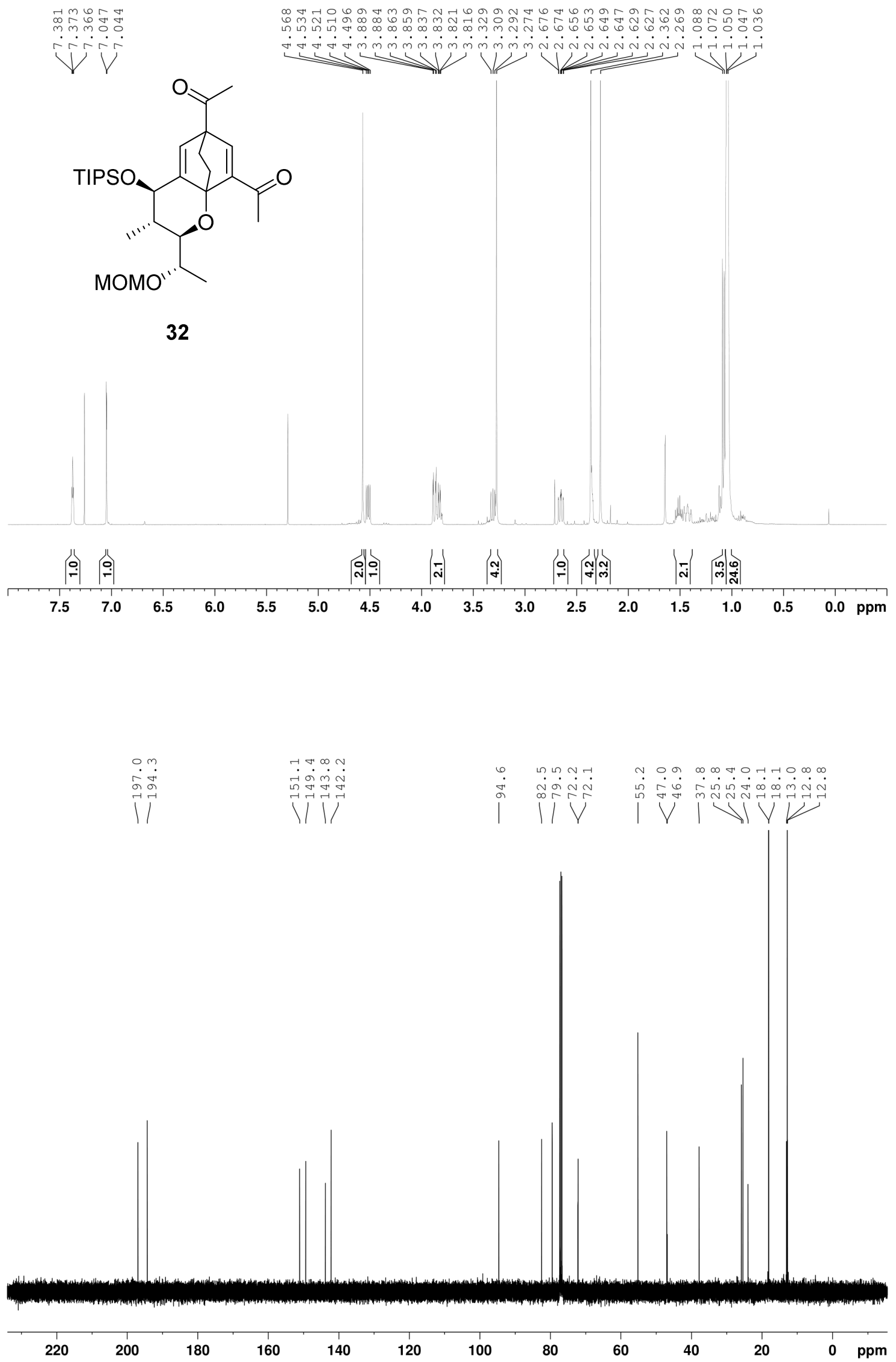


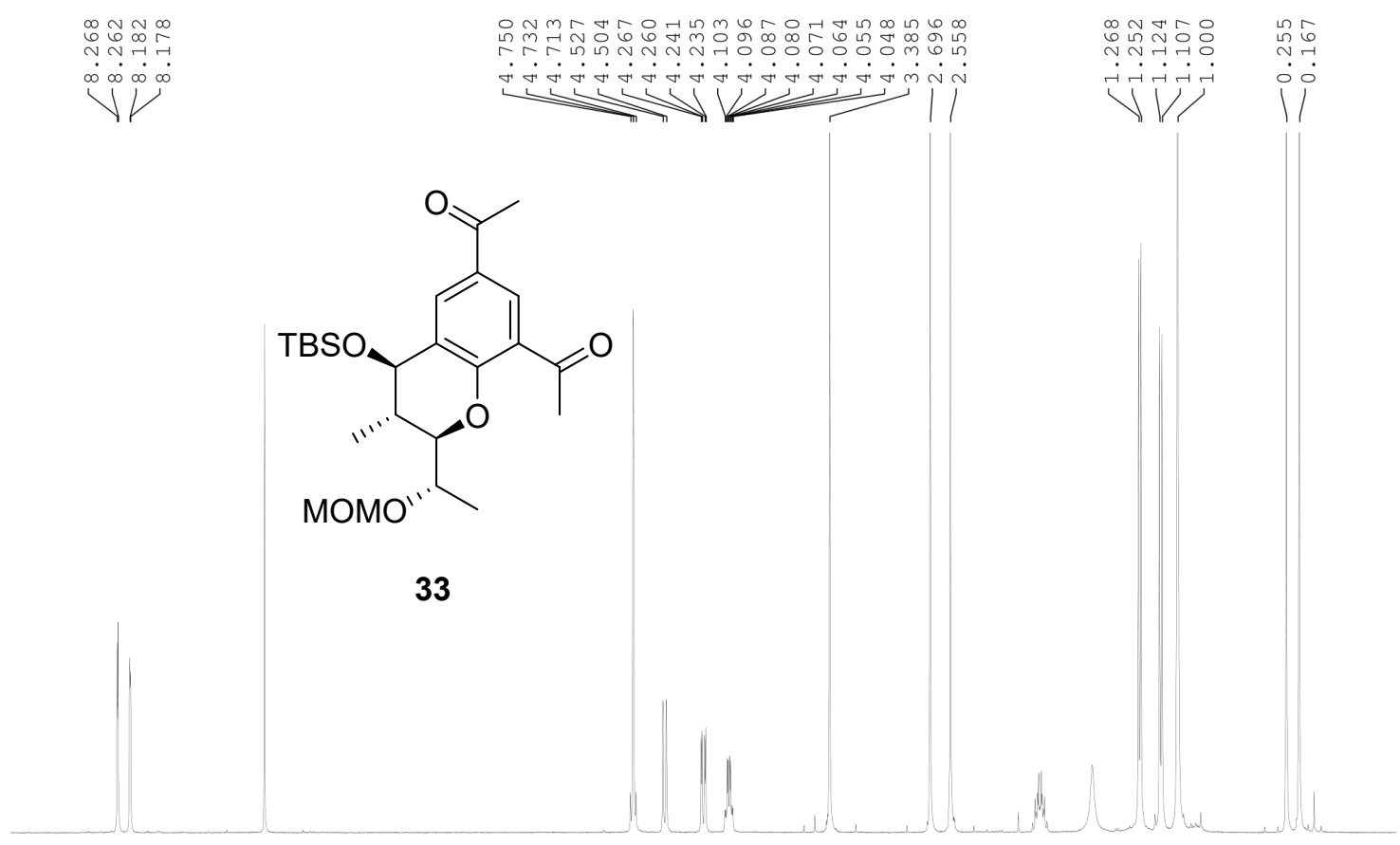

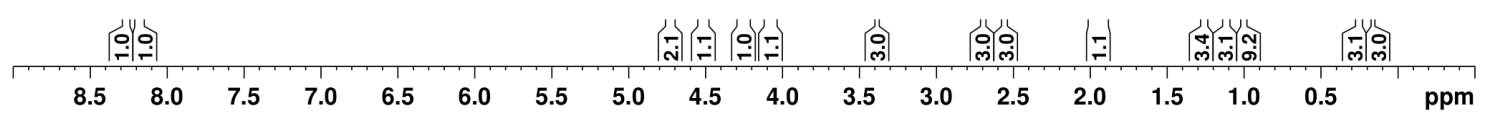

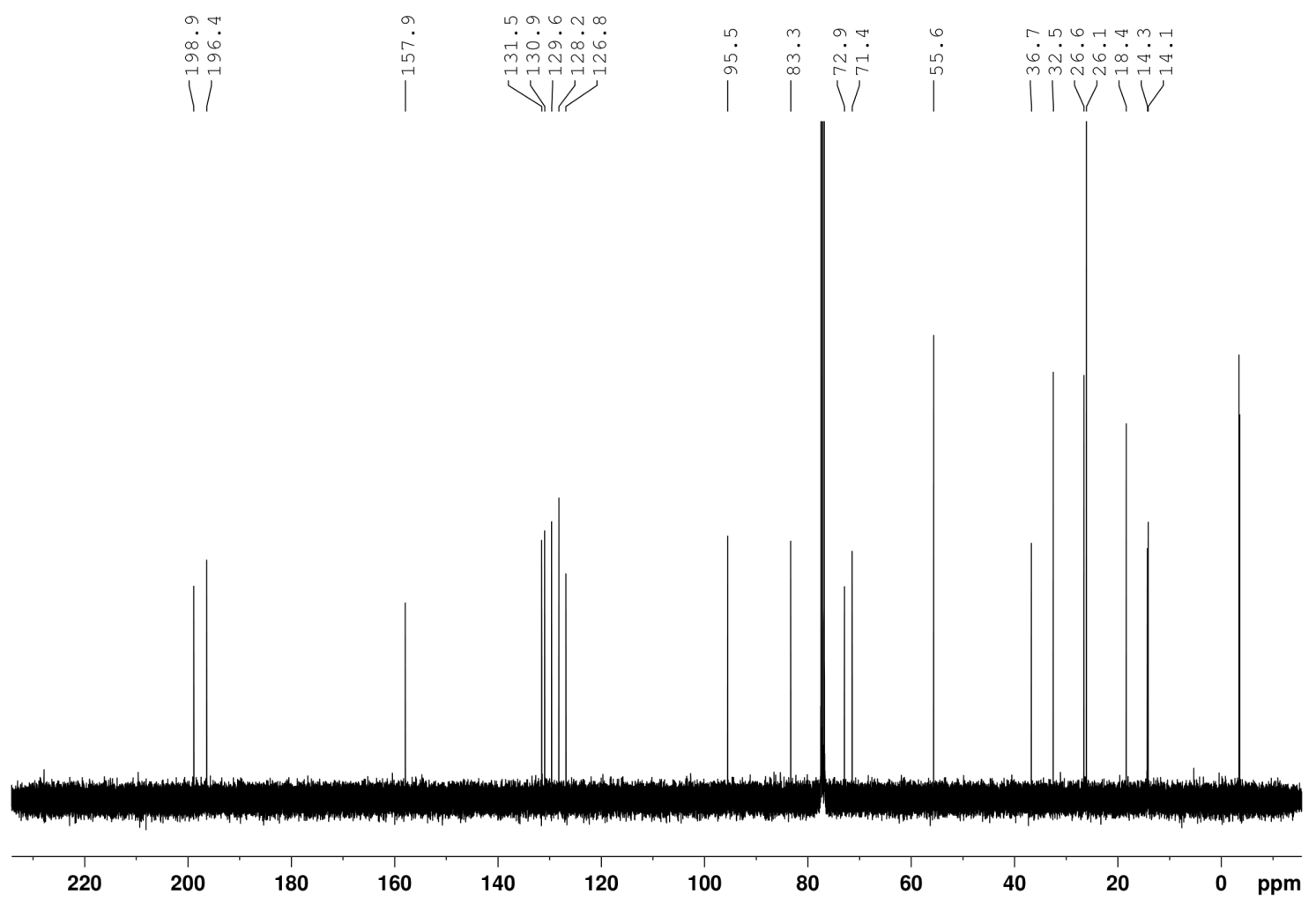

